\title{
Tunnel Effect for Kramers-Fokker-Planck Type Operators
}

\author{
Frédéric Hérau, Michael Hitrik, and Johannes Sjöstrand
}

\begin{abstract}
We consider operators of Kramers-Fokker-Planck type in the semiclassical limit such that the exponent of the associated Maxwellian is a Morse function with two local minima and a saddle point. Under suitable additional assumptions we establish the complete asymptotics of the exponentially small splitting between the first two eigenvalues.

Résumé. On considère des opérateurs du type de Kramers-Fokker-Planck dans la limite semi-classique tels que l'exposant du maxwellien associé soit une fonction de Morse avec deux minima et un point selle. Sous des hypothèses supplémentaires convenables on établit un développement asymptotique complet de l'écart exponentiellement petit entre les deux premières valeurs propres.
\end{abstract}

\section{Introduction}

This paper is a natural continuation of the work [14], investigating the low lying eigenvalues of the Kramers-Fokker-Planck operator

$$
P=y \cdot h \partial_{x}-V^{\prime}(x) \cdot h \partial_{y}+\frac{\gamma}{2}\left(-h \partial_{y}+y\right) \cdot\left(h \partial_{y}+y\right), \quad x, y \in \mathbf{R}^{n},
$$

where $\gamma>0$. Physically the semiclassical limit $h \rightarrow 0$ corresponds to the low temperature limit. As explained in [14], the original motivation for that work was to give more explicit versions of some results in [13] and later in [8], giving estimates on the time of return to equilibrium, or more or less equivalently, on the gap between the first eigenvalue 0 (when the potential $V$ tends to plus infinity sufficiently fast at infinity) and the second eigenvalue. See also [25] for further developments in that direction. The methods of those works as well as the one of Eckmann and Hairer [5] are inspired by those of hypoellipticity for Hörmander type operators. We will not repeat here all the motivations of [14], coming also 
from the works $[4,16]$ and others, including recent developments in pseudospectral theory.

In [14] it was assumed that $V$ in (1.1) is a smooth real Morse function with finitely many critical points, $U_{j}, j=1, \ldots, N$, that $\partial^{\alpha} V(x)$ is bounded for all multiindices $\alpha$ of length $|\alpha| \geq 2$ and that $|\nabla V(x)| \geq 1 / C$ for $|x| \geq C$ for some $C>0$. Under these assumptions it was shown that the eigenvalues in any disc $D(0, C h)$ are of the form

$$
\lambda_{j, k}(h) \sim h\left(\mu_{j, k}+h^{1 / N_{j, k}} \mu_{j, k, 1}+h^{2 / N_{j, k}} \mu_{j, k, 2}+\cdots\right), \quad h \rightarrow 0
$$

where the index $j$ labels the critical points and the leading coefficients $\mu_{j, k}$ can be given explicitly in terms of the Hessian of $V$ at the corresponding critical point. The values $\mu_{j, k}$ are confined to a sector $\left\{\left|\arg \left(z-\mu_{j, 0}\right)\right| \leq \theta_{j}\right\}$ for some $\theta_{j} \in\left[0, \frac{\pi}{2}\left[\right.\right.$, and $\lambda_{j, 0}$ is the eigenvalue with the smallest real part of all the $\lambda_{j, k}$. (This comes formally from a harmonic oscillator approximation.) Further $\mu_{j, k} \neq$ $\mu_{j, 0}$ for $k \neq 0$ and the asymptotic expansion (1.2) for $\lambda_{j, 0}$ contains only integer powers of $h$. See [14] for more details. Using this result, as well as control over the resolvent along suitable contours in the right half plane, the authors were able to give asymptotic expansions for large times of $\exp (-t P / h)$, that emphasize the role of the eigenvalues close to 0 given in (1.2). Indeed, there are no others in a certain parabolic neighborhood of the imaginary axis.

Moreover, we have $\mu_{j, 0} \geq 0$ with equality precisely when $U_{j}$ is a local minimum of the potential, and in the case of such a minimum it follows from the above results that $\lambda_{j, 0}$ is actually exponentially small.

In this paper we address the question of determining more precisely the size of these exponentially small eigenvalues. In the case when $V$ has precisely one local minimum, say $U_{1}$, and tends to $+\infty$, when $x \rightarrow \infty$, we know that the corresponding eigenvalue $\lambda_{1,0}$ is equal to zero (with the Maxwellian $\exp \left(-\left(y^{2} / 2+V(x)\right) / h\right)$ as the corresponding eigenfunction) and that this eigenvalue is separated from the other ones by a gap of size $h$. This means that we have return to equilibrium with a speed that is roughly 1 . The situation becomes more complicated when there is more than one local minimum. We are then in the presence of a tunneling problem which is much more complicated than the corresponding ones for the semiclassical Schrödinger operators since our operators are non-elliptic. In principle one should be able to follow the general approach of earlier works in the Schrödinger case as [9]. However it seems that one necessarily runs into a tunneling problem where the wave functions have to be studied also in a neighborhood of some intermediate saddle points of $V$, and as known from [10] that can indeed be done in the Schrödinger case with techniques that are very useful in a variety of problems. To carry out such an approach in the case of Kramers-Fokker-Planck would require one to accumulate the difficulties of non-resonant wells with the ones coming from the lack of ellipticity. This seems to lead to considerations of degenerate non-symmetric Finsler distances (see for instance $[1,18]$ ).

For the Witten Laplacian (see [11]) we are also in the presence of a tunneling problem with intermediate non-resonant wells and in that case one could avoid 
the detailed study near the non-resonant wells by studying directly the Witten complex as a tunneling problem between critical points of neighboring indices. More recently M. Klein, B. Helffer, and F. Nier [7] have used that approach to study the exponentially small non-vanishing eigenvalues of the Witten Laplacian. Also in [13], explicit estimates relating such small eigenvalues for the Witten Laplacian and the Kramers-Fokker-Planck operator were established.

This relation with the Witten complex was strengthened further in the works of J. Tailleur, S. Tanase-Nicola, J. Kurchan [24] and J. M. Bismut [2], who showed using respectively the languages of supersymmetry and differential forms, that the Kramers-Fokker-Planck operator can be viewed as a Witten Laplacian in degree 0 associated to a certain non-semidefinite scalar product in the spaces of differential forms. See also [17] for a quick introduction to the differential form version of Bismut and [3].

In the present paper, we use this supersymmetric approach. Our main result, valid also for a class of more general operators, is that if a certain weight function $\phi$ (which in the KFP-case is the function $y^{2} / 2+V(x)$ ) has precisely two local minima $U_{ \pm}$and an intermediate saddle point $U_{0}$ then we can get a complete asymptotic expansion for the second eigenvalue of the corresponding Witten Laplacian (reducing to the KFP operator in the special case). The logarithm of this eigenvalue is equal to $-2 h^{-1}\left(\min \left(\phi\left(U_{0}\right)-\phi\left(U_{1}\right), \phi\left(U_{0}\right)-\phi\left(U_{-1}\right)\right)+o(1)\right)$, but actually we do have a complete asymptotic expansion. See Theorem 11.1 for a complete statement. It seems clear that this result can be somewhat generalized but a more complete result might require exponential estimates and asymptotics for eigenfunctions also far from the critical points. In our present approach we are able to get such information for the eigenfunctions of the degree 0 operator in the basin of attraction of each minimum and for the degree 1 Laplacian in a small neighborhood of the saddle point.

In most of the paper we work with a scalar real second order non-elliptic operator, which is also non-selfadjoint, and we were led to reconsider some steps in [14]. The plan of the paper is the following:

In Section 2 we do some very simple and elementary exponential estimates mainly designed to get the appropriate control near infinity.

In Section 3 we establish the $m$-accretivity for our operators so that the step from a priori estimates to spectral information becomes possible.

In Section 4 we study certain auxiliary weights, somehow related to escape functions in resonance theory (see [12] and a large number of more recent works) in connection with some dynamical conditions.

In Section 5 we use those weights together with a machinery of Fourier integral operators with complex phase in order to get phase space a priori estimates away from the critical points. This section is perhaps technically the most complicated one, but the underlying ideas are now quite standard. Alternative methods are certainly possible and we might return to this step in future works. This section and the subsequent one are quite technical and should not be studied in detail in the first reading. 
In Section 6 we study the conjugation of our original operator under the Fourier integral operators of the preceding section and in Section 7 we finally obtain the a priori estimates that we need.

In Section 8 it is now quite easy to get detailed asymptotic results by adapting the methods of [14].

In Section 9 we show that the eigenfunction associated to $\lambda_{j, 0}$ for each (nondegenerate) critical point $U_{j}$ has the form $a(x ; h) e^{-\phi(x) / h}$ in a neighborhood of that point, where $a(x ; h)$ has an asymptotic expansion in integer powers of $h$ with coefficients in $C^{\infty}$ (neigh $\left.\left(U_{j}\right)\right)$ and $\phi=\phi_{j}$ is a smooth function of the order of magnitude $\left|x-U_{j}\right|^{2}$. It would be very interesting to extend such descriptions further away "beyond caustics".

In Section 10 we review the supersymmetric approach of $[2,24]$ (see also [17]) and establish various interesting links between the dynamical conditions of Section 4 and old results for non-selfadjoint operators with double characteristics [22]. This sheds additional light on some related computations in [14].

Finally in Section 11, we can put the various results together and establish the precise exponential asymptotics of the spectral gap between the first and the second eigenvalue (both real and the first one being zero.)

We expect that the spectral results of the present paper will give rise to precise asymptotics for the associated heat-evolution problem in the limit of large times and we plan to treat that problem in a separate paper.

\section{A priori estimates}

In this section we establish some simple a priori estimates which will be important in Section 6 and at other places. They illustrate the technique of gaining ellipticity by means of exponential weights that we shall later employ also in a micro-local setting.

Let $M$ denote either the space $\mathbf{R}^{n}$, or a smooth compact $n$-dimensional manifold equipped with a strictly positive smooth density of integration $d x$. On $M$ we consider a second order differential operator

$$
\begin{aligned}
P & =\sum_{j, k=1}^{n} h D_{x_{j}} \circ b_{j, k}(x) \circ h D_{x_{k}}+\frac{1}{2} \sum_{j=1}^{n}\left(c_{j}(x) h \partial_{x_{j}}+h \partial_{x_{j}} \circ c_{j}(x)\right)+p_{0}(x) \\
& =P_{2}+i P_{1}+P_{0}, \quad D_{x_{j}}=\frac{1}{i} \frac{\partial}{\partial x_{j}}
\end{aligned}
$$

where the coefficients $b_{j, k}, c_{j}, p_{0}$ are assumed to be smooth and real, with $b_{j, k}=$ $b_{k, j}$. In the manifold case, we use local coordinates such that $d x=d x_{1} \ldots d x_{n}$. To $P$ we associate the symbol in the semiclassical sense,

$$
\begin{aligned}
p(x, \xi) & =p_{2}(x, \xi)+i p_{1}(x, \xi)+p_{0}(x) \\
p_{2}(x, \xi) & =\sum_{j, k=1}^{n} b_{j, k}(x) \xi_{j} \xi_{k}, \quad p_{1}(x, \xi)=\sum_{j=1}^{n} c_{j}(x) \xi_{j},
\end{aligned}
$$


so that $p_{j}(x, \xi)$ is a real-valued polynomial in $\xi$, positively homogeneous of degree $j$. (It is well-defined on $T^{*} M$ and coincides with the Weyl symbol $\bmod \mathcal{O}\left(h^{2}\right)$ locally uniformly.) We assume that

$$
p_{2}(x, \xi) \geq 0, \quad p_{0}(x) \geq 0 .
$$

In the case $M=\mathbf{R}^{n}$, we impose the following growth conditions at infinity:

$$
\begin{aligned}
\partial_{x}^{\alpha} b_{j, k}(x) & =\mathcal{O}(1), & & |\alpha| \geq 0 \\
\partial_{x}^{\alpha} c_{j}(x) & =\mathcal{O}(1), & & |\alpha| \geq 1, \\
\partial_{x}^{\alpha} p_{0}(x) & =\mathcal{O}(1), & & |\alpha| \geq 2 .
\end{aligned}
$$

When discussing $P$ in the Operator theoretical sense we will assume that it is the closure of $P: \mathcal{S}(M) \rightarrow \mathcal{S}(M)$ as an unbounded Operator in $L^{2}(M)$. (When $M$ is compact, we identify the Schwartz space $\mathcal{S}(M)$ with $C^{\infty}(M)$.) Let $\mathcal{D}(P) \subset L^{2}(M)$ be the domain.

Lemma 2.1. We have

$$
\int p_{0}(x)|u(x)|^{2} d x+\int \sum_{j, k} b_{j, k}(x)\left(h D_{x_{j}} u\right)\left(\overline{h D_{x_{k}} u}\right) d x=\operatorname{Re}(P u \mid u),
$$

for all $u \in \mathcal{S}(M)$.

Proof. Immediate by integration by parts.

In the manifold case, we view $B(x)=\left(b_{j, k}(x)\right)$ as a positive semi-definite matrix $T_{x}^{*} M \rightarrow T_{x} M$ and if we choose some smooth Riemannian metric on $M$, we can view $B(x)$ as a map $T_{x}^{*} M \rightarrow T_{x}^{*} M$ and define $B(x)^{\frac{1}{2}}$ similarly. (2.8) then becomes

$$
\left\|p_{0}(x)^{\frac{1}{2}} u\right\|^{2}+\left\|B(x)^{\frac{1}{2}} h D u\right\|^{2}=\operatorname{Re}(P u \mid u),
$$

implying

$$
\left\|p_{0}^{\frac{1}{2}} u\right\|+\left\|B^{\frac{1}{2}} h D u\right\| \leq C_{0}(\|P u\|+\|u\|) .
$$

In particular, $u \in \mathcal{D}(P) \Rightarrow\left\|p_{0}^{\frac{1}{2}} u\right\|+\left\|B^{\frac{1}{2}} h D u\right\|<\infty$.

Using the anti-selfadjoint part $i P_{1}$ we shall obtain a similar estimate where the averages of $p_{0}$ along the trajectories of

$$
\nu\left(x, \partial_{x}\right)=\sum_{1}^{n} c_{j}(x) \partial_{x_{j}}
$$

will play a role.

In general, if $\psi(x)$ is a smooth real-valued function, the Operator

$$
P_{\psi}:=e^{\psi / h} \circ P \circ e^{-\psi / h}
$$


is of the same form as (2.1) with new coefficients $c_{j}, p_{0}$ and the new symbol

$$
\begin{aligned}
p_{\psi}(x, \xi)= & p_{2}\left(x, \xi+i \psi^{\prime}(x)\right)+i p_{1}\left(x, \xi+i \psi^{\prime}(x)\right)+p_{0}(x) \\
= & p_{2}(x, \xi)+i\left(p_{1}(x, \xi)+\partial_{\xi} p_{2}\left(x, \psi^{\prime}(x)\right) \cdot \xi\right) \\
& +p_{0}(x)-p_{1}\left(x, \psi^{\prime}(x)\right)-p_{2}\left(x, \psi^{\prime}(x)\right) \\
= & : p_{2}(x, \xi)+i p_{1, \psi}(x, \xi)+p_{0, \psi}(x)
\end{aligned}
$$

In this section we choose $\psi$ very small and treat $p_{2}\left(x, \psi^{\prime}(x)\right)$ as a perturbation. Notice that

$$
p_{1}\left(x, \psi^{\prime}(x)\right)=\nu\left(x, \partial_{x}\right) \psi .
$$

Let $f(t) \in C^{\infty}([0, \infty[;[0,3 / 2])$ be an increasing function with $f(t)=t$ on $[0,1], f(t)=3 / 2$ on $\left[2, \infty\left[, f(t) \leq t\right.\right.$. Put $f_{\epsilon}(t)=\epsilon f(t / \epsilon)$ and consider for $T_{0}>0$ fixed,

$$
\psi_{\epsilon}=\int k\left(\frac{t}{T_{0}}\right) f_{\epsilon} \circ p_{0} \circ \exp (t \nu) d t
$$

where

$$
k(t)=\left\{\begin{array}{l}
0, \quad|t| \geq 1 / 2 \\
t+\frac{1}{2}, \quad-\frac{1}{2} \leq t<0 \\
-k(-t), \quad 0<t \leq \frac{1}{2}
\end{array}\right.
$$

Then

$$
\nu\left(\psi_{\epsilon}\right)=f_{\epsilon} \circ p_{0}-\left\langle f_{\epsilon} \circ p_{0}\right\rangle_{T_{0}}
$$

where

$$
\left\langle f_{\epsilon} \circ p_{0}\right\rangle_{T_{0}}=\frac{1}{T_{0}} \int_{-T_{0} / 2}^{T_{0} / 2} f_{\epsilon} \circ p_{0} \circ \exp (t \nu) d t
$$

is the time $T_{0}$ average of $f_{\epsilon} \circ p_{0}$ along the integral curves of $\nu$. Clearly,

$$
\left|\psi_{\epsilon}\right| \leq \frac{T_{0}}{4} \frac{3}{2} \epsilon=\frac{3 T_{0}}{8} \epsilon .
$$

From (2.6) it is easy to see that $\Phi_{t}(x):=\exp (t \nu)(x)$ is well-defined for all $t \in \mathbf{R}, x \in M$ and that

$$
\left|\partial_{x}^{\alpha} \Phi_{t}(x)\right| \leq C(\alpha) e^{C_{0}|\alpha||t|}, \quad \alpha \in \mathbf{N}^{n}, \quad|\alpha| \geq 1 .
$$

In particular, $\left|\partial_{x}^{\alpha} \Phi_{t}(x)\right| \leq C_{\alpha, T_{0}},|t| \leq T_{0}$. On the other hand, since $p_{0} \geq 0$, $p_{0}^{\prime \prime}=\mathcal{O}(1)$ (by $\left.(2.7)\right)$, we know that $\left|p_{0}^{\prime}\right| \leq \mathcal{O}(1) p_{0}^{\frac{1}{2}}$ and this quantity is $\mathcal{O}\left(\epsilon^{1 / 2}\right)$ in the region where $0 \leq p_{0} \leq 2 \epsilon$. It follows that $\partial^{\alpha}\left(f_{\epsilon} \circ p_{0}\right)=\mathcal{O}\left(\epsilon^{1-|\alpha| / 2}\right), \alpha \in \mathbf{N}^{n}$ and together with (2.19), we get

$$
\partial_{x}^{\alpha} \psi_{\epsilon}=\mathcal{O}\left(\epsilon^{1-|\alpha| / 2}\right), \quad \forall \alpha \in \mathbf{N}^{n},
$$

for every fixed $T_{0}$. 
Then from (2.13), (2.14), (2.16), (2.20) and the uniform boundedness of the $b_{j, k}$, we get

$$
\begin{aligned}
p_{0, \delta \psi_{\epsilon}} & =p_{0}(x)-\delta f_{\epsilon} \circ p_{0}+\delta\left\langle f_{\epsilon} \circ p_{0}\right\rangle_{T_{0}}-\delta^{2} p_{2}\left(x, \psi_{\epsilon}^{\prime}\right) \\
& =p_{0}(x)-\delta f_{\epsilon} \circ p_{0}+\delta\left\langle f_{\epsilon} \circ p_{0}\right\rangle_{T_{0}}-\mathcal{O}\left(\delta^{2} \epsilon\right),
\end{aligned}
$$

uniformly on $\mathbf{R}^{n}$ for $0 \leq \delta \leq 1$. Using the properties of $f$, we notice that $p_{0}(x)-$ $\delta f_{\epsilon} \circ p_{0} \geq(1-\delta) p_{0}$, so

$$
p_{0, \delta \psi_{\epsilon}} \geq(1-\delta) p_{0}+\delta\left\langle f_{\epsilon} \circ p_{0}\right\rangle_{T_{0}}-\mathcal{O}\left(\delta^{2} \epsilon\right) .
$$

Since the coefficients of $P_{\delta \psi_{\epsilon}}$ grow at most polynomially, Lemma 2.1 can be applied and gives

$$
\int\left(p_{0, \delta \psi_{\epsilon}}(x)-\operatorname{Re} z\right)|u|^{2} d x \leq \operatorname{Re}\left(\left(P_{\delta \psi_{\epsilon}}-z\right) u \mid u\right), \quad z \in \mathbf{C}, \quad \operatorname{Re} z \leq \frac{\epsilon}{Z},
$$

where $Z \gg 1$ is independent of $\epsilon$. (For Lemma 2.1 we do not need that $p_{0} \geq 0$.) Let $\mu>0$ and rewrite $(2.23)$ as

$$
\begin{aligned}
\int \max \left(\widetilde{p}_{0, \psi}, \mu\right)|u|^{2} d x \leq & \operatorname{Re}\left(\left(\left(P_{\psi}-z\right)+\left(\mu-\widetilde{p}_{0, \psi}\right)_{+}\right) u \mid u\right) \\
= & \operatorname{Re}\left(\operatorname { m a x } ( \widetilde { p } _ { 0 , \psi } , \mu ) ^ { - \frac { 1 } { 2 } } \left(P_{\psi}-z\right.\right. \\
& \left.\left.+\left(\mu-\widetilde{p}_{0, \psi}\right)_{+}\right) u \mid \max \left(\widetilde{p}_{0, \psi}, \mu\right)^{\frac{1}{2}} u\right) .
\end{aligned}
$$

Here we write $\psi$ for $\delta \psi_{\epsilon}$ and $\widetilde{p}_{0, \psi}=p_{0, \psi}-\operatorname{Re} z$.

Then using Cauchy-Schwarz,

$\left\|\max \left(\widetilde{p}_{0, \psi}, \mu\right)^{\frac{1}{2}} u\right\| \leq\left\|\max \left(\widetilde{p}_{0, \psi}, \mu\right)^{-\frac{1}{2}}\left(P_{\psi}-z\right) u\right\|+\left\|\frac{\left(\mu-\widetilde{p}_{0, \psi}\right)_{+}}{\mu} \max \left(\widetilde{p}_{0, \psi}, \mu\right)^{\frac{1}{2}} u\right\|$, leading to

$$
\begin{aligned}
\left\|\max \left(\widetilde{p}_{0, \psi}, \mu\right)^{\frac{1}{2}} u\right\| \leq & \left\|\max \left(\widetilde{p}_{0, \psi}, \mu\right)^{-\frac{1}{2}}\left(P_{\psi}-z\right) u\right\| \\
& +\left\|1_{\left\{\widetilde{p}_{0, \psi} \leq \mu\right\}} \max \left(\widetilde{p}_{0, \psi}, \mu\right)^{\frac{1}{2}} u\right\| .
\end{aligned}
$$

Notice that $1_{\left\{\widetilde{p}_{0, \psi} \leq \mu\right\}} \max \left(\widetilde{p}_{0, \psi}, \mu\right)^{\frac{1}{2}}=1_{\left\{\widetilde{p}_{0, \psi} \leq \mu\right\}} \mu^{\frac{1}{2}}$.

We have

$$
\begin{aligned}
\widetilde{p}_{0, \psi} & =p_{0}(x)-\delta f_{\epsilon} \circ p_{0}+\delta\left\langle f_{\epsilon} \circ p\right\rangle_{T_{0}}-\mathcal{O}\left(\delta^{2} \epsilon\right)-\operatorname{Re} z \\
& \geq p_{0}(x)-\delta f_{\epsilon} \circ p_{0}+\delta\left\langle f_{\epsilon} \circ p\right\rangle_{T_{0}}-\mathcal{O}\left(\delta^{2} \epsilon\right)-\frac{\epsilon}{Z} .
\end{aligned}
$$

Choose $Z=\delta^{-2}$, so that

$$
\widetilde{p}_{0, \psi} \geq p_{0}(x)-\delta f_{\epsilon} \circ p_{0}+\delta\left\langle f_{\epsilon} \circ p\right\rangle_{T_{0}}-\mathcal{O}\left(\delta^{2} \epsilon\right) .
$$

Choose $\delta>0$ small enough (but independent of $\epsilon$ ) so that $\mathcal{O}\left(\delta^{2} \epsilon\right) \leq \delta \epsilon / C_{0}$, where we shall fix $C_{0}$ sufficiently large. Then

$$
\widetilde{p}_{0, \psi} \geq(1-\delta) p_{0}(x)+\delta\left(\left\langle f_{\epsilon} \circ p_{0}\right\rangle_{T_{0}}-\frac{\epsilon}{C_{0}}\right) .
$$


When $\left\langle f_{\epsilon} \circ p_{0}\right\rangle_{T_{0}} \geq \frac{2 \epsilon}{3}$, we get

$$
\widetilde{p}_{0, \psi} \geq(1-\delta) p_{0}(x)+\frac{\delta}{2} .
$$

When $\left\langle f_{\epsilon} \circ p_{0}\right\rangle_{T_{0}}<\frac{2 \epsilon}{3}$ we have $\widetilde{p}_{0, \psi} \geq(1-\delta) p_{0}-\frac{\delta \epsilon}{C_{0}}$.

Choose

$$
\mu=\frac{\delta \epsilon}{C_{0}} .
$$

Then

$$
(1-\delta) p_{0}+\mu \leq \widetilde{p}_{0, \psi}+2 \mu \leq 3 \max \left(\widetilde{p}_{0, \psi}, \mu\right) .
$$

Moreover, if $\widetilde{p}_{0, \psi} \leq \mu$, then $\delta\left\langle f_{\epsilon} \circ p_{0}\right\rangle_{T_{0}}-\frac{\delta \epsilon}{C_{0}} \leq \frac{\delta \epsilon}{C_{0}}$, so $\left\langle f_{\epsilon} \circ p_{0}\right\rangle_{T_{0}} \leq \frac{2 \epsilon}{C_{0}}$. From (2.24) and (2.25) we then infer that

$$
\begin{aligned}
\left\|\left((1-\delta) p_{0}+\frac{\delta \epsilon}{C_{0}}\right)^{\frac{1}{2}} u\right\| \leq & 3\left\|\left((1-\delta) p_{0}+\frac{\delta \epsilon}{C_{0}}\right)^{-\frac{1}{2}}\left(P_{\psi}-z\right) u\right\| \\
& +\sqrt{3} \sqrt{\frac{\delta \epsilon}{C_{0}}}\|u\|_{\left\{\left\langle f_{\epsilon} \circ p_{0}\right\rangle_{T_{0}} \leq \frac{2 \epsilon}{C_{0}}\right\}},
\end{aligned}
$$

for $\operatorname{Re} z \leq \frac{\epsilon}{Z}$.

Here we can take $\epsilon=M h$ with $M \gg 1$. Then by (2.18) we have

$$
|\psi / h|=\left|\delta \psi_{\epsilon} / h\right| \leq C\left(M, T_{0}\right) \delta,
$$

so there is a constant $C$ independent of $h$ (but depending on $M, \delta$ ) such that

$$
\frac{1}{C} \leq e^{\psi / h} \leq C
$$

From the discussion above, in particular (2.26), we get

Proposition 2.2. Let $P$ be of the form (2.1), where $b_{j, k}, c_{j}, p_{0}$ are smooth and real and satisfy (2.2)-(2.7). Define $\left\langle f_{\epsilon} \circ p_{0}\right\rangle_{T_{0}}$ as in (2.17) with $f_{\epsilon}$ defined after (2.14). Then for every $C>0$, there exists $\widetilde{C}>0$ such that

$$
\left\|\left(p_{0}+h\right)^{\frac{1}{2}} u\right\| \leq \widetilde{C}\left(\left\|\left(p_{0}+h\right)^{-\frac{1}{2}}(P-z) u\right\|+h^{\frac{1}{2}}\|u\|_{\left\{\left\langle f_{\widetilde{C} \epsilon} \circ p_{0}\right\rangle_{T_{0}} \leq \widetilde{C} h\right\}}\right),
$$

for $u \in \mathcal{S}$, Re $z \leq C h$.

Notice that (2.8) implies that

$$
\left\|B^{\frac{1}{2}} h D u\right\|^{2} \leq\left\|\left(p_{0}+h\right)^{-\frac{1}{2}}(P-z) u\right\|\left\|\left(p_{0}+h\right)^{\frac{1}{2}} u\right\|+C\left\|h^{\frac{1}{2}} u\right\|^{2} .
$$

\section{From injectivity to the resolvent}

Let $P=P_{2}+i P_{1}+P_{0}$ with symbol $p=p_{2}+i p_{1}+p_{0}$ be as in Section 2 , so that we have $(2.8)$

$$
\int p_{0}(x)|u(x)|^{2} d x+\left(P_{2} u \mid u\right)=\operatorname{Re}(P u \mid u), \quad u \in \mathcal{S}(M),
$$


leading to

$$
|\operatorname{Re} z|\|u\|^{2}+\left\|p_{0}^{\frac{1}{2}} u\right\|^{2}+\left(P_{2} u \mid u\right)=\operatorname{Re}((P-z) u \mid u) \leq\|(P-z) u\|\|u\|, \quad \operatorname{Re} z<0 .
$$

We get

$$
\begin{aligned}
\left\|\left(|\operatorname{Re} z|+p_{0}\right)^{\frac{1}{2}} u\right\|^{2} & \leq\left\|\left(|\operatorname{Re} z|+p_{0}\right)^{-\frac{1}{2}}(P-z) u\right\|\left\|\left(|\operatorname{Re} z|+p_{0}\right)^{\frac{1}{2}} u\right\|, \\
\left\|\left(|\operatorname{Re} z|+p_{0}\right)^{\frac{1}{2}} u\right\| & \leq\left\|\left(|\operatorname{Re} z|+p_{0}\right)^{-\frac{1}{2}}(P-z) u\right\|, \\
|\operatorname{Re} z|^{\frac{1}{2}}\left\|\left(|\operatorname{Re} z|+p_{0}\right)^{\frac{1}{2}} u\right\| & \leq\|(P-z) u\|, \\
|\operatorname{Re} z|\|u\| & \leq\|(P-z) u\| .
\end{aligned}
$$

From this we get

$$
\left(P_{2} u \mid u\right) \leq\|(P-z) u\|\|u\| \leq \frac{1}{|\operatorname{Re} z|}\|(P-z) u\|^{2},
$$

and putting some of the estimates together,

$$
|\operatorname{Re} z|^{2}\|u\|^{2}+|\operatorname{Re} z|\left\|p_{0}^{\frac{1}{2}} u\right\|^{2}+|\operatorname{Re} z|\left(P_{2} u \mid u\right) \leq 2\|(P-z) u\|^{2} .
$$

By $P$ we also denote the graph closure of $P: \mathcal{S} \rightarrow \mathcal{S}$. From the estimates above we see that the range $\mathcal{R}(P-z)$ is closed in $L^{2}$ when $\operatorname{Re} z<0$.

\section{Proposition 3.1.}

$$
\mathcal{R}(P-z)=L^{2}, \quad \text { Re } z<0 .
$$

Proof. It suffices to prove that $\mathcal{R}(P-z)=L^{2}$ for some $z$ with $\operatorname{Re} z<0$, because the a priori estimate then implies that $\left\|(P-z)^{-1}\right\| \leq|\operatorname{Re} z|^{-1}$ and this fact extends by standard arguments to the whole left half plane. For the same $z$ it suffices to show that if $u \in L^{2}$ and $\left(P^{*}-\bar{z}\right) u=0$ in the sense of distributions, then $u=0$. Now the formal adjoint $P^{*}=P_{2}-i P_{1}+P_{0}$ has the same properties as $P$, so in order to simplify the notations, we may just as well prove the corresponding fact for $P-z$ instead of $P^{*}-\bar{z}$ : There exists a $z$ with $\operatorname{Re} z<0$ such that if $u \in L^{2}$ and $(P-z) u=0$ in the sense of distributions, then $u=0$.

When $M=\mathbf{R}^{n}$, let $\mathrm{Op}_{h}(q)$ denote the Weyl quantization of $q(x, h \xi)$ and put

$$
\Lambda_{\epsilon}=\mathrm{Op}_{h}\left(\langle\epsilon(x, \xi)\rangle^{-N}\right)
$$

where $N \geq 2$ is fixed and $\epsilon>0$ is small and fixed. (When $M$ is a compact manifold, choose a Riemannian metric and put $\Lambda_{\epsilon}=\left(1-\epsilon h^{2} \Delta\right)^{-N / 2}$.) Consider the equation

$$
(P-z) u=v, \quad u, v \in L^{2} .
$$

Then $\Lambda_{\epsilon}(P-z) u=\Lambda_{\epsilon} v$, so

$$
(P-z) \Lambda_{\epsilon} u=\Lambda_{\epsilon} v+\left[P, \Lambda_{\epsilon}\right] u .
$$

Since $N \geq 2$, we can find a sequence $u_{j} \in \mathcal{S}$ such that $\Lambda_{\epsilon} u_{j} \rightarrow \Lambda_{\epsilon} u, p_{0}^{\frac{1}{2}} \Lambda_{\epsilon} u_{j} \rightarrow$ $p_{0}^{\frac{1}{2}} \Lambda_{\epsilon} u, \Lambda_{\epsilon}(P-z) u_{j} \rightarrow \Lambda_{\epsilon} v,\left[P, \Lambda_{\epsilon}\right] u_{j} \rightarrow\left[P, \Lambda_{\epsilon}\right] u$ in $L^{2},\left(P_{2} \Lambda_{\epsilon} u_{j} \mid \Lambda_{\epsilon} u_{j}\right) \rightarrow$ 
$\left(P_{2} \Lambda_{\epsilon} u \mid \Lambda_{\epsilon} u\right)$. This means that (3.1) is applicable to (3.2), and we get

$$
\begin{array}{r}
|\operatorname{Re} z|^{2}\left\|\Lambda_{\epsilon} u\right\|^{2}+|\operatorname{Re} z|\left\|p_{0}^{\frac{1}{2}} \Lambda_{\epsilon} u\right\|^{2}+|\operatorname{Re} z|\left(P_{2} \Lambda_{\epsilon} u \mid \Lambda_{\epsilon} u\right) \\
\leq 4\left(\left\|\Lambda_{\epsilon} v\right\|^{2}+\left\|\left[P, \Lambda_{\epsilon}\right] u\right\|^{2}\right) .
\end{array}
$$

Consider $\left[P, \Lambda_{\epsilon}\right] u=\left(\left[P, \Lambda_{\epsilon}\right] \Lambda_{\epsilon}^{-1}\right) \Lambda_{\epsilon} u$. We study the Operator $\left[P, \Lambda_{\epsilon}\right] \Lambda_{\epsilon}^{-1}$ and assume that $M=\mathbf{R}^{n}$ in order to fix the ideas. If $\rho=(x, \xi)$, we have

$$
\partial_{\rho}^{\alpha}\langle\rho\rangle^{-N}=\mathcal{O}(1)\langle\rho\rangle^{-N-|\alpha|},
$$

so

$$
\partial_{\rho}^{\alpha}\langle\epsilon \rho\rangle^{-N}=\mathcal{O}(1)\langle\epsilon \rho\rangle^{-N}\left(\frac{\epsilon}{\langle\epsilon \rho\rangle}\right)^{|\alpha|}=\mathcal{O}(1)\langle\epsilon \rho\rangle^{-N}\langle\rho\rangle^{-|\alpha|},
$$

uniformly with respect to $\epsilon$. From this we deduce that the symbol of $\left[P, \Lambda_{\epsilon}\right]$ is $\frac{h}{i}\left\{p, \Lambda_{\epsilon}\right\}+\mathcal{O}_{0}\left(h^{2} \Lambda_{\epsilon}\right)$, using the same letters for Operators and their symbols (except for $P, P_{j}$ where we already introduced a distinction by using lower case letters for the symbols), and using the notation $\mathcal{O}_{0}\left(h^{2} \Lambda_{\epsilon}\right)$ for a symbol $q$ satisfying $\partial_{\rho}^{\alpha} q=\mathcal{O}_{\alpha}\left(h^{2} \Lambda_{\epsilon}\right)$, uniformly in $\epsilon$. Here

$$
\left\{p, \Lambda_{\epsilon}\right\}=\mathcal{O}_{0}\left(\Lambda_{\epsilon}(\rho)\langle\rho\rangle\right),
$$

so the symbol of $\left[\Lambda_{\epsilon}, P\right] \Lambda_{\epsilon}^{-1}$ is

$$
\frac{h}{i} \frac{\left\{p, \Lambda_{\epsilon}\right\}}{\Lambda_{\epsilon}}+\mathcal{O}_{0}\left(h^{2}\right) .
$$

Recall that $p(x, \xi)=p_{0}(x)+i p_{1}(x, \xi)+p_{2}(x, \xi)$. We get

$$
\begin{aligned}
\frac{\left\{p_{0}, \Lambda_{\epsilon}\right\}}{\Lambda_{\epsilon}} & =\mathcal{O}_{0}\left(\frac{\langle x\rangle}{\langle\rho\rangle}\right)=\mathcal{O}_{0}(1), \\
\frac{\left\{p_{1}, \Lambda_{\epsilon}\right\}}{\Lambda_{\epsilon}} & =\mathcal{O}_{0}\left(\frac{\langle x\rangle}{\langle\rho\rangle}\right)+\mathcal{O}_{0}\left(\frac{\langle\xi\rangle}{\langle\rho\rangle}\right)=\mathcal{O}_{0}(1), \\
\frac{\left\{p_{2}, \Lambda_{\epsilon}\right\}}{\Lambda_{\epsilon}} & =Q+\mathcal{O}_{0}(1), \\
Q & =-\frac{\nabla_{\xi} \Lambda_{\epsilon}}{\Lambda_{\epsilon}} \cdot \nabla_{x} p_{2}=\mathcal{O}_{0}\left(\frac{1}{\langle\rho\rangle}\right) \cdot \nabla_{x} p_{2}(\rho) .
\end{aligned}
$$

From these computations, we retain that

$$
\left\|\left[P, \Lambda_{\epsilon}\right] \Lambda_{\epsilon}^{-1} w\right\| \leq \mathcal{O}(h)\|w\|+\mathcal{O}(h)\|Q w\| .
$$

The symbol $Q$ is real and $\partial_{\xi}^{\alpha} Q=\mathcal{O}_{0}\left(\langle\xi\rangle^{2-|\alpha|} /\langle\rho\rangle\right)$, so

$$
\|Q w\|^{2}=\left(Q^{2} w \mid w\right)
$$

where $Q \circ Q$ has the symbol $Q^{2}+\mathcal{O}_{0}\left(h^{2}\right)$.

Since $p_{2} \geq 0, \partial_{x}^{2} p_{2}=\mathcal{O}\left(\xi^{2}\right)$ we know that

$$
\left|\partial_{x} p_{2}\right| \leq \mathcal{O}(1)|\xi| p_{2}(x, \xi)^{\frac{1}{2}},
$$


and we conclude that on the symbol level

$$
Q^{2} \leq C p_{2} .
$$

Hence by the semi-classical Fefferman-Phong inequality for operators with symbols in the Hörmander class $S_{1,0}^{2}$ (see for example Subsection 7.2 in [14] for a short review of the Weyl-Hörmander calculus):

$$
\|Q w\|^{2} \leq C\left(P_{2} w \mid w\right)+\mathcal{O}\left(h^{2}\right)\|w\|^{2} .
$$

Using this in (3.4), we get

$$
\left\|\left[P, \Lambda_{\epsilon}\right] \Lambda_{\epsilon}^{-1} w\right\|^{2} \leq C h^{2}\left(\left(P_{2} w \mid w\right)+\|w\|^{2}\right) .
$$

we use this in (3.3) with $\left[P, \Lambda_{\epsilon}\right] u=\left[P, \Lambda_{\epsilon}\right] \Lambda_{\epsilon}^{-1} \Lambda_{\epsilon} u$ and get

$$
\begin{array}{r}
|\operatorname{Re} z|\left\|p_{0}^{\frac{1}{2}} \Lambda_{\epsilon} u\right\|^{2}+\left(|\operatorname{Re} z|^{2}-C h^{2}\right)\left\|\Lambda_{\epsilon} u\right\|^{2}+\left(|\operatorname{Re} z|-C h^{2}\right)\left(P_{2} \Lambda_{\epsilon} u \mid \Lambda_{\epsilon} u\right) \\
\leq 4\left\|\Lambda_{\epsilon}(P-z) u\right\|^{2} .
\end{array}
$$

So if $\operatorname{Re} z<-\sqrt{C} h$ and $(P-z) u=0, u \in L^{2}$, we have $u=0$.

Corollary 3.2. The maximal closed extension $P_{\max }$ of $P$ (with domain given by $\{u \in$ $\left.L^{2} ; P u \in L^{2}\right\}$ ) coincides with the graph closure (the minimal closed extension), already introduced.

Proof. Let $z$ be fixed with Re $z<-\sqrt{C} h$. Let $u, v \in L^{2}$ with $\left(P_{\max }-z\right) u=v$. Denote by $P_{\min }$ the graph closure. Since $\mathcal{R}\left(P_{\min }-z\right)=L^{2}$, there exists $\widetilde{u} \in \mathcal{D}\left(P_{\min }\right)$ such that $\left(P_{\min }-z\right) \widetilde{u}=v$. Hence $(P-z)(u-\widetilde{u})=0$ in the sense of distributions. We saw in the proof of the proposition that this implies that $u-\widetilde{u}=0$.

This result can be extended to the following auxiliary problem: Let $R_{-}$: $\mathbf{C}^{N} \rightarrow L^{2}, R_{+}: L^{2} \rightarrow \mathbf{C}^{N}$ be bounded Operators and assume that

$$
\mathcal{P}(z)=\left(\begin{array}{cc}
P-z & R_{-} \\
R_{+} & 0
\end{array}\right): \mathcal{D}(P) \times \mathbf{C}^{N} \rightarrow L^{2} \times \mathbf{C}^{N},
$$

is injective with

$$
\|u\|+\left|u_{-}\right| \leq C(h)\left(\|v\|+\left|v_{+}\right|\right), \quad z \in \Omega(h),
$$

whenever

$$
\mathcal{P}(z)\left(\begin{array}{c}
u \\
u_{-}
\end{array}\right)=\left(\begin{array}{c}
v \\
v_{+}
\end{array}\right) .
$$

Here we assume that $\Omega(h)$ is open and connected, intersecting the resolvent set $\rho(P)$ of $P$.

From (3.7), we see that $\mathcal{P}(z)$ is injective and has closed range for every $z \in \Omega(h)$. When $z$ is also in the resolvent set of $P$, we can rewrite (3.8) as

$$
\left(\begin{array}{cc}
1 & (P-z)^{-1} R_{-} \\
R_{+} & 0
\end{array}\right)\left(\begin{array}{c}
u \\
u_{-}
\end{array}\right)=\left(\begin{array}{c}
(P-z)^{-1} v \\
v_{+}
\end{array}\right)
$$

and the matrix appearing here is injective. On the other hand it is a finite rank perturbation of the identity in $L^{2} \times \mathbf{C}^{N}$ and the injectivity implies the bijectivity. 
It follows that $\mathcal{P}(z)$ is bijective for $z \in \Omega(h) \cap \rho(P)$. Combining this with (3.7) and recalling that $\Omega$ is connected we see by a standard argument that $\mathcal{P}(z)$ is bijective for all $z \in \Omega$ and that $\left\|\mathcal{P}(z)^{-1}\right\| \leq C(h)$.

\section{Geometric preparations}

In this section we construct a special bounded weight that we shall implement in Section 5 with the help of Fourier integral operators with complex phase.

Let $P$ be the Operator introduced in the beginning of Section 2, satisfying (2.1)-(2.7). The symbol

$$
\widetilde{p}(x, \xi)=p_{0}(x)+\frac{p_{2}(x, \xi)}{\langle\xi\rangle^{2}}
$$

is non-negative and satisfies

$$
\left(\partial_{x},\langle\xi\rangle \partial_{\xi}\right)^{\alpha} \widetilde{p}=\mathcal{O}(1), \quad|\alpha| \geq 2,
$$

locally uniformly with respect to $x$. It follows that

$$
\left(\partial_{x},\langle\xi\rangle \partial_{\xi}\right)^{\alpha} \widetilde{p}=\mathcal{O}\left(\widetilde{p}^{\frac{1}{2}}\right), \quad|\alpha|=1 .
$$

We now introduce a critical set associated to $p$.

Hypothesis 4.1. Assume

The set $\left\{x \in M ; p_{0}(x)=0, \nu\left(x, \partial_{x}\right)=0\right\}$ is finite $=\left\{x_{1}, \ldots, x_{N}\right\}$.

Let $\rho_{j}=\left(x_{j}, 0\right)$ and put

$$
\mathcal{C}=\left\{\rho_{1}, \ldots, \rho_{N}\right\} .
$$

Notice that $p_{1}, p_{0}, p_{2}, \widetilde{p}$ vanish to second order at each $\rho_{j}$. Our weight will be of the form

$$
\psi_{\epsilon}=-\int k\left(\frac{t}{T_{0}}\right) \widetilde{p}_{\epsilon} \circ \exp \left(t H_{p_{1}}\right) d t, \quad 0<\epsilon \ll 1,
$$

where $\widetilde{p}_{\epsilon}$ will be specified below and $k$ is the same function as in Section 2. Notice that

$$
H_{p_{1}} \psi_{\epsilon}=\left\langle\widetilde{p}_{\epsilon}\right\rangle_{T_{0}}-\widetilde{p}_{\epsilon},
$$

where we now write in general,

$$
\langle q\rangle_{T_{0}}=\frac{1}{T_{0}} \int_{-T_{0} / 2}^{T_{0} / 2} q \circ \exp \left(t H_{p_{1}}\right) d t .
$$

Let $g(t) \in C^{\infty}([0,+\infty[;[0,1])$ be a smooth decreasing function with

$$
g(t)=\left\{\begin{array}{l}
1, \quad 0 \leq t \leq 1, \\
t^{-1}, \quad t \geq 2 .
\end{array}\right.
$$

Notice that $g^{(k)}(t)=\mathcal{O}\left(\langle t\rangle^{-1-k}\right)$. In a domain $0 \leq\left|\rho-\rho_{j}\right|\langle 1 / C, C \gg 1$, we put

$$
\widetilde{p}_{\epsilon}(\rho)=g\left(\frac{\left|\rho-\rho_{j}\right|^{2}}{\epsilon}\right) \widetilde{p},
$$


(so that $\widetilde{p}_{\epsilon}(\rho)=\widetilde{p}(\rho)$ for $\left.\left|\rho-\rho_{j}\right| \leq \sqrt{\epsilon}\right)$. It is easy to show the symbol estimates

$$
\partial^{\alpha} \widetilde{p}_{\epsilon}=\mathcal{O}\left(\frac{\epsilon}{\left(\epsilon+\left|\rho-\rho_{j}\right|^{2}\right)^{|\alpha| / 2}}\right), \quad 0 \leq\left|\rho-\rho_{j}\right|<1 / C
$$

Away from any fixed neighborhood of $\mathcal{C}$, we simply get

$$
\partial^{\alpha} \widetilde{p}_{\epsilon}=\mathcal{O}(\epsilon) .
$$

Let $\chi_{j} \in C_{0}^{\infty}\left(\mathbf{R}^{2 n}\right)$ be equal to 1 near $\rho_{j}$ and have its support close to $\mathcal{C}$. To define $\widetilde{p}_{\epsilon}$ further out from $\mathcal{C}$, we put

$$
\widetilde{p}_{\epsilon}(\rho)=\sum_{1}^{N} \chi_{j}(\rho) g\left(\frac{\left|\rho-\rho_{j}\right|^{2}}{\epsilon}\right) \widetilde{p}+\epsilon\left(1-\sum \chi_{j}(\rho)\right) \widetilde{p},
$$

so that (4.10) remains valid in a neighborhood of $\mathcal{C}$, while

$$
\partial_{x}^{\alpha} \partial_{\xi}^{\beta} \widetilde{p}_{\epsilon}=\mathcal{O}\left(\epsilon\langle\xi\rangle^{-|\beta|}\right)=\mathcal{O}\left(\epsilon\langle\xi\rangle^{-|\beta|}\right)
$$

outside any such region where $|x|$ is bounded. We also have

$$
\partial_{x}^{\alpha} \partial_{\xi}^{\beta}\left(\xi \cdot \partial_{\xi} \tilde{p}_{\epsilon}\right)=\mathcal{O}\left(\epsilon\langle\xi\rangle^{-2-|\beta|}\right)
$$

in the same region. In fact, (4.13) follows if we write for $|\xi| \gg 1$ :

$$
\xi \cdot \partial_{\xi} \widetilde{p}_{\epsilon}=\epsilon \xi \cdot \partial_{\xi} \frac{p_{2}}{\langle\xi\rangle^{2}}=\epsilon \xi \cdot \partial_{\xi}\left[\frac{p_{2}}{|\xi|^{2}}\left(1-\frac{1}{\langle\xi\rangle^{2}}\right)\right]=-\epsilon \frac{p_{2}}{|\xi|^{2}} \xi \cdot \partial_{\xi}\left(\frac{1}{\langle\xi\rangle^{2}}\right) .
$$

From (4.11) we see that $\widetilde{p}_{\epsilon}(\rho)=\epsilon \widetilde{p}$ when $\operatorname{dist}(\rho, \mathcal{C}) \geq 1 / C$ and in particular for $\rho=(x, \xi)$ with $\left|x-x_{j}\right| \geq 1 / C, \forall j$. Further away from $\left\{x_{1}, \ldots, x_{N}\right\}$ we want to make $\widetilde{p}_{\epsilon}(x, \xi)$ independent of $\xi$, so we replace $\widetilde{p}=p_{0}(x)+p_{2}(x, \xi) /\langle\xi\rangle^{2}$ in (4.11) by

$$
\widetilde{p}_{\text {new }}(x, \xi)=p_{0}(x)+\frac{\chi(x)}{\langle\xi\rangle^{2}} p_{2}(x, \xi),
$$

where $\chi \in C_{0}^{\infty}(M ;[0,1])$ is equal to 1 near $\left\{x_{1}, \ldots, x_{N}\right\}$.

Even further out (in the case when $M=\mathbf{R}^{n}$ ) we want to avoid problems caused by $p_{0}$ being large, so when $|x| \gg 1$ we want to replace $\widetilde{p}_{\epsilon}=\epsilon p_{0}$ there by $f_{\epsilon}\left(p_{0}\right)$, where $f_{\epsilon}(t)=\epsilon f(t / \epsilon)$ is the function introduced in Section 2. Thus with a new cutoff function $\chi_{\text {new }} \in C_{0}^{\infty}(M ;[0,1])$ being equal to 1 in a large neighborhood of $\operatorname{supp} \chi$, we get the final choice of $\widetilde{p}_{\epsilon}$ :

$$
\begin{aligned}
\widetilde{p}_{\epsilon}(\rho)= & \chi_{\text {new }}(x)\left(\sum_{1}^{N} \chi_{j}(\rho) g\left(\frac{\left|\rho-\rho_{j}\right|^{2}}{\epsilon}\right) \widetilde{p}+\epsilon\left(1-\sum \chi_{j}(\rho)\right) \widetilde{p}_{\text {new }}\right) \\
& +\left(1-\chi_{\text {new }}(x)\right) f_{\epsilon}\left(p_{0}\right) .
\end{aligned}
$$

Notice that by construction

$$
\tilde{p}_{\epsilon} \leq \tilde{p} \leq p_{0}+p_{2}
$$

We also get 
Proposition 4.2. We have (4.10) near $\mathcal{C}$ and (4.12), (4.13) in any closed subset of $T^{*} M$ disjoint from $\mathcal{C}$ and $\operatorname{supp}\left(1-\chi_{\text {new }}\right) \times \mathbf{R}^{n}$, where $\chi_{\text {new }}$ is the wider cutoff in (4.15). Over $\operatorname{supp}\left(1-\chi_{\text {new }}\right)$ (where $\widetilde{p}_{\epsilon}$ only depends on $x$ ) we have $\partial_{x}^{\alpha} \widetilde{p}_{\epsilon}=$ $\mathcal{O}\left(\epsilon^{1-|\alpha| / 2}\right)$.

From the definition (4.6), we see that $\psi_{\epsilon}$ satisfies the same estimates as $\widetilde{p}_{\epsilon}$ and will only depend on $x$ for $x$ outside a neighborhood $\pi_{x}(\mathcal{C})$ (that we can choose as small as we like) and that the region where we only have $\partial_{x}^{\alpha} \psi_{\epsilon}(x)=\mathcal{O}\left(\epsilon^{1-|\alpha| / 2}\right)$ can be any neighborhood of infinity. This follows from the fact that our various symbol estimates (as well as the $\xi$-independence) are conserved by the flow of $H_{p_{1}}$, as for (4.13), we here also use that $H_{p_{1}}$ commutes with dilations in $\xi$.

It also follows from the construction that

$$
\begin{array}{llll}
\widetilde{p}_{\epsilon}(\rho)=\widetilde{p}(\rho), & \text { when } & & \left|\rho-\rho_{j}\right| \leq \sqrt{\epsilon}, \\
\widetilde{p}_{\epsilon}(\rho) \backsim \frac{\epsilon}{\left|\rho-\rho_{j}\right|^{2}} \widetilde{p}, & \text { when } & \sqrt{\epsilon} \leq\left|\rho-\rho_{j}\right| \leq 1 / C, \\
\widetilde{p}_{\epsilon}(\rho) \backsim \epsilon \widetilde{p}_{\text {new }}, & \text { when } & \operatorname{dist}(\rho, \mathcal{C}) \geq 1 / C, \quad\left|\rho_{x}\right| \leq C, \\
\widetilde{p}_{\epsilon}(\rho)=\epsilon f_{\epsilon}\left(p_{0}\right), & \text { when } & \left|\rho_{x}\right| \geq \widetilde{C} .
\end{array}
$$

We introduce the following dynamical conditions where $T_{0}>0$ is fixed:

\section{Hypothesis 4.3.}

Near each $\rho_{j}$ we have $\langle\widetilde{p}\rangle_{T_{0}} \geq \frac{1}{C}\left|\rho-\rho_{j}\right|^{2}$,

In any set $|x| \leq C, \operatorname{dist}(\rho, \mathcal{C}) \geq \frac{1}{C}$,

$$
\text { we have }\langle\widetilde{p}\rangle_{T_{0}}(\rho) \geq \frac{1}{\widetilde{C}(C)}, \widetilde{C}(C)>0 \text {. }
$$

When $M=\mathbf{R}^{n}$ we also need a modified dynamical assumption

$\forall$ neighborhood $U$ of $\pi_{x} \mathcal{C}$, and $\forall x \in \mathbf{R}^{n} \backslash U, \exists C>0$,

$$
\text { meas }\left(\left\{t \in\left[-\frac{T_{0}}{2}, \frac{T_{0}}{2}\right] ; p_{0}(\exp t \nu(x)) \geq \frac{1}{C}\right\}\right) \geq \frac{1}{C} \text {. }
$$

When $M$ is compact, we just assume (4.21), (4.22), where it is understood that the estimate in (4.22) should hold for all $\rho \in T^{*} M$ of distance $\geq 1 / C$ from $\mathcal{C}$.

Notice that in the region $\operatorname{dist}\left(x, \pi_{x}(\mathcal{C})\right) \geq 1 / C,|x| \leq C,(4.22)$ is equivalent (up to a change of $\widetilde{C}(C)$ ) to

$$
\left\langle p_{0}\right\rangle_{T_{0}, \nu}(x) \geq \frac{1}{\widetilde{C}(C)}, \quad \widetilde{C}(C)>0,
$$

where

$$
\langle q\rangle_{T_{0}, \nu}=\frac{1}{T_{0}} \int_{-T_{0} / 2}^{T_{0} / 2} q \circ \exp (t \nu) d t
$$


This follows from the fact that $\pi_{x} \circ \exp t H_{p_{1}}=\exp (t \nu) \circ \pi_{x}$, where $\pi_{x}((x, \xi)):=x$ and the fact that $\{\xi=0\}$ is invariant under the $H_{p_{1}}$ flow.

Actually, if we only assume (4.21), then we see that $\left\langle p_{2}\right\rangle_{T_{0}}\left(x_{j}, \xi\right) \backsim \xi^{2}$, and if we also assume (4.24), we recover (4.22), first over a neighborhood of each $x_{j}$ and then again by (4.24) over any bounded set in $M$.

Assuming the dynamical conditions (4.21), (4.22), ((4.24), (4.23)), we see that

$$
\begin{aligned}
\left\langle\widetilde{p}_{\epsilon}\right\rangle_{T_{0}} & \sim \operatorname{dist}(\rho, \mathcal{C})^{2}, & \operatorname{dist}(\rho, \mathcal{C}) \leq \sqrt{\epsilon}, \\
\left\langle\widetilde{p}_{\epsilon}\right\rangle_{T_{0}} \backsim \epsilon, & & \operatorname{dist}(\rho, \mathcal{C})>\sqrt{\epsilon} .
\end{aligned}
$$

From the estimates on $\psi_{\epsilon}$ in the various regions that we mentioned after Proposition 4.2 we shall often only retain that

$$
\partial_{x}^{\alpha} \partial_{\xi}^{\beta} \psi_{\epsilon}(x, \xi)=\mathcal{O}\left(\epsilon^{1-|\alpha+\beta| / 2}\langle\xi\rangle^{-|\beta|}\right),
$$

and we write this for short as

$$
\psi_{\epsilon}=\widetilde{\mathcal{O}}(\epsilon) .
$$

Similarly, we have in view of (4.13), and (4.10) also valid for $\psi_{\epsilon}$, that

$$
\xi \cdot \partial_{\xi} \psi_{\epsilon}=\widetilde{\mathcal{O}}\left(\epsilon\langle\xi\rangle^{-2}\right) .
$$

From [20] let us recall that if $f$ is a $C^{1}$ function locally defined on complexified phase space, then at every point where $\bar{\partial} f=0$, we have

$$
\widehat{H}_{f}=H_{\operatorname{Re} f}^{\operatorname{Re} \sigma}=H_{\mathrm{I} m f}^{\operatorname{Im} \sigma}, \quad \widehat{H}_{i f}=J \widehat{H}_{f}=H_{-\mathrm{I} m f}^{\operatorname{Re} \sigma}=H_{\operatorname{Re} f}^{\operatorname{Im} \sigma},
$$

where

$$
H_{f}=\sum\left(\frac{\partial f}{\partial \xi_{j}} \frac{\partial}{\partial x_{j}}-\frac{\partial f}{\partial x_{j}} \frac{\partial}{\partial \xi_{j}}\right)
$$

is the complex $(1,0)$ Hamilton field of $f$ with respect to the complex symplectic form $\sigma=\sum d \xi_{j} \wedge d x_{j}$ and $\widehat{H}_{f}=H_{f}+\overline{H_{f}}$ is the real vector field which acts as $H_{f}$ on holomorphic functions. $H_{\mathrm{I} m f}^{\operatorname{Im} \sigma}$ denotes the real Hamilton field of $\operatorname{Im} f$ with respect to the real symplectic form I $m \sigma$, and similarly for the other Hamilton fields appearing in (4.29). As usual, $J$ denotes the action on tangent vectors induced by multiplication by $i$. (When $M$ is compact we may assume without loss of generality that $M$ is real-analytic.)

Assume first that $M=\mathbf{R}^{n}$ and put $\Lambda_{0}=T^{*} \mathbf{R}^{n}=\mathbf{R}^{2 n}$,

$$
\Lambda_{s}=\left\{\rho+i s H_{\psi_{\epsilon}}(\rho) ; \rho \in \Lambda_{0}\right\}, \quad 0 \leq s \ll 1 .
$$

If we extend $\psi_{\epsilon}$ to be a function on the complexified phase space $\mathbf{C}^{2 n}$, by setting

$$
\psi_{\epsilon}(\rho)=\psi_{\epsilon}(\operatorname{Re} \rho),
$$

then we have the equivalent description of $\Lambda_{s}$ as

$$
\Lambda_{s}=\exp \left(s H_{\psi_{\epsilon}}^{\mathrm{Im} \sigma}\right)\left(\Lambda_{0}\right) .
$$

It follows that $\Lambda_{s}$ is an I-Lagrangian manifold, i.e., a manifold which is Lagrangian for $\operatorname{I} m \sigma$. 
Now we would like to parametrize $\Lambda_{s}$ by means of a canonical transformation $\kappa(s): \Lambda_{0} \rightarrow \Lambda_{s}$ for the symplectic form Re $\sigma$ (implying that $\Lambda_{s}$ like $\Lambda_{0}$ is an IRmanifold, i.e., a manifold which is Lagrangian for $\operatorname{I} m \sigma$ and symplectic for the restriction of $\operatorname{Re} \sigma)$. For $0 \leq s \ll 1$, let $\widehat{\psi}_{\epsilon, s}=\widehat{\psi}(s, \rho)$ be the unique function which is affine in $\mathrm{I} m \rho$ and satisfies

$$
\bar{\partial}_{\rho} \widehat{\psi}_{\epsilon, s}=0 \quad \text { on } \quad \Lambda_{s}, \quad \widehat{\psi}_{\epsilon, s}=\psi_{\epsilon} \quad \text { on } \quad \Lambda_{s} .
$$

Recalling that $\psi_{\epsilon}(\rho)=\psi_{\epsilon}(\operatorname{Re} \rho)$, we get with the notation $\left(\psi_{\epsilon}\right)_{\xi}^{\prime}=\frac{\partial}{\partial \operatorname{Re} \xi} \psi_{\epsilon},\left(\psi_{\epsilon}\right)_{x}^{\prime}=$ $\frac{\partial}{\partial \operatorname{Re} x} \psi_{\epsilon}$ to indicate derivatives in the real directions,

$$
\begin{aligned}
\widehat{\psi}_{\epsilon, s}(x, \xi)= & \psi_{\epsilon}(x, \xi)+i a_{x}(\operatorname{Re}(x, \xi)) \cdot\left(\operatorname{Im} x-s\left(\psi_{\epsilon}\right)_{\xi}^{\prime}\right) \\
& +i a_{\xi}(\operatorname{Re}(x, \xi)) \cdot\left(\operatorname{Im} \xi+s\left(\psi_{\epsilon}\right)_{x}^{\prime}\right)
\end{aligned}
$$

where

$$
\left(1-i s^{t} F_{\psi_{\epsilon}}(\operatorname{Re}(x, \xi))\right)\left(\begin{array}{c}
a_{x} \\
a_{\xi}
\end{array}\right)=\left(\begin{array}{c}
\left(\psi_{\epsilon}\right)_{x}^{\prime} \\
\left(\psi_{\epsilon}\right)_{\xi}^{\prime}
\end{array}\right)
$$

and

$$
F_{\psi_{\epsilon}}=\left(\begin{array}{cc}
\left(\psi_{\epsilon}\right)_{\xi x}^{\prime \prime} & \left(\psi_{\epsilon}\right)_{\xi \xi}^{\prime \prime} \\
-\left(\psi_{\epsilon}\right)_{x x}^{\prime \prime} & -\left(\psi_{\epsilon}\right)_{x \xi}^{\prime \prime}
\end{array}\right)
$$

is the fundamental matrix of $\psi_{\epsilon}$. It also follows from the construction that

$$
a_{x}=\widetilde{\mathcal{O}}\left(\epsilon^{\frac{1}{2}}\right), \quad a_{\xi}=\widetilde{\mathcal{O}}\left(\epsilon^{\frac{1}{2}}\langle\xi\rangle^{-1}\right) .
$$

Since $\widehat{\psi}_{\epsilon, s}=\psi_{\epsilon}$ on $\Lambda_{s}$, we know that $H_{\operatorname{Re} \widehat{\psi}_{\epsilon, s}}^{\mathrm{Im} \sigma}-H_{\psi_{\epsilon}}^{\mathrm{Im} \sigma}$ is tangent to $\Lambda_{s}$ for every $s$. We can therefore define $\kappa(s): \Lambda_{0} \rightarrow \Lambda_{s}$, by

$$
\frac{d}{d s} \kappa(s)(\rho)=H_{\operatorname{Re} \widehat{\psi}_{\epsilon, s}}^{\operatorname{Im} \sigma}(\kappa(s)(\rho)), \quad \rho \in \Lambda_{0} .
$$

The second relation in (4.29) implies that $H_{\operatorname{Re} \widehat{\psi}_{\epsilon, s}}^{\operatorname{Im} \sigma}=H_{-\operatorname{Im} \widehat{\psi}_{\epsilon, s}}^{\operatorname{Re}}$ on $\Lambda_{s}$ (given that $\bar{\partial} \widehat{\psi}_{\epsilon, s}=0$ there) and hence $\kappa(s)$ is symplectic for $\operatorname{Re} \sigma: \kappa(s)^{*}(\operatorname{Re} \sigma)=\operatorname{Re} \sigma$. Notice that (4.38) again shows that $\Lambda_{s}$ is an I-Lagrangian manifold.

A priori, $\kappa(s)(\rho)$ is well-defined only for $s \geq 0$ small enough depending on $\rho$, but we shall next derive symbol estimates for $\widehat{\psi}_{\epsilon, s}$ and $\kappa(s)$ that will imply that $\kappa(s)(\rho)$ is indeed well-defined for $s$ small enough independently of $\rho$. Assume that we work near a point $\left(x_{0}, r \xi_{0}\right), x_{0} \in \mathbf{R}^{n}, \xi_{0} \in S^{n-1}, r \geq 0$ and replace $(x, \xi)$ by $(\widetilde{x}, \widetilde{\xi})$, where

$$
x=x_{0}+\sqrt{\epsilon} \widetilde{x}, \quad \xi=r \xi_{0}+\langle r\rangle \sqrt{\epsilon} \widetilde{\xi} .
$$

Define $\widetilde{\psi}=\widetilde{\psi}_{\epsilon, x_{0}, r, \xi_{0}}$ by $\psi_{\epsilon}(x, \xi)=\epsilon \widetilde{\psi}(\widetilde{x}, \widetilde{\xi})$, so that

$$
\begin{aligned}
& \widetilde{\psi}(\widetilde{x}, \widetilde{\xi})=\frac{1}{\epsilon} \psi_{\epsilon}\left(x_{0}+\sqrt{\epsilon} \widetilde{x}, r \xi_{0}+\langle r\rangle \sqrt{\epsilon} \widetilde{\xi}\right) \\
& \partial_{\widetilde{x}}^{\alpha} \partial_{\widetilde{\xi}}^{\beta} \widetilde{\psi}=\mathcal{O}(1), \quad \forall \alpha, \beta, \text { for }|(\widetilde{x}, \widetilde{\xi})| \leq \text { Const. }
\end{aligned}
$$


In the new coordinates $\Lambda_{s}$ takes the form

$$
\operatorname{Im}(\widetilde{x}, \widetilde{\xi})=\frac{s}{\langle r\rangle} H_{\widetilde{\psi}}(\operatorname{Re}(\widetilde{x}, \widetilde{\xi})) .
$$

The scaling and the construction of $\widehat{\psi}_{\epsilon, s}$ commute so if we put

$$
\widetilde{\widehat{\psi}}_{\epsilon, s}=\frac{1}{\epsilon} \widehat{\psi}_{\epsilon, s}\left(x_{0}+\sqrt{\epsilon} \widetilde{x}, r \xi_{0}+\langle r\rangle \sqrt{\epsilon} \widetilde{\xi}\right),
$$

then (4.41) still holds. In particular $\widehat{\psi}_{\epsilon, s}$ satisfies the same estimates on $\Lambda_{s}$ as $\psi_{\epsilon}$ in (4.27), now with $\partial_{x}, \partial_{\xi}$ replaced by $\left(\partial_{\operatorname{Re} x}, \partial_{\operatorname{Im} x}\right),\left(\partial_{\operatorname{Re} \xi}, \partial_{\operatorname{Im} \xi}\right)$.

The transformation $\kappa(s)$ can be scaled similarly and the scaling commutes with (4.38) up to a factor $\langle r\rangle$ : If we put

$$
\kappa(s)(\rho)=(x(s), \xi(s))=\left(x_{0}+\sqrt{\epsilon} \widetilde{x}(s), r \xi_{0}+\langle r\rangle \sqrt{\epsilon} \widetilde{\xi}(s)\right),
$$

then from (4.38), (4.29) we get

$$
\begin{aligned}
& \partial_{s} \widetilde{x}(s)=\frac{i}{\langle r\rangle} \partial_{\widetilde{\xi}} \widetilde{\widehat{\psi}}_{\epsilon, s}(\widetilde{x}(s), \widetilde{\xi}(s)), \\
& \partial_{s} \widetilde{\xi}(s)=-\frac{i}{\langle r\rangle} \partial_{\widetilde{x}} \widetilde{\widehat{\psi}}_{\epsilon, s}(\widetilde{x}(s), \widetilde{\xi}(s)) .
\end{aligned}
$$

We conclude that with $(x(0), \xi(0))=(y, \eta)=\left(x_{0}+\sqrt{\epsilon} \widetilde{y}, r \xi_{0}+\langle r\rangle \sqrt{\epsilon} \widetilde{\eta}\right)$,

$$
\partial_{s}^{k} \partial_{\widetilde{y}}^{\alpha} \partial_{\widetilde{\eta}}^{\beta}(\widetilde{x}, \widetilde{\xi})=\mathcal{O}\left(\langle r\rangle^{-k}\right)
$$

and hence for $(x, \xi)=\kappa(s)(y, \eta)$ :

$$
\begin{aligned}
& \partial_{s}^{k} \partial_{y}^{\alpha} \partial_{\eta}^{\beta} x=\mathcal{O}\left(\epsilon^{\frac{1}{2}-\frac{|\alpha+\beta|}{2}}\langle\eta\rangle^{-k-|\beta|}\right), \\
& \partial_{s}^{k} \partial_{y}^{\alpha} \partial_{\eta}^{\beta} \xi=\mathcal{O}\left(\epsilon^{\frac{1}{2}-\frac{|\alpha+\beta|}{2}}\langle\eta\rangle^{1-k-|\beta|}\right),
\end{aligned}
$$

when $k+|\alpha+\beta| \geq 1$.

Notice that the right hand sides in (4.43) reduce to $i\langle r\rangle^{-1} \partial_{\widetilde{\xi}} \widetilde{\psi}$ and $-i\langle r\rangle^{-1} \partial_{\widetilde{x}} \widetilde{\psi}$ when $s=0$, where the derivatives are taken in the real directions. The flow in (4.45) is therefore tangent to the one in (4.32) at $s=0$ and we get

$$
\begin{aligned}
\kappa(s)(y, \eta) & =(y, \eta)+i s H_{\psi_{\epsilon}}(y, \eta)+(z, \zeta), \\
\partial_{y}^{\alpha} \partial_{\eta}^{\beta} z & =\mathcal{O}\left(\epsilon^{\frac{1}{2}-\frac{|\alpha+\beta|}{2}} s^{2}\langle\eta\rangle^{-2-|\beta|}\right), \\
\partial_{y}^{\alpha} \partial_{\eta}^{\beta} \zeta & =\mathcal{O}\left(\epsilon^{\frac{1}{2}-\frac{|\alpha+\beta|}{2}} s^{2}\langle\eta\rangle^{-1-|\beta|}\right) .
\end{aligned}
$$

From (4.45) and the subsequent remark, we have for $\kappa(\delta)(x, \xi)=\kappa(\delta, x, \xi)$.

$$
\kappa(\delta, x, \xi)=(x, \xi)+i \delta H_{\psi_{\epsilon}}(x, \xi)+\epsilon^{\frac{1}{2}} \delta^{2}\left(\widetilde{\mathcal{O}}\left(\frac{1}{\langle\xi\rangle^{2}}\right), \widetilde{\mathcal{O}}\left(\frac{1}{\langle\xi\rangle}\right)\right) .
$$

Recalling how $\kappa(\delta)$ was constructed we conclude that

$$
\kappa(\delta, x, \xi)=(\widetilde{x}, \widetilde{\xi})+i \delta H_{\psi_{\epsilon}}(\widetilde{x}, \widetilde{\xi}),
$$

where $(\widetilde{x}, \widetilde{\xi})$ is real and $(\widetilde{x}, \widetilde{\xi})-(x, \xi)=\epsilon^{\frac{1}{2}} \delta^{2}\left(\widetilde{\mathcal{O}}\left(\frac{1}{\langle\xi\rangle^{2}}\right), \widetilde{\mathcal{O}}\left(\frac{1}{\langle\xi\rangle}\right)\right)$. Put $\alpha_{\delta}(x, \xi)=(\widetilde{x}, \widetilde{\xi})$. 
The essential part of the discussion above took part near the points of $\mathcal{C}$. In that region the discussion is the same in the case when $M$ is compact.

\section{Quantization of weights}

We will follow $[12,23]$ with one modification; instead of analyticity we will use that our weights are "moderate" allowing us to use almost holomorphic extensions. Another minor difference is that we shall not use FBI-transforms explicitly, but rather rely on certain Fourier integral operators with complex phase. We will assume that $M=\mathbf{R}^{n}$ for simplicity, but as in the preceding section the essential part of the work will take place near $\mathcal{C}$ and here there is no difference between the case $M=\mathbf{R}^{n}$ and the case when $M$ is compact.

As a first step towards introducing some Fourier integral operators we shall study the function $h(y, \eta)$ on $T^{*} \mathbf{R}^{n}$, given by

$$
\kappa(\delta)^{*}(\xi \cdot d x)-\eta \cdot d y=d h .
$$

Recall here that

$$
\frac{d}{d s} \kappa(s) \rho=H_{i \widehat{\psi}_{\epsilon, s}}(\kappa(s) \rho), \quad \rho \in \Lambda_{0}=T^{*} \mathbf{R}^{n},
$$

where $\widehat{\psi}_{\epsilon, s}$ is given by (4.34), satisfying (4.33). Of course, (5.2) remains unchanged if we replace $\widehat{\psi}_{\epsilon, s}$ by an almost holomorphic extension from $\Lambda_{s}=\Lambda_{s \psi_{\epsilon}}$. Now using Cartan's formula, we get

$$
\begin{aligned}
\frac{d}{d s} \kappa(s)^{*}(\xi \cdot d x) & =\kappa(s)^{*} \mathcal{L}_{i H_{\widehat{\psi}_{\epsilon, s}}}(\xi \cdot d x) \\
& \left.\left.=i \kappa(s)^{*}\left(H_{\widehat{\psi}_{\epsilon, s}}\right\rfloor d(\xi \cdot d x)+d\left(H_{\widehat{\psi}_{\epsilon, s}}\right\rfloor \xi \cdot d x\right)\right) \\
& \left.=i \kappa(s)^{*}\left(H_{\widehat{\psi}_{\epsilon, s}}\right\rfloor \sigma+d\left(\xi \cdot \frac{\partial \widehat{\psi}_{\epsilon, s}}{\partial \xi}\right)\right) \\
& =i \kappa(s)^{*}\left(d\left(\xi \cdot \frac{\partial \widehat{\psi}_{\epsilon, s}}{\partial \xi}\right)-d \widehat{\psi}_{\epsilon, s}\right) \\
& =i d \kappa(s)^{*}\left(\xi \cdot \frac{\partial}{\partial \xi} \widehat{\psi}_{\epsilon, s}-\widehat{\psi}_{\epsilon, s}\right) .
\end{aligned}
$$

Thus, we can take

$$
h=i \int_{0}^{\delta}\left(\xi \cdot \partial \partial \partial \xi \widehat{\psi}_{\epsilon, s}-\widehat{\psi}_{\epsilon, s}\right) \circ \kappa(s) d s .
$$

On $\Lambda_{s}$ we have $\frac{\partial \widehat{\psi}_{\epsilon, s}}{\partial \xi}=\frac{\partial \widehat{\psi}_{\epsilon, s}}{\partial \operatorname{Re} \xi}$ and we recall that $\psi_{\epsilon}(x, \xi)=\psi_{\epsilon}(\operatorname{Re}(x, \xi))$. Using (4.34), we get on $\Lambda_{s}$ :

$$
\begin{aligned}
\xi \cdot \frac{\partial}{\partial \xi} \widehat{\psi}_{\epsilon, s}= & \xi \cdot \frac{\partial}{\partial \operatorname{Re} \xi} \psi_{\epsilon}(x, \xi)-i s\left\langle a_{x}(\operatorname{Re}(x, \xi)) \mid\left(\psi_{\epsilon}\right)_{\xi \xi}^{\prime \prime} \xi\right\rangle \\
& +i s\left\langle a_{\xi}(\operatorname{Re}(x, \xi)) \mid\left(\psi_{\epsilon}\right)_{x \xi}^{\prime \prime} \xi\right\rangle
\end{aligned}
$$


where again we use the notation $(\cdot)_{\xi}^{\prime},(\cdot)_{x \xi}^{\prime \prime}$ etc to indicate derivatives in the real directions.

We now estimate the terms in the right hand side of (5.4). On $\Lambda_{s}$ we have $\operatorname{Im} \xi=s\left(\psi_{\epsilon}\right)_{\xi}^{\prime}$, so

$$
\xi \cdot \frac{\partial}{\partial \operatorname{Re} \xi} \psi_{\epsilon}=\operatorname{Re} \xi \cdot\left(\psi_{\epsilon}\right)_{\xi}^{\prime}-i s\left(\psi_{\epsilon}\right)_{x}^{\prime} \cdot\left(\psi_{\epsilon}\right)_{\xi}^{\prime} \cdot
$$

Hence by (4.27), (4.28),

$$
\xi \cdot \frac{\partial}{\partial \operatorname{Re} \xi} \psi_{\epsilon}=\widetilde{\mathcal{O}}\left(\epsilon\langle\xi\rangle^{-1}\right) .
$$

Next, look at

$$
\left\langle a_{x} \mid\left(\psi_{\epsilon}\right)_{\xi \xi}^{\prime \prime} \xi\right\rangle=\left\langle a_{x} \mid\left(\psi_{\epsilon}\right)_{\xi \xi}^{\prime \prime} \operatorname{Re} \xi\right\rangle-i s\left\langle a_{x} \mid\left(\psi_{\epsilon}\right)_{\xi \xi}^{\prime \prime}\left(\psi_{\epsilon}\right)_{x}^{\prime}\right\rangle .
$$

The last term is $\widetilde{\mathcal{O}}\left(\epsilon\langle\xi\rangle^{-2}\right)$ by (4.27), (4.37). The first term is equal to

$$
\left\langle a_{x} \mid\left(\psi_{\epsilon}\right)_{\xi \xi}^{\prime \prime} \operatorname{Re} \xi\right\rangle=a_{x} \cdot \frac{\partial}{\partial \operatorname{Re} \xi}\left(\left(\psi_{\epsilon}\right)_{\xi}^{\prime} \cdot \operatorname{Re} \xi\right)-\left(\psi_{\epsilon}\right)_{\xi}^{\prime} \cdot a_{x} .
$$

From (4.28), (4.27), (4.37) this is $\widetilde{\mathcal{O}}\left(\epsilon\langle\xi\rangle^{-1}\right)$, so

$$
\left\langle a_{x} \mid\left(\psi_{\epsilon}\right)_{\xi \xi}^{\prime \prime} \xi\right\rangle=\widetilde{\mathcal{O}}\left(\epsilon\langle\operatorname{Re} \xi\rangle^{-1}\right) .
$$

Similarly,

so

$$
\begin{aligned}
\left\langle a_{\xi} \mid\left(\psi_{\epsilon}\right)_{x \xi}^{\prime \prime} \xi\right\rangle & =\left\langle a_{\xi} \mid\left(\psi_{\epsilon}\right)_{x \xi}^{\prime \prime} \operatorname{Re} \xi\right\rangle-i s\left\langle a_{\xi} \mid\left(\psi_{\epsilon}\right)_{x \xi}^{\prime \prime}\left(\psi_{\epsilon}\right)_{x}^{\prime}\right\rangle \\
& =\left\langle a_{\xi} \mid\left(\psi_{\epsilon}\right)_{x \xi}^{\prime \prime} \operatorname{Re} \xi\right\rangle+\widetilde{\mathcal{O}}\left(\epsilon\langle\operatorname{Re} \xi\rangle^{-2}\right) \\
& =a_{\xi} \cdot \frac{\partial}{\partial \operatorname{Re} x}\left(\frac{\partial \psi_{\epsilon}}{\partial \operatorname{Re} \xi} \cdot \operatorname{Re} \xi\right)+\widetilde{\mathcal{O}}\left(\epsilon\langle\operatorname{Re} \xi\rangle^{-2}\right),
\end{aligned}
$$

$$
\left\langle a_{\xi} \mid\left(\psi_{\epsilon}\right)_{x \xi}^{\prime \prime} \xi\right\rangle=\widetilde{\mathcal{O}}\left(\epsilon\langle\operatorname{Re} \xi\rangle^{-2}\right) .
$$

Returning to (5.4), we get

$$
\xi \cdot \frac{\partial}{\partial \xi} \widehat{\psi}_{\epsilon, s}=\widetilde{\mathcal{O}}\left(\epsilon\langle\operatorname{Re} \xi\rangle^{-1}\right) \quad \text { on } \quad \Lambda_{s} .
$$

Also recall that $\widehat{\psi}_{\epsilon, s}=\psi_{\epsilon}$ on $\Lambda_{s}$, so

$$
\xi \cdot \frac{\partial}{\partial \xi} \widehat{\psi}_{\epsilon, s}-\widehat{\psi}_{\epsilon, s}=\widetilde{\mathcal{O}}(\epsilon) \quad \text { on } \quad \Lambda_{s} .
$$

Combining this with (4.45), we get

$$
\left(\xi \cdot \frac{\partial}{\partial \xi} \widehat{\psi}_{\epsilon, s}-\widehat{\psi}_{\epsilon, s}\right) \circ \kappa(s)=\widetilde{\mathcal{O}}(\epsilon),
$$

and finally from (5.3) we obtain

Lemma 5.1.

$$
h=\widetilde{\mathcal{O}}(\delta \epsilon) .
$$


Following [12] we shall now quantize $\kappa(\delta)$ by means of a Fourier integral operator

$$
A u(x ; h)=\iint_{(y, \alpha) \in \mathbf{R}^{n} \times T^{*} M} e^{\frac{i}{h} \phi(x, y, \alpha)} a(x, y, \alpha ; h) \chi\left(x, y, \alpha_{x}\right) u(y) d y d \alpha,
$$

with $a \in S^{\frac{3 n}{2}+m, \frac{n}{2}+k}$, and where $\chi$ is a standard cutoff to a neighborhood of the diagonal: $\left|x-\alpha_{x}\right|,\left|y-\alpha_{x}\right|<1 / C$, equal to 1 on a smaller neighborhood of the same type. Here we take with $\beta=\kappa(\delta, \alpha)=\kappa(\delta)(\alpha)$ :

$$
\phi(x, y, \alpha)=\left(x-\beta_{x}\right) \cdot \beta_{\xi}+\left(\alpha_{x}-y\right) \cdot \alpha_{\xi}+\psi\left(x-\beta_{x}, y-\alpha_{x}, \alpha\right)+h(\alpha),
$$

where on the real domain,

$$
\psi\left(x-\beta_{x}, y-\alpha_{x}, \alpha\right)=\mathcal{O}\left(\left\langle\alpha_{\xi}\right\rangle\left(\left(x-\beta_{x}\right)^{2}+\left(y-\alpha_{x}\right)^{2}\right)\right),
$$

in the symbol sense:

$$
\partial_{x}^{k} \partial_{y}^{\ell} \partial_{\alpha_{x}}^{m} \partial_{\alpha_{\xi}}^{p} \psi(x, y, \alpha)=\mathcal{O}\left(\left\langle\alpha_{\xi}\right\rangle^{1-|p|}(|x|+|y|)^{(2-|k+\ell|)_{+}}\right),
$$

and we take almost holomorphic extensions satisfying the same estimates in the complex domain. Further, $a \in S^{\frac{3 n}{2}+m, \frac{n}{2}+k}$ means that $a=\mathcal{O}\left(h^{-\frac{3 n}{2}-m}\left\langle\alpha_{\xi}\right\rangle^{\frac{n}{2}+k}\right)$ in the symbol sense, $\partial_{x, y}^{\ell} \partial_{\alpha_{x}}^{p} \partial_{\alpha_{\xi}}^{q} a(x, y, \alpha ; h)=\mathcal{O}\left(h^{-\frac{3 n}{2}-m}\left\langle\alpha_{\xi}\right\rangle^{\frac{n}{2}+k-|q|}\right)$.

We also assume that

$$
\operatorname{Im} \psi \backsim\left\langle\alpha_{\xi}\right\rangle\left(\left(x-\beta_{x}\right)^{2}+\left(y-\alpha_{x}\right)^{2}\right) .
$$

Viewing $h$ as a function on the graph of $\kappa(\delta)$, we have $d h=\beta_{\xi} \cdot d \beta_{x}-\alpha_{\xi} \cdot d \alpha_{x}$, so for

$$
x=\kappa(\delta, \alpha)_{x}, \quad y=\alpha_{x},
$$

we have $d_{\alpha} \phi=0$. Moreover, in a neighborhood of that set, we have with $\psi=$ $\psi(x, y, \alpha)$,

$$
\begin{aligned}
d_{\alpha} \phi= & \left(x-\kappa(\alpha)_{x}\right) \cdot d_{\alpha}\left(\kappa(\alpha)_{\xi}\right)+\left(\alpha_{x}-y\right) \cdot d \alpha_{\xi} \\
& -\frac{\partial \psi}{\partial x}\left(x-\kappa(\alpha)_{x}, y-\alpha_{x}, \alpha\right) \cdot d_{\alpha}\left(\kappa(\alpha)_{x}\right)-\frac{\partial \psi}{\partial y} \cdot d \alpha_{x}+\frac{\partial \psi}{\partial \alpha} \cdot d \alpha .
\end{aligned}
$$

So $\phi$ is a non-degenerate phase function in the sense of Hörmander (a part from the homogeneity condition in the fiber variables) with a critical set (5.16), the associated canonical transformation is $\kappa=\kappa(\delta)$.

Similarly, to $\kappa(\delta)^{-1}$ we can associate

$$
B v(x ; h)=\iint_{(y, \gamma) \in \mathbf{R}^{n} \times T^{*} M} e^{\frac{i}{h} \widehat{\phi}(x, y, \gamma)} b(x, y, \gamma ; h) \chi(x, y, \gamma) v(y) d y d \gamma,
$$

$b \in S^{\frac{3 n}{2}+\widehat{m}, \frac{n}{2}+\widehat{k}}, \widehat{m}, \widehat{k} \in \mathbf{R}$, where with $\beta=\kappa(\delta)(\gamma):$

$$
\widehat{\phi}(x, y, \gamma)=\left(x-\gamma_{x}\right) \cdot \gamma_{\xi}+\left(\beta_{x}-y\right) \cdot \beta_{\xi}+\widehat{\psi}\left(x-\gamma_{x}, y-\beta_{x}, \gamma\right)-h(\gamma)
$$

and $\widehat{\psi}$ satisfies (5.14). Again this is a non-degenerate phase and the critical set is given by

$$
x=\gamma_{x}, \quad y=\beta_{x}, \quad(\beta=\kappa(\delta)(\gamma)),
$$


and the associated canonical transformation is $\kappa(\delta)^{-1}$.

For $x, y \in \mathbf{R}^{n},\left|x-\alpha_{x}\right|,\left|y-\beta_{x}\right| \leq 1 / C$, we have with $\beta=\kappa(\alpha)=\kappa(\delta, \alpha)$ :

$$
\begin{aligned}
\operatorname{Im} \phi(x, y, \alpha)= & \left(x-\operatorname{Re} \beta_{x}\right) \cdot \operatorname{Im} \beta_{\xi}-\operatorname{Im} \beta_{x} \cdot \operatorname{Re} \beta_{\xi} \\
& +\operatorname{Im} \psi\left(x-\beta_{x}, y-\alpha_{x}, \beta, \alpha\right)+\operatorname{Im} h(\alpha) .
\end{aligned}
$$

Here

$$
\begin{aligned}
\operatorname{Im} \beta_{x} & =\delta \widetilde{\mathcal{O}}\left(\epsilon^{\frac{1}{2}\left\langle\operatorname{Re} \alpha_{\xi}\right\rangle^{-1}}\right), \quad \operatorname{Im} \beta_{\xi}=\delta \widetilde{\mathcal{O}}\left(\epsilon^{\frac{1}{2}}\right), \\
\operatorname{Im} \beta_{x} \cdot \operatorname{Re} \beta_{\xi} & =\delta \operatorname{Re} \beta_{\xi} \cdot \frac{\partial \psi_{\epsilon}}{\partial \operatorname{Re} \beta_{\xi}}=\widetilde{\mathcal{O}}\left(\frac{\delta \epsilon}{\left\langle\operatorname{Re} \beta_{\xi}\right\rangle^{2}}\right),
\end{aligned}
$$

and

$$
\begin{aligned}
\operatorname{Im} h(\alpha) & =\widetilde{\mathcal{O}}(\delta \epsilon), \\
\operatorname{Im} \psi\left(x-\beta_{x}, y-\alpha_{x}, \alpha\right) & \geq\left\langle\alpha_{\xi}\right\rangle\left(\frac{\left|x-\operatorname{Re} \beta_{x}\right|^{2} C}{+} \frac{1}{C}\left|y-\alpha_{x}\right|^{2}-C\left|\operatorname{Im} \beta_{x}\right|^{2}\right),
\end{aligned}
$$

so

$$
\begin{aligned}
\operatorname{Im} \phi(x, y, \alpha) \geq & \frac{\left\langle\alpha_{\xi}\right\rangle}{C}\left(\left|x-\operatorname{Re} \beta_{x}\right|^{2}+\left|y-\alpha_{x}\right|^{2}\right) \\
& -C \frac{\delta^{2} \epsilon\left\langle\alpha_{\xi}\right\rangle}{\left\langle\alpha_{\xi}\right\rangle^{2}}-C\left|x-\operatorname{Re} \beta_{x}\right| \delta \epsilon^{\frac{1}{2}}-C \delta \epsilon, \\
\operatorname{Im} \phi \geq & \frac{\left\langle\alpha_{\xi}\right\rangle}{2 C}\left(\left|x-\operatorname{Re} \beta_{x}\right|^{2}+\left|y-\alpha_{x}\right|^{2}\right)-\widetilde{C} \delta \epsilon .
\end{aligned}
$$

Recalling that $\epsilon=A h, A \gg 1$, we get

$$
\left|e^{\frac{i}{h} \phi(x, y, \alpha)}\right| \leq \exp \left(-\frac{\left\langle\alpha_{\xi}\right\rangle}{2 C h}\left(\left|x-\operatorname{Re} \beta_{x}\right|^{2}+\left|y-\alpha_{x}\right|^{2}\right)+\widetilde{C} \delta A\right) .
$$

A similar estimate holds for $\widehat{\phi}$. It follows that $A, B$ are well-defined Operators: $\mathcal{S} \rightarrow$ $\mathcal{S}$ with semi-norm estimates that are uniform in powers of $h$. Also for every $s \in \mathbf{R}$, there is an $s^{\prime} \in \mathbf{R}$ such that $A, B: H^{s} \rightarrow H^{s^{\prime}}$ with norms bounded by some power of $h$. Moreover, our Operators are independent of the choice of almost holomorphic extensions of the phase and amplitude modulo Operators whose integral kernels are $\mathcal{O}\left(h^{\infty}\right)$ with all their derivatives and supported in a domain of the form $|x-y| \leq$ $\mathcal{O}(1)$.

Proposition 5.2. If $m, k, \widehat{m}, \widehat{k}=0$, then $A, B=\mathcal{O}(\exp \mathcal{O}(1) \delta A): L^{2}\left(\mathbf{R}^{n}\right) \rightarrow$ $L^{2}\left(\mathbf{R}^{n}\right)$

The proof will be given later.

Proposition 5.3. We have $B A=\mathrm{Op}_{h}(c)$, where $c=\widetilde{\mathcal{O}}\left(h^{-m-\widehat{m}}\left\langle\alpha_{\xi}\right\rangle^{k+\widehat{k}}\right)$

Proof. We have

$$
B A u(x)=\iiint \int e^{\frac{i}{h}(\widehat{\phi}(x, z, \gamma)+\phi(z, y, \alpha))} b(x, z, \gamma ; h) a(z, y, \alpha ; h) u(y) d y d \alpha d z d \gamma,
$$


where the cutoffs $\chi, \widehat{\chi}$ have been incorporated in $a, b$. Here

$$
\begin{aligned}
\widehat{\phi}(x, z, \gamma)+\phi(z, y, \alpha)= & \left(x-\gamma_{x}\right) \cdot \gamma_{\xi}+\left(\kappa(\gamma)_{x}-z\right) \cdot \kappa(\gamma)_{\xi} \\
& +\left(z-\kappa(\alpha)_{x}\right) \cdot \kappa(\alpha)_{\xi} \\
& +\left(\alpha_{x}-y\right) \cdot \alpha_{\xi}+\widehat{\psi}\left(x-\gamma_{x}, z-\kappa(\gamma)_{x}, \gamma\right) \\
& +\psi\left(z-\kappa(\alpha)_{x}, y-\alpha_{x}, \alpha\right)+h(\alpha)-h(\gamma) .
\end{aligned}
$$

The contribution to the distribution kernel from a region with $\left|\gamma_{\xi}-\alpha_{\xi}\right| /\left\langle\alpha_{\xi}\right\rangle \geq$ $C^{-1}$ is $\mathcal{O}\left(h^{\infty}\right)$, as can be seen by integration by parts with respect to $z$. More precisely the distribution kernel of $B A$ is

$$
(B A)(x, y)=\iiint e^{\frac{i}{h}(\widehat{\phi}+\phi)} b a \chi\left(\frac{\left|\gamma_{\xi}-\alpha_{\xi}\right|}{\left\langle\alpha_{\xi}\right\rangle}\right) d \alpha d z d \gamma+R(x, y ; h),
$$

where $\chi \in C_{0}^{\infty}\left(\left[0, \infty[)\right.\right.$ is equal to 1 near 0 , and $\partial_{x}^{k} \partial_{y}^{\ell} R=\mathcal{O}\left(h^{\infty}\right)$. Here (5.22) and the analogous estimate for $e^{\frac{i}{h} \hat{\phi}}$ are essential of course.

Next using (5.21) and the similar estimate for $\operatorname{I} m \widehat{\phi}$, we get a localization in $\left|\gamma_{x}-\alpha_{x}\right|$, leading to

$$
\begin{aligned}
(B A)(x, y)= & \iiint e^{\frac{i}{h}(\widehat{\phi}+\phi)} b a \chi\left(\left(\gamma_{x}-\alpha_{x}\right)^{2}+\frac{\left(\gamma_{\xi}-\alpha_{\xi}\right)^{2}}{\left\langle\alpha_{\xi}\right\rangle^{2}}\right) d \alpha d z d \gamma \\
& +\widetilde{R}(x, y ; h),
\end{aligned}
$$

where $\partial_{x}^{k} \partial_{y}^{\ell} \widetilde{R}=\mathcal{O}\left(h^{\infty}\right)$. Here $\chi \in C_{0}^{\infty}\left(\left[0, \epsilon_{0}[), \chi=1\right.\right.$ near 0 , and $\epsilon_{0}$ can be any fixed number.

In the integral (5.24), we may assume that $\left|x-\gamma_{x}\right|,\left|y-\alpha_{x}\right|,\left|z-\kappa(\alpha)_{x}\right|<1 / C$, where $C$ is as large as we like, since the integral in the complementary region is exponentially small. We now want to eliminate integration variables by means of the method of stationary phase, and we start by carrying out the $z$-integration, so we first look for the critical point of

$$
z \mapsto \widehat{\phi}(x, z, \gamma)+\phi(z, y, \alpha),
$$

where $\phi, \widehat{\phi}$ also denote almost holomorphic extensions. Let $F_{\delta}(x, \gamma, y, \alpha)$ denote the corresponding critical value.

In order to understand this function, we first treat $\widetilde{\gamma}=\kappa(\gamma), \widetilde{\alpha}=\kappa(\alpha)$ as independent variables (writing $\kappa(\alpha)$ instead of $\kappa(\delta, \alpha)$ for short). Let

$$
\begin{aligned}
G(x, \gamma, \widetilde{\gamma}, y, \alpha, \widetilde{\alpha})= & \operatorname{vc}_{z}\left[\left(x-\gamma_{x}\right) \cdot \gamma_{\xi}+\left(\widetilde{\gamma}_{x}-z\right) \cdot \widetilde{\gamma}_{\xi}+\left(z-\widetilde{\alpha}_{x}\right) \cdot \widetilde{\alpha}_{\xi}+\left(\alpha_{x}-y\right) \cdot \alpha_{\xi}\right. \\
& \left.+\widehat{\psi}\left(x-\gamma_{x}, z-\widetilde{\gamma}_{x}, \gamma\right)+\psi\left(z-\widetilde{\alpha}_{x}, y-\alpha_{x}, \alpha\right)\right] .
\end{aligned}
$$

Here the critical point $z=z_{c}(x, \gamma, \widetilde{\gamma}, y, \alpha, \widetilde{\alpha})$ satisfies

$$
z_{c}=\widetilde{\alpha}_{x}+\mathcal{O}\left(\widetilde{\alpha}_{x}-\widetilde{\gamma}_{x}, \frac{\widetilde{\alpha}_{\xi}-\widetilde{\gamma}_{\xi}}{\left\langle\widetilde{\gamma}_{\xi}\right\rangle},\left(x-\gamma_{x}\right)^{2},\left(y-\alpha_{x}\right)^{2}\right)
$$

in the natural symbol sense. Notice that

$$
G\left(\gamma_{x}, \gamma, \widetilde{\gamma} ; \alpha_{x}, \alpha, \widetilde{\gamma}\right)=0 .
$$


Moreover,

$$
\begin{aligned}
d G= & \gamma_{\xi} \cdot d x-\alpha_{\xi} \cdot d y+\widetilde{\gamma}_{\xi} \cdot d \widetilde{\gamma}_{x}-\gamma_{\xi} \cdot d \gamma_{x}-\left(\widetilde{\alpha}_{\xi} \cdot d \widetilde{\alpha}_{x}-\alpha_{\xi} \cdot d \alpha_{x}\right) \\
& +\mathcal{O}\left(\left\langle\gamma_{\xi}\right\rangle\left(x-\gamma_{x}, y-\alpha_{x}, \widetilde{\gamma}_{x}-\widetilde{\alpha}_{x}, \frac{\widetilde{\gamma}_{\xi}-\widetilde{\alpha}_{\xi}}{\left\langle\gamma_{\xi}\right\rangle}\right)\right)
\end{aligned}
$$

in the same symbol sense, with the convention that the remainder term is expressed as linear combination of the "normalized" forms $d x, d y, d \alpha_{x}, \widetilde{\alpha}_{x}, d \gamma_{x}, \widetilde{\gamma}_{x}$, $\left\langle\gamma_{\xi}\right\rangle^{-1} d \alpha_{\xi}, \ldots$

Now,

$$
F_{\delta}=G_{\substack{\widetilde{\gamma}=\kappa(\gamma), \widetilde{\alpha}=\kappa(\alpha)}}+h(\alpha)-h(\gamma)
$$

and we get

$$
\begin{aligned}
d F_{\delta}= & \gamma_{\xi} \cdot d x-\alpha_{\xi} \cdot d y \\
& +\mathcal{O}\left(\left\langle\gamma_{\xi}\right\rangle\left(x-\gamma_{x}, y-\alpha_{x}, \widetilde{\gamma}_{x}-\widetilde{\alpha}_{x}, \frac{\widetilde{\gamma}_{\xi}-\widetilde{\alpha}_{\xi}}{\left\langle\gamma_{\xi}\right\rangle}\right)\right)_{\substack{\tilde{\gamma}=\kappa(\gamma) \\
\widetilde{\alpha}=\kappa(\alpha)}} .
\end{aligned}
$$

Moreover, $F_{\delta}\left(\gamma_{x}, \gamma ; \gamma_{x}, \gamma\right)=0$. From (4.45), we know that $\widetilde{\alpha}_{x}=\alpha_{x}+\widetilde{\mathcal{O}}$ $\left(\delta \epsilon^{1 / 2}\left\langle\alpha_{\xi}\right\rangle^{-1}\right), \widetilde{\alpha}_{\xi}=\alpha_{\xi}+\widetilde{\mathcal{O}}\left(\delta \epsilon^{1 / 2}\right)$ when $\widetilde{\alpha}=\kappa(\alpha)$, so

$$
\widetilde{\gamma}_{x}-\widetilde{\alpha}_{x}=\gamma_{x}-\alpha_{x}+\widetilde{\mathcal{O}}\left(\frac{\delta}{\left\langle\gamma_{\xi}\right\rangle}\right)\left(\gamma_{x}-\alpha_{x}, \frac{\gamma_{\xi}-\alpha_{\xi}}{\left\langle\gamma_{\xi}\right\rangle}\right)
$$

and similarly for $\frac{\widetilde{\gamma}_{\xi}-\widetilde{\alpha}_{\xi}}{\left\langle\alpha_{\xi}\right\rangle}$.

Hence,

$$
\begin{aligned}
d F_{\delta}= & \gamma_{\xi} \cdot d x-\alpha_{\xi} \cdot d y+\mathcal{O}\left(\left\langle\gamma_{\xi}\right\rangle\right)\left(x-\gamma_{x}, y-\alpha_{x}, \gamma_{x}-\alpha_{x}, \frac{\gamma_{\xi}-\alpha_{\xi}}{\left\langle\gamma_{\xi}\right\rangle}\right) \\
& +\widetilde{\mathcal{O}}(\delta)\left(\gamma_{x}-\alpha_{x}, \frac{\gamma_{\xi}-\alpha_{\xi}}{\left\langle\gamma_{\xi}\right\rangle}\right)
\end{aligned}
$$

and integrating this, we get

$$
\begin{aligned}
F_{\delta}(x, \gamma ; y, \alpha)= & \gamma_{\xi} \cdot\left(x-\gamma_{x}\right)-\alpha_{\xi} \cdot\left(y-\alpha_{x}\right) \\
& +\left(\mathcal{O}\left(\left\langle\gamma_{\xi}\right\rangle\right)+\widetilde{\mathcal{O}}(\delta)\right)\left(x-\gamma_{x}, y-\alpha_{x}, \gamma_{x}-\alpha_{x}, \frac{\gamma_{\xi}-\alpha_{\xi}}{\left\langle\gamma_{\xi}\right\rangle}\right)^{2}
\end{aligned}
$$

where the loss of $\epsilon^{1 / 2}$ for each differentiation appears in the variables $\alpha, \gamma$ only. When $\delta=0$, we have on the real domain

$$
\operatorname{Im} F_{\delta} \backsim\left\langle\alpha_{\xi}\right\rangle\left(\left(x-\gamma_{x}\right)^{2}+\left(y-\alpha_{x}\right)^{2}+\left(\gamma_{x}-\alpha_{x}\right)^{2}+\left(\frac{\gamma_{\xi}-\alpha_{\xi}}{\left\langle\gamma_{\xi}\right\rangle}\right)^{2}\right),
$$

and in view of (5.28) this persists for $0 \leq \delta \ll 1$. 
When applying stationary phase to (5.24) we also have to make a deformation of the integration contour in order to pass through the critical point $z_{c}$. Here we recall from [19] and (5.21) and its analogue for $\widehat{\phi}$ that

$$
\frac{1}{\left\langle\alpha_{\xi}\right\rangle} \operatorname{Im} F_{\delta} \geq \frac{1}{C}\left(\operatorname{Im} z_{c}\right)^{2}-\frac{C \delta \epsilon}{\left\langle\alpha_{\xi}\right\rangle},
$$

so the error from $\bar{\partial}_{z}$ of the almost holomorphic extension, appearing in Stokes' formula, is $\left.\mathcal{O}\left(\left(\delta \epsilon+\operatorname{I} m F_{\delta}\right) /\left\langle\alpha_{\xi}\right\rangle\right)^{\infty}\right)$. Since $\epsilon=\mathcal{O}(h)$, we conclude that

$$
\begin{aligned}
(B A)(x, y)= & \iint e^{\frac{i}{h} F_{\delta}(x, \gamma, y, \alpha)} d(x, \gamma, y, \alpha ; h) \chi\left(\left(\gamma_{x}-\alpha_{x}\right)^{2}\right. \\
& \left.+\left(\frac{\gamma_{\xi}-\alpha_{\xi}}{\left\langle\alpha_{\xi}\right\rangle}\right)^{2}\right) d \alpha d \gamma+\widehat{R},
\end{aligned}
$$

where $\widehat{R}$ has the same properties as $\widetilde{R}$ in (5.24) and

$$
\partial_{x, y}^{k} \partial_{\gamma_{x}, \alpha_{x}}^{\ell} \partial_{\gamma_{\xi}, \alpha_{\xi}}^{p} d=\mathcal{O}\left(h^{-m-\widehat{m}-3 n+\frac{n}{2}}\left\langle\alpha_{\xi}\right\rangle^{k+\widehat{k}+\frac{n}{2}-|p|} \epsilon^{-\frac{|\ell+p|}{2}}\right) .
$$

We now compute the Weyl symbol $c$ of $B A$ by means of the formula

$$
c(x, \xi ; h)=\int(B A)\left(x+\frac{w}{2}, x-\frac{w}{2}\right) e^{-i w \xi / h} d w .
$$

The contribution from $\widehat{R}$ in $(5.30)$ is $\mathcal{O}\left(h^{\infty}\langle\xi\rangle^{-\infty}\right)$ with all its derivatives. The contribution from the integral in $(5.30)$ is

$$
\begin{array}{r}
\iiint e^{\frac{i}{h}\left(F_{\delta}\left(x+\frac{w}{2}, \gamma, x-\frac{w}{2}, \alpha\right)-w \cdot \xi\right)} d\left(x+\frac{w}{2}, \gamma, x-\frac{w}{2}, \alpha ; h\right) \chi\left(\left(\gamma_{x}-\alpha_{x}\right)^{2}\right. \\
\left.+\left(\frac{\gamma_{\xi}-\alpha_{\xi}}{\left\langle\alpha_{\xi}\right\rangle}\right)^{2}\right) d w d \alpha d \gamma .
\end{array}
$$

The contribution from a region $\left\{\left|\xi-\alpha_{\xi}\right| \geq \frac{1}{C}\left\langle\alpha_{\xi}\right\rangle\right\}$ is $\mathcal{O}\left(h^{\infty}\langle\xi\rangle^{-\infty}\right)$ with all its derivatives and the remaining region can be treated with the method of stationary phase by working in the dilated tilde variables given by $\gamma_{x}=x_{0}+\sqrt{\epsilon} \widetilde{\gamma}_{x}, \ldots$ as in the addendum below. The proposition follows.

Addendum. Stationary phase with $\widetilde{\mathcal{O}}$-symbols. Assume

$$
\begin{gathered}
\phi \in C^{\infty}\left(\mathbf{R}^{n}\right), \quad \operatorname{Im} \phi \geq 0, \quad \phi(0)=0, \\
\left|\phi^{\prime}(x)\right| \backsim|x|, \quad \phi^{\prime}=x \cdot \widetilde{\mathcal{O}}(1), \quad \operatorname{det} \phi^{\prime \prime}(0) \neq 0 \text { uniformly in } \epsilon .
\end{gathered}
$$

Let $a=\widetilde{\mathcal{O}}(1)$. We shall establish a stationary phase development for

$$
I(h)=h^{-\frac{n}{2}} \int e^{\frac{i}{h} \phi(x)} a(x) d x
$$

in powers of $\widetilde{h}=h / \epsilon$. (Assume $h \ll \epsilon \ll 1$.) 
Put $x=\epsilon^{\frac{1}{2}} \widetilde{x}$,

$$
I(h)=\left(\frac{h}{\epsilon}\right)^{-\frac{n}{2}} \int e^{\frac{i}{h} \widetilde{\phi}(\widetilde{x})} \widetilde{a}(\widetilde{x}) d \widetilde{x},
$$

where $\phi\left(\epsilon^{\frac{1}{2}} \widetilde{x}\right)=\epsilon \widetilde{\phi}(\widetilde{x}), \widetilde{\phi}(\widetilde{x}) \equiv \epsilon \phi\left(\epsilon^{\frac{1}{2}} \widetilde{x}\right), \widetilde{a}(\widetilde{x})=a\left(\epsilon^{\frac{1}{2}} \widetilde{x}\right)$.

Then $\widetilde{a}=\mathcal{O}(1)$ in the symbol sense; $\partial^{\alpha} \widetilde{a}=\mathcal{O}(1)$, and

$$
\left|\widetilde{\phi^{\prime}}(\widetilde{x})\right|=\left|\frac{1}{\sqrt{\epsilon}} \phi^{\prime}(\sqrt{\epsilon} \widetilde{x})\right| \backsim|\widetilde{x}|, \quad \widetilde{\phi}^{\prime}(\widetilde{x})=\widetilde{x} \cdot \mathcal{O}(1)
$$

and $\widetilde{\phi}^{\prime \prime}(0)=\phi^{\prime \prime}(0)$. The contribution to $I(h)$ from $|\widetilde{x}| \geq 1$ is $\mathcal{O}\left(\widetilde{h}^{\infty}\right)$ by repeated integrations by parts, and the contribution from $|\widetilde{x}|<1$ can be handled in the usual way since $\widetilde{\phi}=\mathcal{O}(1)$ here. Thus

$$
I(h) \sim \sum_{0}^{\infty} c_{j} \widetilde{h}^{j}, \quad c_{0}=\frac{(2 \pi)^{n / 2}}{\sqrt{\operatorname{det} \phi^{\prime \prime}(0)}} e^{i \frac{\pi}{4} \operatorname{sgn} \phi^{\prime \prime}(0)} .
$$

It follows from the proof that the proposition remains valid if we relax the symbol condition in $y$ and only assume

$$
\partial_{x}^{\ell} \partial_{\alpha_{x}, y}^{q} \partial_{\alpha_{\xi}}^{p} a=\mathcal{O}\left(h^{-\frac{3 n}{2}-m}\left\langle\alpha_{\xi}\right\rangle^{\frac{n}{2}+k-|p|} \epsilon^{-\frac{1}{2}(|q|+|p|)}\right),
$$

i.e., we also allow for a loss of $\epsilon^{1 / 2}$ for each $y$-derivation. Similarly for $B$ (cf. (5.18)) we can content ourselves with

$$
\partial_{y}^{\ell} \partial_{\gamma_{x}, x}^{q} \partial_{\gamma_{\xi}}^{p} b=\mathcal{O}\left(h^{-\frac{3 n}{2}-\widehat{m}}\left\langle\alpha_{\xi}\right\rangle^{\frac{n}{2}+\widehat{k}-|p|} \epsilon^{-\frac{1}{2}(|q|+|p|)}\right) .
$$

Moreover, if $b$ and $a$ are elliptic, then $c$ is elliptic.

We get by standard arguments,

Proposition 5.4. Let $A$ be an elliptic Fourier integral operator of order $(m, k)$ with symbol as in (5.35). Then there exists an elliptic Fourier integral operator $B$ of order $(-m,-k)$ with symbol as in $(5.36)$, such that

$$
B A=1+R,
$$

where $R$ is 1-negligible in the sense that its symbol $R$ is $\widetilde{\mathcal{O}}\left(\left(\frac{h}{\epsilon}\right)^{\infty}\langle\xi\rangle^{-\infty}\right)$. In particular $A$ has the left inverse $(1+R)^{-1} B$ when $\epsilon / h \gg 1$.

We notice that when $\delta=0$, then $A, B$ are elliptic pseudodifferential operators and hence $(1+R)^{-1} B$ is also a left inverse. (By the Beals lemma we also know that $(1+R)^{-1}$ is an $h$-pseudodifferential operator with symbol $1+\widetilde{\mathcal{O}}\left(\left(\frac{h}{\epsilon\langle\xi\rangle}\right)^{\infty}\right)$.) For general small $\delta, \mathcal{R}\left(A_{\delta}\right)$ is closed. Using suitable deformations of $A$ we can produce a continuous deformation of closed subspaces in $L^{2}$ from $L^{2}$ to $\mathcal{R}\left(A_{\delta}\right)$. All the deformed subspaces then have to be equal to $L^{2}$ and $\mathcal{R}\left(A_{\delta}\right)=L^{2}$, so $\left(1+R_{\delta}\right)^{-1} B_{\delta}$ is also a left inverse of $A_{\delta}$. 
We next turn to Egorov's theorem and start with some preparations. Recall that by (4.45)

$$
\kappa(\alpha)_{x}=\alpha_{x}+\widetilde{\mathcal{O}}\left(\delta \epsilon^{\frac{1}{2}}\left\langle\alpha_{\xi}\right\rangle^{-1}\right), \quad \kappa(\alpha)_{\xi}=\alpha_{\xi}+\widetilde{\mathcal{O}}\left(\delta \epsilon^{\frac{1}{2}}\right) .
$$

It follows that

$$
\begin{aligned}
& d_{\alpha}\left(\kappa(\alpha)_{\xi}\right)=d \alpha_{\xi}+\widetilde{\mathcal{O}}(\delta) d \alpha_{x}+\widetilde{\mathcal{O}}\left(\frac{\delta}{\left\langle\alpha_{\xi}\right\rangle}\right) d \alpha_{\xi} \\
& d_{\alpha}\left(\kappa(\alpha)_{x}\right)=d \alpha_{x}+\widetilde{\mathcal{O}}\left(\frac{\delta}{\left\langle\alpha_{\xi}\right\rangle}\right) d \alpha_{x}+\widetilde{\mathcal{O}}\left(\frac{\delta}{\left\langle\alpha_{\xi}\right\rangle^{2}}\right) d \alpha_{\xi} .
\end{aligned}
$$

Substituting this into (5.17), we get

$$
\begin{aligned}
d_{\alpha} \phi= & \left(\left(x-\kappa(\alpha)_{x}\right)-\left(y-\alpha_{x}\right)\right) \cdot d \alpha_{\xi} \\
& -\left(\frac{\partial \psi}{\partial x}+\frac{\partial \psi}{\partial y}\right)\left(x-\kappa(\alpha)_{x}, y-\alpha_{x}, \alpha_{\xi}\right) \cdot d \alpha_{x} \\
& +\left(x-\kappa(\alpha)_{x}, y-\alpha_{x}\right) \cdot\left(\widetilde{\mathcal{O}}(\delta) d \alpha_{x}+\widetilde{\mathcal{O}}\left(\frac{\delta}{\left\langle\alpha_{\xi}\right\rangle}\right) d \alpha_{\xi}\right) \\
& +\frac{\partial \psi}{\partial \alpha}\left(x-\kappa(\alpha)_{x}, y-\alpha_{x}, \alpha\right) \cdot d \alpha,
\end{aligned}
$$

which we can write

$$
\begin{aligned}
\left(\begin{array}{c}
\partial_{\alpha_{x}} \phi /\left\langle\alpha_{\xi}\right\rangle \\
\partial_{\alpha_{\xi}} \phi
\end{array}\right)= & \left(\begin{array}{c}
\left(\frac{\partial \psi}{\partial \alpha_{x}}-\left(\frac{\partial \psi}{\partial x}+\frac{\partial \psi}{\partial y}\right)\right) /\left\langle\alpha_{\xi}\right\rangle \\
\left(x-\kappa(\alpha)_{x}\right)-\left(y-\alpha_{x}\right)+\frac{\partial \psi}{\partial \alpha_{\xi}}
\end{array}\right) \\
& +\widetilde{\mathcal{O}}\left(\frac{\delta}{\left\langle\alpha_{\xi}\right\rangle}\right)\left(\begin{array}{c}
x-\kappa(\alpha)_{x} \\
y-\alpha_{x}
\end{array}\right) .
\end{aligned}
$$

Here we write $a(x, y, \alpha)=\widetilde{\mathcal{O}}(m)$ if $\partial_{x, y}^{\nu} \partial_{\alpha_{x}}^{\mu} \partial_{\alpha_{\xi}}^{\rho} a=\mathcal{O}\left(m \epsilon^{-\frac{1}{2}(|\mu|+|\rho|)}\left\langle\alpha_{\xi}\right\rangle^{-|\rho|}\right)$. The differential of this vector at a point of the critical set is given by the matrix

$$
\left(\begin{array}{cc}
-\left\langle\alpha_{\xi}\right\rangle^{-1}\left(\psi_{x x}^{\prime \prime}+\psi_{y x}^{\prime \prime}\right) & -\left\langle\alpha_{\xi}\right\rangle^{-1}\left(\psi_{x y}^{\prime \prime}+\psi_{y y}^{\prime \prime}\right) \\
1 & -1
\end{array}\right)+\widetilde{\mathcal{O}}\left(\frac{\delta}{\left\langle\alpha_{\xi}\right\rangle}\right) .
$$

If $\left(\begin{array}{c}t_{x} \\ t_{y}\end{array}\right)$ is in the kernel of the first term, we get $t_{x}=t_{y}$ and $\left(\psi_{x x}^{\prime \prime}+\psi_{y x}^{\prime \prime}+\psi_{x y}^{\prime \prime}+\right.$ $\left.\psi_{y y}^{\prime \prime}\right) t_{x}=0$. Here we recognize the Hessian of $z \mapsto \psi(z, z, \alpha)$ which is invertible because of the assumption on the imaginary part. More precisely, the matrix (5.42) has a uniformly bounded inverse. From (5.41), we get

$$
\left.\begin{array}{rl}
\left(x-\kappa(\alpha)_{x}\right. \\
y-\alpha_{x}
\end{array}\right)=M_{\delta}(x, y, \alpha)\left(\begin{array}{c}
\partial_{\alpha_{x}} \phi /\left\langle\alpha_{\xi}\right\rangle \\
\partial_{\alpha_{\xi}} \phi
\end{array}\right),
$$

where

and $\partial_{x, y, \alpha_{x}}^{k} \partial_{\alpha_{\xi}}^{\ell} M_{0}=\mathcal{O}\left(\left\langle\alpha_{\xi}\right\rangle^{-|\ell|}\right)$.

$$
M_{\delta}(x, y, \alpha)=M_{0}(x, y, \alpha)+\widetilde{\mathcal{O}}\left(\frac{\delta}{\left\langle\alpha_{\xi}\right\rangle}\right),
$$


Lemma 5.5. Let $\Phi(x, y, \alpha)=\widetilde{\mathcal{O}}\left(\left\langle\alpha_{\xi}\right\rangle^{\widetilde{m}}\right)$ in the sense that

$$
\partial_{x}^{k} \partial_{y, \alpha_{x}}^{\ell} \partial_{\alpha_{\xi}}^{p} \Phi=\mathcal{O}\left(\epsilon^{-\frac{1}{2}(|\ell|+|p|)}\left\langle\alpha_{\xi}\right\rangle^{\widetilde{m}-|p|}\right) .
$$

Similarly, let $a=\widetilde{\mathcal{O}}\left(\left\langle\alpha_{\xi}\right\rangle^{\widetilde{n}}\right)$ be elliptic. Then

$$
\begin{aligned}
\iint e^{\frac{i}{h} \phi} \Phi(x, y, \alpha) a(x, y, \alpha ; h) \chi(x, y, \alpha) u(y) d y d \alpha \\
=\iint e^{\frac{i}{h} \phi} \Phi\left(\kappa(\alpha)_{x}, \alpha_{x}, \alpha\right) a(x, y, \alpha ; h) \chi(x, y, \alpha) u(y) d y d \alpha \\
\quad+\iint e^{\frac{i}{h} \phi} \widetilde{\mathcal{O}}\left(\frac{h}{\epsilon}\left\langle\alpha_{\xi}\right\rangle^{\widetilde{m}-1}\right) a(x, y, \alpha ; h) \widetilde{\chi}(x, y, \alpha) u(y) d y d \alpha,
\end{aligned}
$$

where $\tilde{\chi}$ is a similar cutoff.

Proof. We have

$$
\begin{aligned}
\Phi(x, y, \alpha) & =\Phi\left(\kappa(\alpha)_{x}, \alpha_{x}, \alpha\right)+\widetilde{\mathcal{O}}\left(\epsilon^{-\frac{1}{2}}\left\langle\alpha_{\xi}\right\rangle^{\widetilde{m}}\right)\left(\begin{array}{c}
x-\kappa(\alpha)_{x} \\
y-\alpha_{x}
\end{array}\right) \\
& =\Phi\left(\kappa(\alpha)_{x}, \alpha_{x}, \alpha\right)+\widetilde{\mathcal{O}}\left(\epsilon^{-\frac{1}{2}}\left\langle\alpha_{\xi}\right\rangle^{\widetilde{m}-1}\right) \cdot \partial_{\alpha_{x}} \phi+\widetilde{\mathcal{O}}\left(\epsilon^{-\frac{1}{2}}\left\langle\alpha_{\xi}\right\rangle^{\widetilde{m}}\right) \cdot \partial_{\alpha_{\xi}} \phi .
\end{aligned}
$$

The contribution from the remainders to the left hand side in (5.45) is therefore

$$
\begin{aligned}
h \iint \frac{\partial}{\partial \alpha_{x}}\left(e^{\frac{i}{h} \phi}\right) \cdot \widetilde{\mathcal{O}}\left(\epsilon^{-\frac{1}{2}}\left\langle\alpha_{\xi}\right\rangle^{\widetilde{m}-1}\right) & a \chi u d y d \alpha \\
& +h \iint \frac{\partial}{\partial \alpha_{\xi}}\left(e^{\frac{i}{h} \phi}\right) \cdot \widetilde{\mathcal{O}}\left(\epsilon^{-\frac{1}{2}}\left\langle\alpha_{\xi}\right\rangle^{\widetilde{m}}\right) a \chi u d y d \alpha,
\end{aligned}
$$

and it suffices to integrate by parts.

Actually, we shall not use the lemma directly, only its proof. The next result is closely related and could probably be obtained from Lemma 5.5. We will give a different proof however.

Lemma 5.6. Under the same assumptions as in the preceding lemma, we have

$$
\iint e^{\frac{i}{h} \phi} \Phi a \chi u(y) d y d \alpha=A Q,
$$

where $Q$ is an h-pseudodifferential operator with symbol

$$
Q=\Phi\left(\kappa(x, \xi)_{x}, x, x, \xi\right)+\widetilde{\mathcal{O}}\left(\frac{h}{\epsilon}\langle\xi\rangle^{\widetilde{m}-1}\right) .
$$

Here

$$
A u(x)=\iint e^{\frac{i}{h} \phi} a \chi u(y) d y d \alpha .
$$

Proof. In order to harmonize with Proposition 5.3, we may change the assumption on $a$ to $a=\widetilde{\mathcal{O}}\left(\left\langle\alpha_{\xi}\right\rangle^{\widetilde{m}+\frac{n}{2}} h^{-\frac{3 n}{2}}\right)$ and assume that $a$ is elliptic in this class. Here $\widetilde{\mathcal{O}}$ indicates a loss of $\epsilon^{1 / 2}$ for each differentiation in $\alpha, y$. Let $B$ be of the form (5.18) with $b=\widetilde{\mathcal{O}}\left(\left\langle\alpha_{\xi}\right\rangle^{-\widetilde{m}+\frac{n}{2}} h^{-\frac{3 n}{2}}\right)$ where the $\epsilon^{1 / 2}$ loss is now for each differentiation 
in $(x, \alpha)$. We also assume that $b$ is elliptic. Then from Proposition 5.3 and the remark following its proof, we know that $B A=\mathrm{Op}_{h}(c)$, where $c=\widetilde{\mathcal{O}}(1)$ is elliptic. Recalling that the proof was by stationary phase, we see that if $\widetilde{A}$ is the Operator given by the left hand side of (5.46), then $B \widetilde{A}=\mathrm{Op}_{h}(\widetilde{c})$, where

$$
\widetilde{c}=\Phi(\kappa(x, \xi), x, x, \xi) c+\widetilde{\mathcal{O}}\left(\frac{h}{\epsilon}\langle\xi\rangle^{\widetilde{m}-1}\right) .
$$

By pseudodifferential calculus, we get $B \widetilde{A}=B A Q$, with $Q$ as in the lemma. Moreover, $B$ is invertible.

Remark 5.7. Later on, we shall meet the special situation when $\Phi=\Phi(\alpha)=$ $\widetilde{\mathcal{O}}\left(\left\langle\alpha_{\xi}\right\rangle^{m_{1}}\right) P(\alpha)=: R P, P(\alpha)=\mathcal{O}\left(\langle\xi\rangle^{m_{2}}\right)$. In this case we have

$$
Q=\widetilde{\mathcal{O}}\left(\langle\xi\rangle^{m_{1}}\right) \# P+\widetilde{\mathcal{O}}\left(\frac{h}{\sqrt{\epsilon}}\langle\xi\rangle^{m_{1}+m_{2}-1}\right) .
$$

In fact (anticipating on a part of the proof of Proposition 5.9), let

$$
B u=\iint e^{\frac{i}{h} \phi} \operatorname{Ra\chi } u(y) d y d \alpha .
$$

Then (with $\mathrm{Op}=\mathrm{Op}_{h}$ when nothing else is specified)

$$
\begin{aligned}
B \circ \mathrm{Op}(P) u & =\iint{ }^{t} P\left(y, h D_{y}\right)\left(e^{\frac{i}{h} \phi} \operatorname{Ra\chi }\right) u(y) d y d \alpha \\
& =\iint\left(P\left(y,-\phi_{y}^{\prime}\right) R a \chi+\widetilde{\mathcal{O}}\left(h^{2}\left\langle\alpha_{\xi}\right\rangle^{m_{1}+m_{2}-2}\right)\right) e^{\frac{i}{h} \phi} u(y) d y d \alpha .
\end{aligned}
$$

As we shall see in the proof of Proposition 5.9,

$$
\begin{aligned}
\iint P\left(y,-\phi_{y}^{\prime}\right) \operatorname{Ra\chi } e^{\frac{i}{h} \phi} u(y) d y d \alpha= & \iint e^{\frac{i}{h} \phi} \Phi a \chi u(y) d y d \alpha \\
& +\iint e^{\frac{i}{h} \phi} \widetilde{\mathcal{O}}\left(\frac{h}{\sqrt{\epsilon}}\left\langle\alpha_{\xi}\right\rangle^{m_{1}+m_{2}-1}\right) u(y) d y d \alpha .
\end{aligned}
$$

Applying Lemma 5.6 to $B$ and the various remainder terms, we get (5.49).

Proposition 5.8. Let $A_{s}$ be a Fourier integral operator quantizing $\kappa(s)$ as in Proposition 5.3. Also, assume that $a=a_{s}$ is elliptic and depends smoothly on $s$ in the sense that

$$
\partial_{s}^{k} a_{s}=\widetilde{\mathcal{O}}\left(h^{-m-\frac{3 n}{2}}\left\langle\alpha_{\xi}\right\rangle^{k+\frac{n}{2}}\right),
$$

where $\widetilde{\mathcal{O}}$ indicates a loss of $\epsilon^{1 / 2}$ for differentiations in $\alpha, y$. Then

$$
h D_{s} A_{s}+i A_{s} \check{\psi}_{s}=0, \quad 0 \leq s \leq \delta_{0}, \quad 0<\delta_{0} \ll 1,
$$

where $D_{s}=i^{-1} \partial_{s}$ and $\check{\psi}_{s}$ is an h-pseudodifferential operator with symbol

$$
\check{\psi}_{s}=\widetilde{\mathcal{O}}\left(h+h\langle\xi\rangle^{-1}\right)+\widehat{\psi}_{\epsilon, s} \circ \kappa(s) .
$$

The term " $h$ " in the remainder can be dropped if $a_{s}$ is independent of $s$. 
Proof. Assume for simplicity that $a_{s}$ is independent of $s$. Then,

$$
h D_{s} A_{s} u(x)=\iint e^{\frac{i}{h} \phi} a \partial_{s} \phi u d y d \alpha .
$$

Recalling that,

$$
\begin{aligned}
\phi_{s}(x, y, \alpha)= & \left(x-\kappa(s, \alpha)_{x}\right) \cdot \kappa(s, \alpha)_{\xi}+\left(\alpha_{x}-y\right) \cdot \alpha_{\xi} \\
& +\psi\left(x-\kappa(s, \alpha)_{x}, y-\alpha_{x}, \alpha\right)+h_{s}(\alpha),
\end{aligned}
$$

we get

$$
\begin{aligned}
\partial_{s} \phi_{s}(x, y, \alpha)= & \left(x-\kappa(s, \alpha)_{x}\right) \cdot \partial_{s} \kappa(s, \alpha)_{\xi}-\partial_{s} \kappa(s, \alpha)_{x} \cdot \kappa(s, \alpha)_{\xi} \\
& -\left(\partial_{x} \psi\right)\left(x-\kappa(s, \alpha)_{x}, y-\alpha_{x}, \alpha\right) \cdot \partial_{s} \kappa(s, \alpha)_{x}+\partial_{s} h_{s}(\alpha) .
\end{aligned}
$$

The restriction to the critical set (5.16) is

$$
\begin{aligned}
\left(\partial_{s} \phi_{s}\right)\left(\kappa(s, \alpha)_{x}, \alpha_{x}, \alpha\right) & =\left(\partial_{s} h_{s}\right)(\alpha)-\partial_{s} \kappa(s, \alpha)_{x} \cdot \kappa(s, \alpha)_{\xi} \\
& =\left(\partial_{s} h_{s}\right)(\alpha)-i\left(\frac{\partial}{\partial \xi} \widehat{\psi}_{\epsilon, s} \cdot \xi\right) \circ \kappa(s) \\
& =-i \widehat{\psi}_{\epsilon, s} \circ \kappa(s)(\alpha) \\
& =\widetilde{\mathcal{O}}(\epsilon),
\end{aligned}
$$

where we used (5.3). More generally, from (5.53) we get

$$
\partial_{s} \phi_{s}=-i \widehat{\psi}_{\epsilon, s} \circ \kappa(s)(\alpha)+\widetilde{\mathcal{O}}\left(\epsilon^{\frac{1}{2}}\right) \cdot\left(x-\kappa(s, \alpha)_{x}, y-\alpha_{x}\right) .
$$

As in the proof of Lemma (5.5) we can make integrations by parts and see that the contribution from the remainder to (5.52) becomes

$$
\iint e^{\frac{i}{h} \phi} \widetilde{\mathcal{O}}\left(h^{-m-\frac{3 n}{2}}\left\langle\alpha_{\xi}\right\rangle^{k+\frac{n}{2}} h\left\langle\alpha_{\xi}\right\rangle^{-1}\right) u(y) d y d \alpha .
$$

Combining this with (5.55) and Lemma 5.6, we get the proposition.

We can now prove Proposition 5.2.

Proof. We only consider $A$ and we may assume that $A=A(\delta)$ where $A(s)$ is a smooth family as in the preceding proposition. The result for $B$ will be the same since $B$ is like the adjoint of $A$. From (5.50) we get

$$
h \partial_{s} A_{s}^{*}=\check{\psi}_{s}^{*} A_{s}^{*} .
$$

If $u \in \mathcal{S}$, then $A_{s}^{*} u \in \mathcal{S}$ and we get

$$
h \partial_{s}\left\|A_{s}^{*} u\right\|^{2}=\left(\check{\psi}_{s}^{*} A_{s}^{*} u \mid A_{s}^{*} u\right)+\left(A_{s}^{*} u \mid \check{\psi}_{s}^{*} A_{s}^{*} u\right),
$$

SO

But $\left\|\check{\psi}_{s}^{*}\right\|=\mathcal{O}(\epsilon)$, so

$$
h \partial_{s}\left\|A_{s}^{*} u\right\|^{2} \leq 2\left\|\check{\psi}_{s}^{*}\right\|\left\|A_{s}^{*} u\right\|^{2} .
$$

$$
\left\|A_{s}^{*} u\right\| \leq e^{\mathcal{O}(\epsilon)|s| / h}\|u\|=e^{\mathcal{O}(1) A|s|}\|u\| \quad(\epsilon=A h) .
$$


Then $\left\|A_{\delta}^{*}\right\| \leq e^{\mathcal{O}(1) A \delta}$ and the proposition follows since $A_{\delta}$ and $A_{\delta}^{*}$ have the same norm.

Let $P=\mathcal{O}\left(\langle\xi\rangle^{M}\right)$ in the symbol sense: $\partial_{x}^{\alpha} \partial_{\xi}^{\beta} P=\mathcal{O}\left(\langle\xi\rangle^{M-|\beta|}\right)$. We study $P A_{s}-A_{s} P$ where $A_{s}$ is as in Proposition 5.8, $\partial_{s}^{\ell} a_{s}$ bounded in $S^{m+\frac{3 n}{2}, k+\frac{n}{2}}$ (so no $\sqrt{\epsilon}$-loss in the symbol for simplicity). Working with the Weyl quantization we know that

$$
\begin{aligned}
& P\left(x, h D_{x} ; h\right)\left(e^{\frac{i}{h} \phi(x, y, \alpha)} a(x, y, \alpha ; h)\right)=e^{\frac{i}{h} \phi} b+\widetilde{\mathcal{O}}\left(h^{\infty}\left\langle\alpha_{\xi}\right\rangle^{-\infty}\right) \\
& { }^{t} P\left(y, h D_{y} ; h\right)\left(e^{\frac{i}{h} \phi(x, y, \alpha)} a(x, y, \alpha ; h)\right)=e^{\frac{i}{h} \phi} c+\widetilde{\mathcal{O}}\left(h^{\infty}\left\langle\alpha_{\xi}\right\rangle^{-\infty}\right),
\end{aligned}
$$

where

$$
\begin{aligned}
& b \equiv P\left(x, \phi_{x}^{\prime}(x, y, \alpha) ; h\right) a(x, y, \alpha ; h)+\widetilde{\mathcal{O}}\left(h^{-\frac{3 n}{2}-m}\left\langle\alpha_{\xi}\right\rangle^{k+\frac{n}{2}+M} h^{2}\left\langle\alpha_{\xi}\right\rangle^{-2}\right), \\
& c \equiv P\left(y,-\phi_{y}^{\prime}(x, y, \alpha) ; h\right) a(x, y, \alpha ; h)+\widetilde{\mathcal{O}}\left(h^{-\frac{3 n}{2}-m}\left\langle\alpha_{\xi}\right\rangle^{k+\frac{n}{2}+M} h^{2}\left\langle\alpha_{\xi}\right\rangle^{-2}\right),
\end{aligned}
$$

and the $\widetilde{\mathcal{O}}$ refers to $\sqrt{\epsilon}$-loss only with respect to differentiations in $\alpha$. (Recall the general fact that the Weyl symbol of $e^{-i \phi / h} \circ p^{w}\left(x, h D_{x}\right) \circ e^{i \phi / h}$ is equal to $\left.p\left(x, \xi+\phi_{x}^{\prime}\right)+\mathcal{O}\left(h^{2}\right).\right)$

We conclude that $P A_{s}-A_{s} P=\widetilde{A}_{s}+A_{s} \circ \mathrm{Op}\left(\widetilde{\mathcal{O}}\left(h^{2}\left\langle\alpha_{\xi}\right\rangle^{-2}\right)\right)$, where

$$
\widetilde{A}_{s} u=\iint e^{\frac{i}{h} \phi}\left(P\left(x, \phi_{x}^{\prime}(x, y, \alpha) ; h\right)-P\left(y,-\phi_{y}^{\prime} ; h\right)\right) a u(y) d y d \alpha .
$$

On the critical set (5.16), we have

$$
P\left(x, \phi_{x}^{\prime} ; h\right)-P\left(y,-\phi_{y}^{\prime} ; h\right)=P(\kappa(s)(\alpha))-P(\alpha),
$$

and more generally,

$$
\begin{aligned}
P\left(x, \phi_{x}^{\prime}\right)-P\left(y,-\phi_{y}^{\prime}\right)= & P(\kappa(s)(\alpha))-P(\alpha) \\
& +\left(x-\kappa(s, \alpha)_{x}\right) \cdot\left(\mathcal{O}\left(\left\langle\alpha_{\xi}\right\rangle^{M}\right)+\widetilde{\mathcal{O}}\left(s \epsilon^{\frac{1}{2}}\left\langle\alpha_{\xi}\right\rangle^{M-1}\right)\right) \\
& +\left(y-\alpha_{x}\right) \cdot\left(\mathcal{O}\left(\left\langle\alpha_{\xi}\right\rangle^{M}\right)+\widetilde{\mathcal{O}}\left(s \epsilon^{\frac{1}{2}}\left\langle\alpha_{\xi}\right\rangle^{M-1}\right)\right) .
\end{aligned}
$$

Using (5.43), (5.44), we get

$$
\begin{aligned}
P\left(x, \phi_{x}^{\prime}\right)-P\left(y,-\phi_{y}^{\prime}\right)= & P(\kappa(s, \alpha))-P(\alpha) \\
& +\left(\phi_{\alpha_{\xi}}^{\prime},\left\langle\alpha_{\xi}\right\rangle^{-1} \phi_{\alpha_{x}}^{\prime}\right) \cdot\left(\mathcal{O}\left(\left\langle\alpha_{\xi}\right\rangle^{M}\right)+\widetilde{\mathcal{O}}\left(s \epsilon^{\frac{1}{2}}\left\langle\alpha_{\xi}\right\rangle^{M-1}\right)\right) .
\end{aligned}
$$

The contribution from $P(\kappa(s)(\alpha))-P(\alpha)$ in (5.56) is of the form

$$
A_{s} \circ \mathrm{Op}\left(P(\kappa(s)(\alpha))-P(\alpha)+\widetilde{\mathcal{O}}\left(s \frac{h}{\sqrt{\epsilon}}\left\langle\alpha_{\xi}\right\rangle^{M-2}\right)\right)
$$

in general, and the remainder estimate improves to $\widetilde{\mathcal{O}}\left(\operatorname{sh}\left\langle\alpha_{\xi}\right\rangle^{M-2}\right)$ if $P(\kappa(s, \alpha))-$ $P(\alpha)=\widetilde{\mathcal{O}}\left(s \epsilon\langle\alpha\rangle^{M-1}\right)$. By integration by parts in $\alpha$, we see that the contribution 
from the two remainder terms in (5.57) is

$$
\begin{gathered}
h \iint e^{\frac{i}{h} \phi} \partial_{\alpha_{\xi}}\left(\mathcal{O}\left(\left\langle\alpha_{\xi}\right\rangle^{M}\right)+\widetilde{\mathcal{O}}\left(s \epsilon^{\frac{1}{2}}\left\langle\alpha_{\xi}\right\rangle^{M-1}\right)+\partial_{\alpha_{x}}\left(\frac{1}{\left\langle\alpha_{\xi}\right\rangle}(\text { idem })\right) a\right) u(y) d y d \alpha \\
=h \iint e^{\frac{i}{h} \phi}\left(\mathcal{O}\left(\left\langle\alpha_{\xi}\right\rangle^{M-1}\right)+\widetilde{\mathcal{O}}\left(s\left\langle\alpha_{\xi}\right\rangle^{M-2}\right)\right) a u(y) d y d \alpha .
\end{gathered}
$$

In conclusion,

Proposition 5.9. We have

$$
P A_{s}=A_{s} P_{s}, \quad P_{s}=P+Q_{s},
$$

where

$$
Q_{s}=P(\kappa(s)(\alpha))-P(\alpha)+\widetilde{\mathcal{O}}\left(s \frac{h}{\epsilon^{\frac{1}{2}}}\left\langle\alpha_{\xi}\right\rangle^{M-2}\right)+\widetilde{\mathcal{O}}\left(h\left\langle\alpha_{\xi}\right\rangle^{M-1}\right)
$$

in the general case, when $P(\kappa(s)(\alpha))-P(\alpha)=\widetilde{\mathcal{O}}\left(s \epsilon^{\frac{1}{2}}\left\langle\alpha_{\xi}\right\rangle^{M-1}\right)$.

In the special case when $P(\kappa(s, \alpha))-P(\alpha)=\widetilde{\mathcal{O}}\left(s \epsilon\left\langle\alpha_{\xi}\right\rangle^{M-1}\right)$ the first remainder term improves to $\widetilde{\mathcal{O}}\left(\operatorname{sh}\left\langle\alpha_{\xi}\right\rangle^{M-2}\right)$ so in that case,

$$
Q_{s}(\alpha)=P(\kappa(s, \alpha))-P(\alpha)+\widetilde{\mathcal{O}}\left(h\left\langle\alpha_{\xi}\right\rangle^{M-1}\right) .
$$

In order to treat certain conjugations, we need a more precise description of $Q_{s}$ in the general case in the last proposition. The proof above shows that

$$
A_{s} Q_{s} u=\iint e^{\frac{i}{h} \phi}(P(\kappa(s, \alpha))-P(\alpha)) a u(y) d y d \alpha+A_{s} \mathrm{Op}\left(\widetilde{\mathcal{O}}\left(h\langle\xi\rangle^{M-1}\right)\right),
$$

and we need to take a closer look at the oscillatory integral.

By Taylor's formula and (4.45),

$$
P(\kappa(\alpha))-P(\alpha)=\widetilde{\mathcal{O}}(s \sqrt{\epsilon}) \cdot\left(P_{\alpha_{\xi}}^{\prime}(\alpha), \frac{1}{\left\langle\alpha_{\xi}\right\rangle} P_{\alpha_{x}}^{\prime}(\alpha)\right)+\widetilde{\mathcal{O}}\left(s^{2} \epsilon\left\langle\alpha_{\xi}\right\rangle^{M-2}\right) .
$$

When passing to Weyl composition of symbols, we notice that if $r=\widetilde{\mathcal{O}}(s \sqrt{\epsilon})$, then

$$
r \#\left(P_{\alpha_{\xi}}^{\prime}, \frac{1}{\left\langle\alpha_{\xi}\right\rangle} P_{\alpha_{x}}^{\prime}\right)=r \cdot\left(P_{\alpha_{\xi}}^{\prime}, \frac{1}{\left\langle\alpha_{\xi}\right\rangle} P_{\alpha_{x}}^{\prime}\right)+\widetilde{\mathcal{O}}\left(\operatorname{sh}\left\langle\alpha_{\xi}\right\rangle^{M-2}\right) .
$$

Lemma 5.6 and Remark 5.7 then show that on the symbol level

$$
Q_{s}=\widetilde{\mathcal{O}}\left(h\langle\xi\rangle^{M-1}\right)+\widetilde{\mathcal{O}}\left(s^{2} \epsilon\langle\xi\rangle^{M-2}\right)+\widetilde{\mathcal{O}}(s \sqrt{\epsilon}) \#\left(P_{\xi}^{\prime}, \frac{1}{\langle\xi\rangle} P_{x}^{\prime}\right) .
$$

Here the first term to the right is too large; we would like to have $h\langle\xi\rangle^{M-2}$, so we take a closer look at $Q_{s}$ using Proposition 5.8, where we now add the assumption

$$
m=k=0, \quad A_{0}=1, \quad a_{s} \text { is independent of } s .
$$

Then as noticed, (5.51) improves to

$$
\check{\psi}_{s}=\widehat{\psi}_{s} \circ \kappa(s)+\widetilde{\mathcal{O}}\left(\frac{h}{\langle\xi\rangle}\right)=\widetilde{\mathcal{O}}(\epsilon) .
$$


We get on the symbol level, writing $[A, B]=A \# B-B \# A$ :

$$
\begin{aligned}
h \partial_{s} Q_{s}= & {\left[P_{s}, \check{\psi}_{s}\right]=\left[P, \check{\psi}_{s}\right]+\left[Q_{s}, \check{\psi}_{s}\right] } \\
= & {\left[P, \check{\psi}_{s}\right]+\widetilde{\mathcal{O}}\left(h\langle\xi\rangle^{M-1} \epsilon \frac{h}{\epsilon\langle\xi\rangle}\right)+\widetilde{\mathcal{O}}\left(s^{2} \epsilon\langle\xi\rangle^{M-2} \epsilon \frac{h}{\epsilon\langle\xi\rangle}\right) } \\
& +\widetilde{\mathcal{O}}\left(s \sqrt{\epsilon} \epsilon \frac{h}{\epsilon\langle\xi\rangle}\right) \#\left(P_{\xi}^{\prime}, \frac{1}{\langle\xi\rangle} P_{x}^{\prime}\right)+\widetilde{\mathcal{O}}(s \sqrt{\epsilon}) \# \widetilde{\mathcal{O}}\left(\epsilon\langle\xi\rangle^{M-1}\right) \frac{h}{\sqrt{\epsilon}\langle\xi\rangle} \\
= & {\left[P, \check{\psi}_{s}\right]+\widetilde{\mathcal{O}}\left(h^{2}\langle\xi\rangle^{M-2}\right)+\widetilde{\mathcal{O}}\left(s^{2} \epsilon h\langle\xi\rangle^{M-3}\right) } \\
& +\widetilde{\mathcal{O}}\left(\frac{s \sqrt{\epsilon} h}{\langle\xi\rangle}\right) \#\left(P_{\xi}^{\prime}, \frac{1}{\langle\xi\rangle} P_{x}^{\prime}\right)+\widetilde{\mathcal{O}}\left(s \epsilon h\langle\xi\rangle^{M-2}\right) .
\end{aligned}
$$

On the other hand,

$$
\begin{aligned}
{\left[P, \check{\psi}_{s}\right] } & =\frac{h}{i}\left\{P, \check{\psi}_{s}\right\}+\widetilde{\mathcal{O}}\left(\langle\xi\rangle^{M} \epsilon \frac{h^{3}}{\langle\xi\rangle^{3} \epsilon^{3 / 2}}\right) \\
& =i h\left\{\widehat{\psi}_{s} \circ \kappa(s), P\right\}+h\left(P_{\xi}^{\prime}, \frac{P_{x}^{\prime}}{\langle\xi\rangle}\right) \cdot \widetilde{\mathcal{O}}\left(\frac{h}{\langle\xi\rangle \epsilon^{1 / 2}}\right)+\widetilde{\mathcal{O}}\left(\epsilon^{-\frac{1}{2}} h^{3}\langle\xi\rangle^{M-3}\right) \\
& =i h\left\{\widehat{\psi}_{s} \circ \kappa(s), P\right\}+h \widetilde{\mathcal{O}}\left(\frac{h}{\langle\xi\rangle \epsilon^{1 / 2}}\right) \#\left(P_{\xi}^{\prime}, \frac{P_{x}^{\prime}}{\langle\xi\rangle}\right)+\widetilde{\mathcal{O}}\left(\frac{h^{3}}{\epsilon}\langle\xi\rangle^{M-3}\right),
\end{aligned}
$$

so

$$
\begin{aligned}
h \partial_{s} Q_{s}= & i h\left\{\widehat{\psi}_{s} \circ \kappa(s), P\right\}+\widetilde{\mathcal{O}}\left(\frac{h^{2}}{\epsilon^{1 / 2}\langle\xi\rangle}+\frac{s \epsilon^{1 / 2} h}{\langle\xi\rangle}\right) \#\left(P_{\xi}^{\prime}, \frac{P_{x}^{\prime}}{\langle\xi\rangle}\right) \\
& +\widetilde{\mathcal{O}}\left(h^{2}\langle\xi\rangle^{M-2}+s \epsilon h\langle\xi\rangle^{M-2}\right) .
\end{aligned}
$$

Since $\kappa(s)$ is obtained from integrating $i H_{\widehat{\psi}_{s}}$, we obtain, using also that $Q_{0}=0$,

$$
\begin{aligned}
Q_{\delta}= & P \circ \kappa(\delta)-P+\widetilde{\mathcal{O}}\left(\frac{\delta^{2} \sqrt{\epsilon}}{\langle\xi\rangle}+\frac{\delta h \epsilon^{-1 / 2}}{\langle\xi\rangle}\right) \#\left(P_{\xi}^{\prime}, \frac{P_{x}^{\prime}}{\langle\xi\rangle}\right) \\
& +\widetilde{\mathcal{O}}\left(h \delta\langle\xi\rangle^{M-2}+\delta^{2} \epsilon\langle\xi\rangle^{M-2}\right)
\end{aligned}
$$

under the assumption (5.60). (Recall that here $M$ is the order of $P$.)

\section{The conjugated pseudodifferential operator}

Let $P$ be the Operator introduced in the beginning of Section 2 satisfying the assumptions (2.1)-(2.7). To start with, we assume Hypothesis 4.1 and define $\mathcal{C}, \psi_{\epsilon}$ as in (4.5), (4.6). Later, we shall also use the dynamical Hypothesis 4.3 (imply$\operatorname{ing}(4.24))$.

The function $\psi_{\epsilon}$ satisfies $\psi_{\epsilon}=\widetilde{\mathcal{O}}(\epsilon), \xi \cdot \partial_{\xi} \psi_{\epsilon}=\widetilde{\mathcal{O}}\left(\epsilon\langle\xi\rangle^{-2}\right)$ in the sense of (4.27), (4.28), but we also know that these bounds improve considerably away from $\mathcal{C}$.

Let $A=A_{\delta}$ be an elliptic Fourier integral operator of order 0,0 quantizing $\kappa(\delta)$ as in Section 5 , and let $B_{\delta}$ be a corresponding Operator quantizing $\kappa(\delta)^{-1}$. 
Then we can apply Proposition 5.3 to see that $B A=\mathrm{Op}_{h}(c)$, where $c=\widetilde{\mathcal{O}}(1)$ is elliptic. Now restrict the attention to the intermediate region, where $\psi_{\epsilon}=\epsilon \psi(x)$, $\psi(x) \in C^{\infty}$. Using that $\epsilon=A h$ with $A$ large but fixed, we find here that

$$
e^{-\delta \psi_{\epsilon} / h} \circ A=\mathrm{Op}_{h}(c)
$$

where $c=\mathcal{O}(1)$ in the standard symbol sense, $\partial_{x}^{\alpha} \partial_{\xi}^{\beta} c=\mathcal{O}\left(\langle\xi\rangle^{-|\beta|}\right)$ and $c$ is elliptic in this class. Let $\widetilde{c}=\mathcal{O}(1)$ be an elliptic symbol which is equal to 1 in the interior region and which changes from 1 to $c$ when going outwards in the intermediate region. We now replace $A$ by $A \circ \mathrm{Op}_{h}(\widetilde{c})^{-1}$. (Here we assume that $A_{0}=1$, to avoid a topological difficulty.) Then we have achieved that $A u=e^{\delta \psi_{\epsilon} / h} u$ for $u$ supported in the exterior region including a shell where $\psi_{\epsilon}=\epsilon \psi, \psi=\psi(x) \in C^{\infty}$ as above and the region further out where $\psi_{\epsilon}=\psi_{\epsilon}(x)=\widetilde{\mathcal{O}}(\epsilon)$.

Next, we consider $P^{\delta}$ defined as in Proposition 5.9 by $P A_{\delta}=A_{\delta} P^{\delta}$. Recall that $P=P_{2}+i P_{1}+P_{0}$ and write $P_{j} A_{\delta}=A_{\delta} P_{j}^{\delta}, P^{\delta}=P+Q^{\delta}, P_{j}^{\delta}=P_{j}+Q_{j}^{\delta}$.

For $P_{j}, j=0,2$, we apply (5.62) and get on the symbol level over any bounded set in $x$-space

$$
\begin{aligned}
Q_{j}^{\delta}= & p_{j} \circ \kappa(\delta)-p_{j}+\widetilde{\mathcal{O}}\left(\frac{\delta^{2} \sqrt{\epsilon}}{\langle\xi\rangle}+\frac{\delta h \epsilon^{-\frac{1}{2}}}{\langle\xi\rangle}\right) \#\left(\partial_{\xi} p_{j}, \frac{\partial_{x} p_{j}}{\langle\xi\rangle}\right) \\
& +\widetilde{\mathcal{O}}\left(h \delta\langle\xi\rangle^{j-2}+\delta^{2} \epsilon\langle\xi\rangle^{j-2}\right) \\
= & \widetilde{\mathcal{O}}(\delta \sqrt{\epsilon}) \#\left(\partial_{\xi} p_{j}, \frac{\partial_{x} p_{j}}{\langle\xi\rangle}\right)+\widetilde{\mathcal{O}}\left(\left(h \delta+\delta^{2} \epsilon\right)\langle\xi\rangle^{j-2}\right) .
\end{aligned}
$$

For $p_{1}$ we need the more precise information about $\psi_{\epsilon}$ given in (4.10), (4.12), (4.13), satisfied also by $\psi_{\epsilon}$, as well as the fact that $\psi_{\epsilon}(\rho)$ is independent of $\epsilon$ in $\left|\rho-\rho_{j}\right| \leq \sqrt{\epsilon}$ (actually in $\left|\rho-\rho_{j}\right| \leq \sqrt{\epsilon} / C$ for some $C>0$, but we can always dilate in $\epsilon$ ). From these estimates it follows that

$$
\left(\frac{\partial_{x} p_{1}}{\langle\xi\rangle}, \partial_{\xi} p_{1}\right) \otimes\left(\partial_{x} \psi_{\epsilon},\langle\xi\rangle \partial_{\xi} \psi_{\epsilon}\right)=\widetilde{\mathcal{O}}(\epsilon)
$$

over a neighborhood of the set where $\psi_{\epsilon}$ also depends on $\xi$. It also follows that

$$
p_{1}(\kappa(\delta)(x, \xi))-p_{1}(x, \xi)=\widetilde{\mathcal{O}}(\delta \epsilon) .
$$

From Proposition 5.9, we deduce that

$$
Q_{1}^{\delta}=p_{1}(\kappa(\delta)(x, \xi))-p_{1}(x, \xi)+\widetilde{\mathcal{O}}(h) .
$$

By construction of $\kappa(\delta)$, especially (4.47), we see that

$$
p_{1}(\kappa(\delta)(x, \xi))-p_{1}(x, \xi)=i \delta H_{\psi_{\epsilon}} p_{1}(x, \xi)+\widetilde{\mathcal{O}}\left(\epsilon \delta^{2}\right) .
$$

In fact, this is quite obvious in the intermediate and far out regions, and near a point $\rho_{j} \in \mathcal{C}$, we choose canonical coordinates so that $\rho_{j}=(0,0)$ and put $\rho=\sqrt{\epsilon} \widetilde{\rho}$. Then if $\psi_{\epsilon}(\rho)=\epsilon \widetilde{\psi}_{\epsilon}(\widetilde{\rho})$, we get $H_{\psi_{\epsilon}}=\widetilde{H}_{\widetilde{\psi}_{\epsilon}}$, where the tilde on the $H$ indicates that we take the Hamilton field with respect to the $\widetilde{\rho}$-variables. The manifold 
$\Lambda_{\delta}: \operatorname{Im} \rho=\delta H_{\psi_{\epsilon}}(\operatorname{Re} \rho)$ now becomes $\operatorname{Im} \widetilde{\rho}=\delta \widetilde{H}_{\widetilde{\psi}_{\epsilon}}(\operatorname{Re} \widetilde{\rho})$ while $p_{1}=\mathcal{O}\left(\epsilon \widetilde{\rho}^{2}\right)$ and since $\psi_{\epsilon}$ satisfies (4.10),

$$
\partial_{\widetilde{\rho}}^{\alpha} \widetilde{\psi}_{\epsilon}=\mathcal{O}\left(\frac{1}{\langle\widetilde{\rho}\rangle^{|\alpha|}}\right) .
$$

Then $\kappa(\delta)(\rho)$ becomes $\widetilde{\kappa}(\delta)(\widetilde{\rho})=\widetilde{\rho}+i \delta \widetilde{H}_{\widetilde{\psi}_{\epsilon}}(\widetilde{\rho})+\mathcal{O}\left(\delta^{2}\langle\widetilde{\rho}\rangle^{-2}\right)$, so $p_{1}(\widetilde{\kappa}(\delta) \widetilde{\rho})=p_{1}(\rho)+$ $i \delta \widetilde{H}_{\widetilde{\psi}_{\epsilon}}\left(p_{1}\right)+\mathcal{O}\left(\epsilon \delta^{2}\right)$, leading to $(6.5)$.

It follows that

$$
Q_{1}^{\delta}=-i \delta H_{p_{1}} \psi_{\epsilon}+\widetilde{\mathcal{O}}\left(\delta^{2} \epsilon+h\right)=\widetilde{\mathcal{O}}(\delta \epsilon+h) .
$$

Away from any neighborhood $\mathcal{C}$ we have the improved estimates

$$
Q_{1}^{\delta}=-i \delta H_{p_{1}} \psi_{\epsilon}+\mathcal{O}\left(\delta^{2} \epsilon+h\right)
$$

in the usual symbol sense, as long as we stay away from the outer region where $\psi_{\epsilon}=\psi_{\epsilon}(x)$ only satisfies $\psi_{\epsilon}=\widetilde{\mathcal{O}}(\epsilon)$, and where we can apply the analysis of Section 2 , so

$$
Q_{1}^{\delta}=-i \delta H_{p_{1}} \psi_{\epsilon}=-i \delta \nu\left(\psi_{\epsilon}\right)
$$

there.

\section{Estimates for the conjugated pseudodifferential operator and localization of the spectrum}

Let $P^{\delta}$ be the conjugated Operator of Section 6 . We shall study lower bounds for

$$
\operatorname{Re}\left(P^{\delta} u \mid u\right)=\operatorname{Re}\left(\left(P_{0}^{\delta}+P_{2}^{\delta}\right) u \mid u\right)-\operatorname{Im}\left(P_{1}^{\delta} u \mid u\right), \quad u \in \mathcal{S}(M) .
$$

Using (6.1), we get

$$
\begin{aligned}
\operatorname{Re}\left(P_{2}^{\delta} u \mid u\right) & \geq\left(P_{2} u \mid u\right)-C \delta \sqrt{\epsilon}\|u\|\left\|\operatorname{Op}\left(\left(\partial_{\xi} p_{2}, \frac{\partial_{x} p_{2}}{\langle\xi\rangle}\right)\right) u\right\|-C\left(h \delta+\delta^{2} \epsilon\right)\|u\|^{2} \\
& \geq\left(P_{2} u \mid u\right)-\frac{1}{C_{1}}\left\|\operatorname{Op}\left(\left(\partial_{\xi} p_{2}, \frac{\partial_{x} p_{2}}{\langle\xi\rangle}\right)\right) u\right\|^{2}-C_{2}\left(h \delta+\delta^{2} \epsilon\right)\|u\|^{2},
\end{aligned}
$$

where $C_{1}$ can be chosen arbitrarily large and $C_{2}$ depends on $C_{1}$. Here

$$
\frac{1}{2} p_{2}-\frac{1}{C_{1}}\left(\partial_{\xi} p_{2}, \frac{\partial_{x} p_{2}}{\langle\xi\rangle}\right)^{2} \geq 0,
$$

if $C_{1}$ is large enough, so

$$
\begin{aligned}
\left(P_{2} u \mid u\right)-\frac{1}{C_{1}}\left\|\operatorname{Op}\left(\left(\partial_{\xi} p_{2}, \frac{\partial_{x} p_{2}}{\langle\xi\rangle}\right)\right) u\right\|^{2}= & \left(\mathrm{Op}\left(p_{2}-\frac{1}{C_{1}}\left(\partial_{\xi} p_{2}, \frac{\partial_{x} p_{2}}{\langle\xi\rangle}\right)^{2}\right) u \mid u\right) \\
& +\mathcal{O}\left(h^{2}\right)\|u\|^{2} \\
\geq & \frac{1}{2}\left(P_{2} u \mid u\right)-\mathcal{O}\left(h^{2}\right)\|u\|^{2},
\end{aligned}
$$


where the estimate follows from the Fefferman-Phong inequality in the semiclassical setting (see [14]). It follows that

$$
\operatorname{Re}\left(P_{2}^{\delta} u \mid u\right) \geq \frac{1}{2}\left(P_{2} u \mid u\right)-C\left(h^{2}+h \delta+\delta^{2} \epsilon\right)\|u\|^{2} .
$$

For $P_{0}$ we have the same conclusion,

$$
\operatorname{Re}\left(P_{0}^{\delta} u \mid u\right) \geq \frac{1}{2}\left(P_{0} u \mid u\right)-C\left(h^{2}+h \delta+\delta^{2} \epsilon\right)\|u\|^{2},
$$

since the same analysis applies for $x$ in a bounded region and further out, we just have $P_{0}^{\delta}=P_{0} \geq 0$.

Combining this with (6.6), we get

$$
\operatorname{Re}\left(P^{\delta} u \mid u\right) \geq \delta\left(\mathrm{Op}\left(H_{p_{1}} \psi_{\epsilon}\right) u \mid u\right)+\frac{1}{2}\left(\left(P_{0}+P_{2}\right) u \mid u\right)-C\left(h+h \delta+\delta^{2} \epsilon\right)\|u\|^{2} .
$$

Here we recall that $H_{p_{1}} \psi_{\epsilon}=\left\langle\widetilde{p}_{\epsilon}\right\rangle_{T_{0}}-\widetilde{p}_{\epsilon}$ by (4.7), that $\left\langle\widetilde{p}_{\epsilon}\right\rangle_{T_{0}}$ satisfies (4.25), (4.26) and that $\widetilde{p}_{\epsilon} \leq \widetilde{p} \leq p_{0}+p_{2}$ by (4.16). Write,

$$
\begin{aligned}
\delta H_{p_{1}} \psi_{\epsilon}+\frac{1}{2}\left(p_{0}+p_{2}\right)= & \delta\left\langle\widetilde{p}_{\epsilon}\right\rangle_{T_{0}}-\delta \widetilde{p}_{\epsilon}+\frac{1}{2}\left(p_{0}+p_{2}\right) \\
= & \delta\left\langle\widetilde{p}_{\epsilon}\right\rangle_{T_{0}}+\delta\left(\widetilde{p}-\widetilde{p}_{\epsilon}\right)+\delta\left(p_{0}+p_{2}-\widetilde{p}\right) \\
& +\left(\frac{1}{2}-\delta\right)\left(p_{0}+p_{2}\right) .
\end{aligned}
$$

Here we want a lower bound for $\mathrm{Op}\left(\widetilde{p}-\widetilde{p}_{\epsilon}\right)$. This is quite straight forward away from $\mathcal{C}$, so we concentrate on a neighborhood of a point $\rho_{j} \in \mathcal{C}$. Assume $\rho_{j}=0$ for simplicity. Then near 0 we have by (4.15)

$$
\widetilde{p}_{\epsilon}=g\left(\frac{|\rho|^{2}}{\epsilon}\right) \widetilde{p}, \quad \widetilde{p}-\widetilde{p}_{\epsilon}=\left(1-g\left(\frac{|\rho|^{2}}{\epsilon}\right)\right) \widetilde{p}=\left(1-\ell_{\epsilon}(\rho)\right)^{2} \widetilde{p},
$$

where

$$
\ell_{\epsilon}(\rho)=\ell\left(\frac{|\rho|^{2}}{\epsilon}\right)=\widetilde{\mathcal{O}}\left(\frac{\epsilon}{\epsilon+|\rho|^{2}}\right),
$$

and we may assume that $g$ has been chosen so that $1-g=(1-\ell)^{2}$ with $\ell$ smooth. Here

$$
\begin{aligned}
\left(1-\ell_{\epsilon}\right) \# \widetilde{p} \#\left(1-\ell_{\epsilon}\right) & =\widetilde{p}-\ell_{\epsilon} \# \widetilde{p}-\widetilde{p} \# \ell_{\epsilon}+\ell_{\epsilon} \# \widetilde{p} \# \ell_{\epsilon} \\
& =\left(1-\ell_{\epsilon}(\rho)\right)^{2} \widetilde{p}+\widetilde{\mathcal{O}}(h) .
\end{aligned}
$$

From this we conclude that

$$
\left(\mathrm{Op}\left(\widetilde{p}-\widetilde{p}_{\epsilon}\right) u \mid u\right) \geq-C h\|u\|^{2} .
$$

Similarly,

$$
\left(\mathrm{Op}\left(p_{0}+p_{2}-\widetilde{p}\right) u \mid u\right) \geq-C h^{2}\|u\|^{2},
$$

by the Fefferman-Phong inequality or by a direct argument. 
Let $0 \leq k_{\epsilon}=\widetilde{\mathcal{O}}(\epsilon)$ be equal to $\epsilon$ in $\mathcal{C}+B(0, \sqrt{\epsilon})$ and have its support in $\mathcal{C}+$ $B(0, \sqrt{2 \epsilon})$. Let $K_{\epsilon}=\mathrm{Op}\left(k_{\epsilon}\right)$. By $(4.25),(4.26)$ we have $\left\langle\widetilde{p}_{\epsilon}\right\rangle_{T_{0}} \backsim \min \left(\epsilon, \operatorname{dist}(\cdot, \mathcal{C})^{2}\right)$, $\mathrm{SO}$

$$
\epsilon \backsim k_{\epsilon}+\left\langle\widetilde{p}_{\epsilon}\right\rangle_{T_{0}}=\widetilde{\mathcal{O}}(\epsilon) .
$$

Hence,

$$
\left(\left(K_{\epsilon}+\operatorname{Op}\left(\left\langle\widetilde{p}_{\epsilon}\right\rangle_{T_{0}}\right)\right) u \mid u\right) \backsim \epsilon\|u\|^{2} .
$$

Combining (7.4)-(7.9), we get

Proposition 7.1. We have

$$
\begin{aligned}
\operatorname{Re}\left(\left(P^{\delta}+K_{\epsilon}\right) u \mid u\right) \geq \frac{\delta \epsilon}{C}\|u\|^{2}+\left(\frac{1}{2}-\delta\right)\left(\left(P_{2}+P_{0}\right) u \mid u\right)-C h\|u\|^{2}, & \\
u & \in \mathcal{S}(M) .
\end{aligned}
$$

Here we recall that $\delta>0$ should be small enough, $\epsilon=A h$ with $A$ arbitrarily large and fixed, and $C$ in (7.10) is independent of $\delta, A$ while $h$ is small enough depending on these two parameters. From (7.10) we get the a priori estimate

$$
\left(\frac{\delta A h}{C}-C h-\operatorname{Re} z\right)\|u\| \leq\left\|\left(P^{\delta}+K_{\epsilon}-z\right) u\right\|, \quad u \in \mathcal{S}(M),
$$

when $\operatorname{Re} z<\delta A h / C-C h$.

From Section 3 we know that $P$ has no spectrum in the open left half-plane. We shall next prove

Proposition 7.2. For every constant $B>0$ there is a constant $D>0$ such that $P$ has no spectrum in

$$
\{z \in \mathbf{C} ; \operatorname{Re} z<B h,|\operatorname{Im} z|>D h\}
$$

when $h>0$ is small enough. Moreover $\left\|(P-z)^{-1}\right\|=\mathcal{O}_{B}\left(h^{-1}\right)$ for $z$ in the set (7.12).

Proof. Choose $\delta>0$ small, then $A$ large enough, so that $\frac{\delta A h}{C}-C h-\operatorname{Re} z \geq$ $\epsilon /$ Const. when Re $z<B h$. Then (7.11) gives

$$
\frac{\epsilon}{C_{0}}\|u\| \leq\left\|\left(P^{\delta}+K_{\epsilon}-z\right) u\right\|, \quad u \in \mathcal{S}(M) .
$$

Take $z$ in the set (7.12). When $\operatorname{Re} z<0$, we already know that $z \notin \sigma(P)$, so we may assume that $0 \leq \operatorname{Re} z<B h$.

Now recall that the symbol of $K_{\epsilon}$ is $\widetilde{\mathcal{O}}(\epsilon)$ and supported in $\mathcal{C}+B(0, \sqrt{2 \epsilon})$. On that set we have $p^{\delta}=\widetilde{\mathcal{O}}(\epsilon)=\widetilde{\mathcal{O}}(A h)$ and hence

$$
\left|p^{\delta}-z\right|>\frac{\epsilon+|z|}{C_{0}}, \quad \text { when } \quad|\operatorname{Im} z|>D h,
$$

and $D$ is large enough, assuming still that $0 \leq \operatorname{Re} z<B h$.

It follows that we can find $E=\widetilde{\mathcal{O}}(\epsilon /(\epsilon+|z|))$ such that

$$
K_{\epsilon}=E \circ\left(P^{\delta}-z\right)+F, \quad F=\widetilde{\mathcal{O}}(h),
$$


where $E, F$ also denote the corresponding $h$-pseudodifferential operators. In particular,

$$
\left\|K_{\epsilon} u\right\| \leq \mathcal{O}(1)\left\|\left(P^{\delta}-z\right) u\right\|+\mathcal{O}(h)\|u\|,
$$

where the $\mathcal{O}_{\text {s }}$ are uniform in $\epsilon$. Combining this with (7.13) with $\epsilon \gg h$, we get

$$
\epsilon\|u\| \leq \mathcal{O}(1)\left\|\left(P^{\delta}-z\right) u\right\|, \quad u \in \mathcal{S}(M),
$$

for $0 \leq \operatorname{Re} z<B h, 0 \leq \operatorname{Im} z<D h$. Now recall that $P^{\delta}=A_{\delta}^{-1} P A_{\delta}$, where $A_{\delta}, A_{\delta}^{-1}: \mathcal{S} \rightarrow \mathcal{S}, L^{2} \rightarrow L^{2}$ and have $L^{2}$ norm $\mathcal{O}_{A}(1)$. Then (7.15) gives

$$
h\|u\| \leq \mathcal{O}_{B}(1)\|(P-z) u\|, \quad u \in \mathcal{S}(M),
$$

for $z$ in the set (7.12). From Section 3 we then know that $z \notin \sigma(P)$ and that $\left\|(P-z)^{-1}\right\| \leq \mathcal{O}_{B}(1) / h$.

\section{Asymptotics of eigenvalues}

Let $\rho_{j} \in \mathcal{C}$ and let $F_{\rho_{j}}$ be the matrix of the linearization of $H_{p}$ at $\rho_{j}$ (the so called fundamental matrix of $p$ at the doubly characteristic point $\rho_{j}$ ). Thanks to the fact that the quadratic approximation of $p^{\delta}=p \circ \kappa(\delta)$ at $\rho_{j}$ is elliptic on $T_{\rho_{j}}\left(\Lambda_{\delta}\right)$ and takes its values in a closed angle contained in the union of $\{0\}$ and the open right half plane, we know from [22] that the eigenvalues of $F_{\rho_{j}}$ are of the form $\pm \lambda_{j, k}$, $1 \leq k \leq n$, when repeated with their multiplicity, with $\operatorname{I} m \lambda_{j, k}>0$. Let

$$
\widetilde{\operatorname{tr}}\left(p, \rho_{j}\right)=\frac{1}{i} \sum_{k} \lambda_{j, k} .
$$

In our case the subprincipal symbol of $P$ at $\rho_{j}$ is zero and will not enter into the description of the eigenvalues.

Put

$$
q(x, \xi)=-p(x, i \xi)=p_{2}+p_{1}-p_{0} .
$$

Let $F_{q}, F_{p}$ be the fundamental matrices of $q, p$ at one of the critical points $\rho_{j} \in \mathcal{C}$. Since

$$
H_{q}(x, \xi)=\frac{1}{i}\left(p_{\xi}^{\prime}(x, \eta) \cdot \frac{\partial}{\partial x}-p_{x}^{\prime}(x, \eta) \cdot \frac{\partial}{\partial \eta}\right), \quad \text { with } \quad \eta=i \xi,
$$

we see that $F_{q}$ and $\frac{1}{i} F_{p}$ have the same eigenvalues; $\pm \frac{1}{i} \lambda_{k}, k=1, \ldots, n$ ( $j$ being fixed) where $\operatorname{Re}\left(\frac{1}{i} \lambda_{k}\right)>0$. Now $q$ is real-valued and we can apply the stable manifold theorem as in [9] (and at many other places) to see that the $H_{q}$-flow has a stable outgoing manifold $\Lambda_{+}$passing through $\rho_{j}$ such that $T_{\rho_{j}} \Lambda_{+}^{\mathrm{C}}$ is spanned by the generalized eigenvectors corresponding to $+\frac{1}{i} \lambda_{k}, k=1, \ldots, n$. We also know that $\Lambda_{+}$is a Lagrangian manifold and that $q$ vanishes on $\Lambda_{+}$.

Lemma 8.1. Assume for simplicity that $\rho_{j}=(0,0)$. Then $T_{\rho_{j}} \Lambda_{+}$is transversal both to $\{x=0\}$ and to $\{\xi=0\}$. 
Proof. Since we consider the linearized situation we may assume right away that $q$ is a quadratic form (the second order Taylor polynomial at $\rho_{j}$ ), so that $p_{j}$ are quadratic forms as well. The dynamical condition (4.21) implies that

$$
\left\langle p_{0}+p_{2}\right\rangle_{T_{0}}>0 \quad \text { as a quadratic form . }
$$

Let $L=\Lambda_{+} \cap\{x=0\}$. Since $p_{0}=0, p_{1}=0$ on $\{x=0\}$, we know that $p_{2}=0$ on $L$ and hence $H_{q}=H_{p_{1}}$ on $L$. Now $H_{q}$ is tangent to $\Lambda_{+}$and $H_{p_{1}}$ is tangent to $\{x=0\}$, so $H_{q}=H_{p_{1}}$ is tangent to $L$. Thus $L$ is an $H_{q^{-}}$and $H_{p_{1}}$ invariant subspace on which $p_{2}+p_{0}=0$, and since $\left\langle p_{2}+p_{0}\right\rangle_{T_{0}}>0$ away from 0 , we necessarily have $L=0$.

The proof of the fact that $\Lambda_{+} \cap\{\xi=0\}=0$ is the same after permuting the roles of $p_{0}$ and $p_{2}$.

It follows from the lemma that

$$
\Lambda_{+}: \xi=\phi_{+}^{\prime}(x), \quad x \in \operatorname{neigh}(0),
$$

where $\phi_{+} \in C^{\infty}(\operatorname{neigh}(0) ; M), \phi_{+}(0)=0, \phi_{+}^{\prime}(0)=0$, $\operatorname{det} \phi_{+}^{\prime \prime}(0) \neq 0$.

Let $\Lambda_{-}$be the stable incoming $H_{q}$-invariant manifold such that $T_{\rho_{j}} \Lambda_{-}^{\mathbf{C}}$ is spanned by the generalized eigenvectors of $F_{q}$ corresponding to $-\frac{1}{i} \lambda_{k}, 1 \leq k \leq n$. The lemma is valid also for $\Lambda_{-}$and (8.3) has an obvious analogue for $\Lambda_{-}$where we let $\phi_{-}$denote the corresponding generating function.

Proposition 8.2. We have $\phi_{+}^{\prime \prime}(0)>0, \phi_{-}^{\prime \prime}(0)<0$.

Proof. Again we can consider the linearized quadratic case. If we make a smooth deformation of $q$, then $\Lambda_{+}, \Lambda_{-}, \phi_{+}^{\prime \prime}(0), \phi_{-}^{\prime \prime}(0)$ vary smoothly with the deformation parameter and $\operatorname{det} \phi_{ \pm}^{\prime \prime}(0) \neq 0$, provided of course that we maintain the condition (8.2). Consider the deformation from $q=q_{0}$ to to $\xi^{2}-x^{2}=q_{1}$ :

$$
q_{t}(x, \xi)=(1-t) q(x, \xi)+t\left(\xi^{2}-x^{2}\right)=p_{2}^{t}+p_{1}^{t}-p_{0}^{t},
$$

with

$$
p_{2}(\xi)=(1-t) p_{2}(\xi)+t \xi^{2}, \quad p_{0}^{t}(x)=(1-t) p_{0}(x)+t x^{2} .
$$

$p_{2}^{t}$ and $p_{0}^{t}$ are positive definite for $t>0$, so (8.2) is maintained. For $t=1$ we have $\phi_{ \pm}^{1}(x)= \pm x^{2} / 2$ so $\pm \phi_{ \pm}^{\prime \prime}(0)$ is positive definite. Since the signatures of $\phi_{ \pm}^{\prime \prime}(0)$ are independent of $t$, we get the lemma.

From Section 6 we recall that the conjugated operator $P_{\delta}=P_{\delta, \epsilon}$ has the symbol

$$
p+\delta H_{p_{1}} \psi_{\epsilon}+\sum_{j=0,2} \widetilde{\mathcal{O}}(\delta \sqrt{\epsilon}) \#\left(\partial_{\xi} p_{j}, \frac{\partial_{x} p_{j}}{\langle\xi\rangle}\right)+\widetilde{\mathcal{O}}\left(h+\delta^{2} \epsilon\right)
$$

and that we have the a priori estimate (7.10) expressing that the real part of $P^{\delta}$ is $\geq \frac{\delta \epsilon}{C}-C h$ outside $\mathcal{C}+B(0, \sqrt{\epsilon})$. In the set $\mathcal{C}+B(0, \sqrt{\epsilon})$ the symbol $P^{\delta}$ is independent of $\epsilon$ modulo $\mathcal{O}\left(\epsilon\left(\frac{h}{\epsilon}\right)^{\infty}\right)$ and is of the form $\widetilde{P}_{\delta} \sim p^{\delta}+h r_{1}+h^{2} r_{2}+\cdots$, where $\left|p^{\delta}\right| \backsim \operatorname{dist}(\cdot, \mathcal{C})^{2}, \operatorname{Re} p^{\delta} \backsim \operatorname{dist}(\cdot, \mathcal{C})^{2}$. In the following we shall assume for 
a while (in order to simplify the notations) that $\mathcal{C}$ is reduced to a single point $\rho_{0}=(0,0)$.

We fix $B>0$ and restrict the spectral parameter $z$ to the disc $D(0, B h)$. We shall take $\epsilon=A h$ with $A \gg B$ sufficiently large. Assume that $M=\mathbf{R}^{n}$ for simplicity. Following basically [14] we recall the construction of a well-posed Grushin problem, first for $\widetilde{P}_{\delta}-z$ and then for $P_{\delta}-z$. Let $\Lambda=\left(x^{2}+\left(h D_{x}\right)^{2}\right)^{1 / 2}$ so that $\widetilde{P}_{\delta}$ is equipped with the natural domain $\mathcal{D}\left(\widetilde{P}_{\delta}\right)=\left\{u \in L^{2} ; \Lambda^{2} u \in L^{2}\left(\mathbf{R}^{n}\right)\right\}$. In Section 11 of [14] the authors constructed operators

$$
R_{-}: \mathbf{C}^{N_{0}} \rightarrow L^{2}, \quad R_{+}: L^{2} \rightarrow \mathbf{C}^{N_{0}}
$$

of the form

$$
R_{-} u_{-}=\sum_{j=1}^{N_{0}} u_{-}(j) e_{j}^{h}(x), \quad R_{+} u(j)=\left(u \mid f_{j}^{h}(x)\right),
$$

with the following properties:

$$
\begin{aligned}
e_{j}^{h}(x) & =h^{-\frac{n}{4}} e_{j}\left(\frac{x}{\sqrt{h}}\right), & f_{j}^{h}(x) & =h^{-\frac{n}{4}} f_{j}\left(\frac{x}{\sqrt{h}}\right), \\
e_{j}(x) & =p_{j}(x) e^{i \Phi_{0}(x)}, & f_{j}(x) & =q_{j}(x) e^{i \Psi_{0}(x)},
\end{aligned}
$$

where $p_{j}, q_{j}$ are polynomials and $\Phi_{0}, \Psi_{0}$ are quadratic forms with $\operatorname{Im} \Phi_{0}, \operatorname{Im} \Psi_{0}>$ $0, \Phi_{0}^{\prime \prime}=\phi_{+}^{\prime \prime}(0)$. If $\delta>0$ is small and fixed, $A$ sufficiently large, the problem

$$
\left(\widetilde{P}_{\delta}-z\right) u+R_{-}^{\delta} u_{-}=v, \quad R_{+}^{\delta} u=v_{+},
$$

for $v \in L^{2}\left(\mathbf{R}^{n}\right), v_{+} \in \mathbf{C}^{N_{0}}$ has a unique solution $u \in \mathcal{D}\left(\widetilde{P}_{\delta}\right), u_{-} \in \mathbf{C}^{N_{0}}$, where $R_{+}^{\delta}=R_{+} A_{\delta}, R_{-}^{\delta}=A_{\delta}^{-1} R_{-}$, Moreover, for the solution, we have the a priori estimate

$$
\left\|\Lambda^{2} u\right\|+\left|u_{-}\right| \leq C\left(\|v\|+h\left|v_{+}\right|\right) .
$$

Notice here that $\left(h^{-1 / 2} \Lambda\right)^{N} R_{-}=\mathcal{O}(1): \mathbf{C}^{N_{0}} \rightarrow L^{2}$, for every $N \in \mathbf{R}$ and similarly for $R_{+}, R_{ \pm}^{\delta}$. From this, it follows that $R_{-}^{\delta}=R_{-}^{\delta, \epsilon}$ depends weakly on $\epsilon$ in the sense that if $\widetilde{\epsilon}=\widetilde{A} h, \widetilde{A} \geq A$, then

$$
\left\|R_{-}^{\delta, \widetilde{\epsilon}}-R_{-}^{\delta, \epsilon}\right\|_{\mathcal{L}\left(\mathbf{C}^{\left.N_{0}, L^{2}\right)}\right.}=\mathcal{O}\left(\frac{1}{A}\right)
$$

and similarly for $R_{+}^{\delta}$.

We shall derive an a priori estimate for the problem

$$
\left(P_{\delta}-z\right) u+R_{-}^{\delta} u_{-}=v, \quad R_{+}^{\delta} u=v_{+},
$$

when $u \in \mathcal{S}$. Let $\chi \in C_{0}^{\infty}(B(0,2))$ be equal to one on $B(0,1)$, and put $\chi_{\sqrt{\epsilon}}(x, \xi)=$ $\chi\left(\epsilon^{-1 / 2}(x, \xi)\right)$. We use the same notation for the corresponding $h$-quantization. We may assume that $\widetilde{P}_{\delta}-P_{\delta}=\widetilde{\mathcal{O}}\left(\epsilon(h / \epsilon)^{\infty}\right)$ on $\operatorname{supp}\left(\chi_{\sqrt{\epsilon}}\right)$. From the first equation 
in (8.10), we get

$$
\left\{\begin{array}{c}
\left(\widetilde{P}_{\delta}-z\right) \chi_{\sqrt{\epsilon}} u+R_{-}^{\delta} u_{-}=\chi_{\sqrt{\epsilon}} v+\chi_{\sqrt{\epsilon}}\left(\widetilde{P}_{\delta}-P_{\delta}\right) u \\
\quad+\left[\widetilde{P}_{\delta}, \chi_{\sqrt{\epsilon}}\right] u+\left(1-\chi_{\sqrt{\epsilon}}\right) R_{-} u_{-}, \\
R_{+}^{\delta} \chi_{\sqrt{\epsilon}} u=v_{+}-R_{+}^{\delta}\left(1-\chi_{\sqrt{\epsilon}}\right) u .
\end{array}\right.
$$

Here

$$
\begin{aligned}
\chi_{\sqrt{\epsilon}} \#\left(\widetilde{P}_{\delta}-P_{\delta}\right) & =\widetilde{\mathcal{O}}\left(h \frac{h}{\epsilon}\right) \\
{\left[\widetilde{P}_{\delta}, \chi_{\sqrt{\epsilon}}\right] } & =\widetilde{\mathcal{O}}\left(h \frac{h}{\epsilon}\right) \\
\left\|\left(1-\chi_{\sqrt{\epsilon}}\right) R_{-}^{\delta} u_{-}\right\| & =\left\|\left(1-\chi_{\sqrt{\epsilon}}\right)\left(h^{-\frac{1}{2}} \Lambda\right)^{-N}\left(h^{-\frac{1}{2}} \Lambda\right)^{N} R_{-}^{\delta} u_{-}\right\| \\
& =\mathcal{O}\left(\left(\frac{h}{\epsilon}\right)^{\frac{N}{2}}\right)\left|u_{-}\right|, \\
h\left|R_{+}^{\delta}\left(1-\chi_{\sqrt{\epsilon}}\right) u\right| & =h\left|R_{+}^{\delta}\left(h^{-\frac{1}{2}} \Lambda\right)^{N}\left(h^{-\frac{1}{2}} \Lambda\right)^{-N}\left(1-\chi_{\sqrt{\epsilon}}\right) u\right| \\
& =\mathcal{O}\left(\left(\frac{h}{\epsilon}\right)^{\frac{N}{2}} h\|u\|\right) .
\end{aligned}
$$

Thus, applying the a priori estimate (8.9) to (8.11), we get

$$
\left\|\Lambda^{2} \chi_{\sqrt{\epsilon}} u\right\|+\left|u_{-}\right| \leq C\left(\left\|\chi_{\sqrt{\epsilon}} v\right\|+\mathcal{O}\left(h \frac{h}{\epsilon}\right)\|u\|+\mathcal{O}\left(\frac{h}{\epsilon}\right)\left|u_{-}\right|+h\left|v_{+}\right|\right) .
$$

We next look for an a priori estimate for $\left(1-\chi_{\sqrt{\epsilon}}\right) u$. Apply $1-\chi_{\sqrt{\epsilon}}$ to $(8.10)$ :

$$
\left(P_{\delta}-z\right)\left(1-\chi_{\sqrt{\epsilon}}\right) u=\left(1-\chi_{\sqrt{\epsilon}}\right) v+\left[P_{\delta}, \chi_{\sqrt{\epsilon}}\right] u-\left(1-\chi_{\sqrt{\epsilon}}\right) R_{-} u_{-} .
$$

As before,

$$
\left[P_{\delta}, \chi_{\sqrt{\epsilon}}\right]=\left[\widetilde{P}_{\delta}, \chi_{\sqrt{\epsilon}}\right]+\left[P_{\delta}-\widetilde{P}_{\delta}, \chi_{\sqrt{\epsilon}}\right]=\mathcal{O}\left(h \frac{h}{\epsilon}\right): L^{2} \rightarrow L^{2},
$$

and using also (8.12), we get from (2.12), (8.15) that

$$
\begin{aligned}
\frac{\epsilon}{C}\left\|\left(1-\chi_{\sqrt{\epsilon}}\right) u\right\| \leq & \left\|\left(1-\chi_{\sqrt{\epsilon}}\right) v\right\|+\left\|K_{\epsilon}\left(1-\chi_{\sqrt{\epsilon}}\right) u\right\| \\
& +\mathcal{O}\left(\frac{h}{\epsilon} h\right)\|u\|+\mathcal{O}\left(\frac{h}{\epsilon}\right)\left|u_{-}\right|,
\end{aligned}
$$

where we can take $K_{\epsilon}(x, \xi)=\epsilon \chi\left(\frac{3(x, \xi)}{\sqrt{\epsilon}}\right)$. Since

$$
K_{\epsilon} \#\left(1-\chi_{\sqrt{\epsilon}}\right)=\mathcal{O}\left(\epsilon\left(\frac{h}{\epsilon}\right)^{N}\right): L^{2} \rightarrow L^{2},
$$

we get

$$
\frac{\epsilon}{C}\left\|\left(1-\chi_{\sqrt{\epsilon}}\right) u\right\| \leq\left\|\left(1-\chi_{\sqrt{\epsilon}}\right) v\right\|+\mathcal{O}\left(h \frac{h}{\epsilon}\right)\|u\|+\mathcal{O}\left(\frac{h}{\epsilon}\right)\left|u_{-}\right| .
$$


Let $L_{\epsilon}=\widetilde{\mathcal{O}}\left(\min \left(h+\operatorname{dist}(x, \xi ; 0,0)^{2}, \epsilon\right)\right)$ be an elliptic symbol in the class defined by the right hand side, so that

$$
\left\|\Lambda^{2} \chi_{\sqrt{\epsilon}} u\right\|+\epsilon\left\|\left(1-\chi_{\sqrt{\epsilon}}\right) u\right\| \backsim\left\|L_{\epsilon} u\right\| .
$$

Then summing (8.14), (8.17), we can absorb the various remainder terms to the right, and obtain

$$
\frac{1}{C}\left(\left\|L_{\epsilon} u\right\|+\left|u_{-}\right|\right) \leq\|v\|+h\left|v_{+}\right|,
$$

when (8.10) holds.

Now recall that $P_{\delta}, R_{+}^{\delta}, R_{-}^{\delta}$ have been defined from $P, R_{+}, R_{-}$by conjugation with $A_{\delta}$, and use also that

$$
\frac{1}{C} h\|u\| \leq\left\|L_{\epsilon} u\right\| \leq C A h\|u\|,
$$

to see that if $u, v \in \mathcal{S}(M)$ and

$$
\left\{\begin{array}{l}
(P-z) u+R_{-} u_{-}=v \\
R_{+} u=v_{+}
\end{array}, \quad z \in D(0, B h),\right.
$$

then

$$
h\|u\|+\left|u_{-}\right| \leq C\left(\|v\|+h\left|v_{+}\right|\right) .
$$

From the discussion after the proof of Corollary 3.2, we conclude that

$$
\mathcal{P}(z)=\left(\begin{array}{cc}
P-z & R_{-} \\
R_{+} & 0
\end{array}\right): \mathcal{D}(P) \times \mathbf{C}^{N_{0}} \rightarrow L^{2} \times \mathbf{C}^{N_{0}}
$$

is bijective with a bounded inverse

$$
\mathcal{E}=\left(\begin{array}{cc}
E(z) & E_{+}(z) \\
E_{-}(z) & E_{-+}(z)
\end{array}\right): L^{2} \times \mathbf{C}^{N_{0}} \rightarrow \mathcal{D}(P) \times \mathbf{C}^{N_{0}},
$$

for $z \in D(0, B h)$, and (8.20) shows that

$$
\begin{aligned}
\|E(z)\| & =\mathcal{O}\left(\frac{1}{h}\right), & \left\|E_{-}(z)\right\| & =\mathcal{O}(1), \\
\left\|E_{+}(z)\right\| & =\mathcal{O}(1), & \left\|E_{-+}(z)\right\| & =\mathcal{O}(h) .
\end{aligned}
$$

In Section 11 of [14] the authors studied the action of $\mathcal{P}(z)$ on spaces of functions of the form $\left(a(x ; h) e^{i \Phi_{0}(x) / h}, u_{-}\right)$, where $a$ is a symbol, and deduced that $E_{-+}(z ; h)$ has an asymptotic expansion in half powers of $h$ with a certain additional structure. From that was obtained the asymptotic expansion of the zeros of $\operatorname{det} E_{-+}$, i.e., of the eigenvalues of $P$ in $D(0, B h)$. That discussion goes through without any changes in the present situation, so we get the asymptotics for the eigenvalues in any disc $D(0, B h)$, when $h \rightarrow 0$. 
Theorem 8.3. We make the assumptions (2.1)-(2.7), (4.4), (4.21), (4.22), and recall the definition of $\mathcal{C}$ in $(4.5)$. Let $B>0$. Then there exists $h_{0}>0$ such that for $0<h \leq h_{0}$, the spectrum of $P$ in $D(0, B h)$ is discrete and the eigenvalues are of the form

$$
\lambda_{j, k}(h) \sim h\left(\mu_{j, k}+h^{1 / N_{j, k}} \mu_{j, k, 1}+h^{2 / N_{j, k}} \mu_{j, k, 2}+\cdots\right),
$$

where the $\mu_{j, k}$ are all the numbers in $D(0, B)$ of the form

$$
\mu_{j, k}=\frac{1}{i} \sum_{\ell=1}^{n} \nu_{j, k, \ell} \lambda_{j, \ell}+\frac{1}{2} \widetilde{\operatorname{tr}}\left(p, \rho_{j}\right), \quad \text { with } \quad \nu_{j, k, \ell} \in \mathbf{N},
$$

for some $j \in\{1, \ldots, N\}, N=\# \mathcal{C}$. (Possibly after changing $B$, we may assume that $\left.\left|\mu_{j, k}\right| \neq B, \forall j, k.\right)$ Recall here that $\pm \lambda_{j}$ are the eigenvalues of $F_{p}$. This description also takes into account the multiplicities in the natural way. If the coefficients $\nu_{j, k, \ell}$ in (8.25) are unique, then $N_{j, k}=1$ and we have only integer powers of $h$ in the asymptotic expansion (8.24).

Theorem 8.4. We make the same assumptions as in Theorem 8.3. For every B, $C>0$ there is a constant $D>0$ such that

$$
\left\|(z-P)^{-1}\right\| \leq \frac{D}{h}, \quad \text { for } \quad z \in D(0, B h) \quad \text { with } \quad \operatorname{dist}(z, \sigma(P)) \geq \frac{h}{C} .
$$

The last result follows from the formula

$$
(z-P)^{-1}=-E(z)+E_{+}(z) E_{-+}(z)^{-1} E_{-}(z),
$$

(8.23) and the fact that $\left\|E_{-+}^{-1}(z)\right\|=\mathcal{O}\left(h^{-1}\right)$ when (8.26) holds.

Still with $j=j_{0}$ fixed, let

$$
\mu=\frac{1}{i} \sum_{\ell=1}^{n} \nu_{\ell} \lambda_{\ell}+\frac{1}{2} \widetilde{\operatorname{tr}}\left(p, j_{0}\right), \quad \nu_{\ell} \in \mathbf{N}
$$

be a value as in (8.25) and assume that $\mu$ is simple in the sense that $\left(\nu_{1}, \ldots, \nu_{n}\right) \in$ $\mathbf{N}^{n}$ is uniquely determined by $\mu$. In particular, every $\lambda_{\ell}$ for which $\nu_{\ell} \neq 0$ is a simple eigenvalue of $F_{p}$. Then as in [9] (see also Chapter 3 in [21]) we can construct

$$
\lambda(h) \sim h\left(\mu+h \mu_{1}+h^{2} \mu_{2}+\cdots\right)
$$

with uniquely determined coefficients $\mu_{1}, \mu_{2}, \ldots$ and

$$
a(x ; h) \sim a_{0}(x)+h a_{1}(x)+\cdots \quad \text { in } C^{\infty}\left(\operatorname{neigh}\left(x_{j_{0}}\right)\right),
$$

where $a_{j}(x)=\mathcal{O}\left(\left|x-x_{j_{0}}\right|^{(m-2 j)_{+}}\right), m=\sum \nu_{\ell}$ and $a_{0}$ has a non-vanishing Taylor polynomial of order $m$, such that

$$
(P-\lambda(h))\left(a(x ; h) e^{-\phi_{+}(x) / h}\right)=\mathcal{O}\left(h^{\infty}\right) e^{-\phi_{+}(x) / h}
$$

in a neighborhood of $x_{j_{0}}$. Actually any neighborhood $\Omega \subset \subset \mathbf{R}^{n}$ will do, provided that

1) $\phi_{+}$is well-defined in a neighborhood of $\bar{\Omega}$.

2) $H_{\left.\right|_{\Lambda_{+}}} \neq 0$ on $\bar{\Omega} \backslash\left\{x_{j_{0}}\right\}$. 
3) $\Omega$ is star-shaped with respect to the point $x_{j_{0}}$ and the integral curves of the vector field $\nu_{+}:=\left(\pi_{x}\right)_{*}\left(H_{q_{\left.\right|_{\Lambda_{+}}}}\right)$, where $\pi_{x}((x, \xi))=x$.

We also know that $\lambda(h)$ is equal $\bmod \mathcal{O}\left(h^{\infty}\right)$ to the corresponding value in (8.24).

As in [9] we notice that if $\gamma \subset D(0, B)$ is a closed $h$-independent contour avoiding all the values $\mu_{j, k}$ in (8.25), and

$$
\pi_{h \gamma}=\frac{1}{2 \pi i} \int_{h \gamma}(z-P)^{-1} d z
$$

the corresponding spectral projection, then, using also Theorem 8.4,

$$
\left\|\pi_{h \gamma}\left(\chi a e^{-\phi_{+} / h}\right)-\chi a e^{-\phi_{+} / h}\right\|_{L^{2}}=\mathcal{O}\left(h^{\infty}\right)
$$

if $\chi \in C_{0}^{\infty}(\Omega)$ is equal to one near $x_{j_{0}}$. It follows that $\chi a e^{-\phi_{+} / h}$ is a linear combination of generalized eigenfunctions of $P$ with eigenvalues inside $h \gamma$ up to an error $\mathcal{O}\left(h^{\infty}\right)$ in $L^{2}$-norm.

\section{Exponentially weighted estimates}

In this section we keep the general assumptions on $P$ and assume for simplicity that $\mathcal{C}$ is reduced to a single point:

$$
\mathcal{C}=\{(0,0)\} .
$$

If $\psi \in C^{\infty}(\operatorname{neigh}(0, M) ; \mathbf{R})$, we have

$$
e^{\psi / h} \circ P \circ e^{-\psi / h}=P_{\psi},
$$

with the symbol (cf. (2.13))

$$
p_{\psi}(x, \xi)=p_{2}(x, \xi)-q\left(x, \psi^{\prime}(x)\right)+i\left(\partial_{\xi} q\right)\left(x, \psi^{\prime}(x)\right) \cdot \xi,
$$

where we recall that

$$
q(x, \xi)=p_{2}(x, \xi)+p_{1}(x, \xi)-p_{0}(x) .
$$

Notice that $\xi \mapsto q(x, \xi)$ is a convex function for every $x$.

Let $\phi=\phi_{+}(x) \in C^{\infty}(\operatorname{neigh}(0 ; \mathbf{R}))$ be the function introduced in Section 8 so that $\Lambda_{+}=\Lambda_{\phi}$ is the stable outgoing manifold through $(0,0)$ for the $H_{q}$-flow. Recall that by Proposition 8.2

$$
\phi^{\prime \prime}(0)>0 .
$$

We have the eikonal equation

$$
q\left(x, \phi^{\prime}(x)\right)=0,
$$

so $p_{\phi}(x, \xi)=p_{2}(x, \xi)+i\left(\partial_{\xi} q\right)\left(x, \phi^{\prime}(x)\right) \cdot \xi$. The vector field $\left(\partial_{\xi} q\right)\left(x, \phi^{\prime}(x)\right) \cdot \partial_{x}$ is the $x$-space projection of $H_{q_{\Lambda_{\phi}}}$, so its linearization at $x=0$ has all its eigenvalues with real part $>0$. Consequently (as we shall see in more detail in the proof of Lemma 10.1 below), there exists $G \in C^{\infty}$ (neigh $\left.(0, M) ; \mathbf{R}\right)$ such that

$$
\left(\partial_{\xi} q\right)\left(x, \phi^{\prime}(x)\right) \cdot \partial_{x} G \backsim x^{2}, \quad G(x) \backsim x^{2} .
$$


Let $\Omega_{G}(r)=\{x \in \operatorname{neigh}(0) ; G(x) \leq r\}$ for $0<r \ll 1$. Outside the set $\Omega_{G}\left(C_{0} \epsilon\right)$, we put

$$
\widehat{\psi}=\phi-\epsilon g(G),
$$

for a suitable function $g$ and for $0<\epsilon \ll 1$. (Eventually $\epsilon$ will be proportional to $h$.) Using (9.6), we get

$$
q\left(x, \widehat{\psi}^{\prime}(x)\right)=-\epsilon g^{\prime}(G)\left(\partial_{\xi} q\right)\left(x, \phi^{\prime}(x)\right) \cdot \partial_{x} G(x)+\epsilon^{2} g^{\prime}(G)^{2} p_{2}\left(x, G^{\prime}(x)\right) .
$$

Choose $g(G)=\ln G$ for $G \geq C_{0} \epsilon$, so that $g^{\prime}(G)=1 / G$. Then

$$
q\left(x, \widehat{\psi}^{\prime}(x)\right)=-\epsilon \frac{1}{G(x)}\left(\partial_{\xi} q\right)\left(x, \phi^{\prime}(x)\right) \cdot \partial_{x} G+\epsilon \frac{\epsilon}{G(x)} \frac{p_{2}\left(x, G^{\prime}(x)\right)}{G(x)} .
$$

Here

$$
\frac{1}{G}\left(\partial_{\xi} q\right)\left(x, \phi^{\prime}\right) \cdot \partial_{x} G \backsim 1, \quad \frac{p_{2}\left(x, G^{\prime}\right)}{G}=\mathcal{O}(1) .
$$

We conclude that

$$
q\left(x, \widehat{\psi}^{\prime}(x)\right) \leq-\frac{\epsilon}{C_{0}}, \quad x \in \operatorname{neigh}(0, M) \backslash \Omega_{G}\left(C_{0} \epsilon\right),
$$

if $C_{0}>0$ is large enough.

Outside a small fixed neighborhood of 0 we want to flatten out the weight. Let $f_{\delta}(t)=\delta f\left(\frac{t}{\delta}\right)$ be the function introduced in Section 2. For some small and fixed $\delta_{0}>0$, we put

$$
\psi=f_{\delta_{0}}(\widehat{\psi})=f_{\delta_{0}}(\phi-\epsilon g(G))
$$

which is also well-defined as the constant $3 \delta_{0} / 2$ for large $x$. From (9.11), the fact that $q(x, 0) \leq 0$ and the convexity of $q$, we get

$$
q\left(x, \psi^{\prime}(x)\right) \leq-\frac{\epsilon}{C} f_{\delta_{0}}^{\prime}(\widehat{\psi}),
$$

where we keep in mind that $0 \leq f_{\delta_{0}}^{\prime} \leq 1$.

We extend the definition of $\psi$ to a full neighborhood of $x=0$, by putting

$$
g(G)=\ln \left(C_{0} \epsilon\right)+\frac{1}{C_{0} \epsilon}\left(G-C_{0} \epsilon\right), \quad \text { for } \quad 0 \leq G \leq C_{0} \epsilon .
$$

Then in $\Omega_{G}\left(C_{0} \epsilon\right)$, we have $\widehat{\psi}^{\prime}=\psi^{\prime}=\phi^{\prime}-G^{\prime} / C_{0}$, so $q\left(x, \psi^{\prime}\right)=\mathcal{O}(\epsilon)$ by $(9.9)$.

In the exterior region where $\psi^{\prime}=f_{\delta_{0}}^{\prime}(\widehat{\psi}) \widehat{\psi}^{\prime}$ is small, the conjugated Operator $\widetilde{P}=P_{\psi}$ is close to the unperturbed Operator $P$ and we can apply the method of Section 2. Write

$$
\widetilde{p}=p_{2}+i \widetilde{p}_{1}+\widetilde{p}_{0}
$$

for the symbol of $\widetilde{P}$, so that by (9.3), we have

$$
\widetilde{p}_{1}=\left(\partial_{\xi} q\right)\left(x, \psi^{\prime}(x)\right) \cdot \xi, \quad \widetilde{p}_{0}=-q\left(x, \psi^{\prime}\right),
$$


to be compared with $p_{1}=\left(\partial_{\xi} q(x, 0)\right) \cdot \xi, p_{0}=-q(x, 0)$. Let $\widetilde{\nu}\left(x, \partial_{x}\right)=\sum \widetilde{c}_{j}(x) \partial_{x_{j}}$, where $\widetilde{p}_{1}=\sum \widetilde{c}_{j}(x) \xi_{j}$ and define $\widetilde{\psi}_{\epsilon}$ as in $(2.15)$, now for $\widetilde{P}$ instead of $P$ and with an additional cut-off:

$$
\widetilde{\psi}_{\epsilon}=(1-\chi(x)) \int k\left(\frac{t}{T_{0}}\right) f_{\epsilon} \circ \widetilde{p}_{0} \circ \exp (t \widetilde{\nu}) d t .
$$

Here $\chi \in C_{0}^{\infty}(M)$ is equal to 1 near $x=0$ and has its support in a small neighborhood of that point.

Then for $\widetilde{P}_{\delta \widetilde{\psi}_{\epsilon}}=e^{\delta \widetilde{\psi}_{\epsilon} / h} \circ \widetilde{P} \circ e^{-\delta \widetilde{\psi}_{\epsilon} / h}$ we get (cf. (2.22)),

$$
\widetilde{p}_{\delta \widetilde{\psi}_{\epsilon}} \geq(1-\delta) \widetilde{p}_{0}+\delta\left\langle f_{\epsilon} \circ \widetilde{p}_{0}\right\rangle_{T_{0}}-\mathcal{O}\left(\delta^{2} \epsilon\right) \text { outside supp } \chi
$$

where the time average in the second term to the right is taken along the trajectories of $\widetilde{\nu}$. In the region where $\chi=1$, we get $\widetilde{P}_{\delta \widetilde{\psi}_{c}}=\widetilde{P}$ of course, and in the intermediate region, $\operatorname{supp}(\nabla \chi)$, we have

$$
\widetilde{p}_{0, \delta \widetilde{\psi}_{\epsilon}}=\widetilde{p}_{0}+\mathcal{O}(\delta \epsilon) .
$$

If $\delta_{1}>0$ is small enough, we know that

$$
f_{\delta_{0}}^{\prime}(\widehat{\psi}(x)) \leq \delta_{1} \Rightarrow\left\langle f_{\epsilon} \circ \widetilde{p}_{0}\right\rangle_{T_{0}}(x) \geq \frac{\epsilon}{C_{1}},
$$

for some constant $C_{1}>0$. In fact, the $\nu$ and $\widetilde{\nu}$ trajectories through a given point with $f_{\delta_{0}}^{\prime}(\widehat{\psi}(x)) \leq \delta$ stay close for some fixed time $>0$, so the conditions (4.24), (4.23) imply that the $\widetilde{\nu}$-trajectory will encounter points with $p_{0} \geq 1 /$ Const during a non-trivial interval of time.

Then

- In the region where $f_{\delta_{0}}^{\prime}(\widehat{\psi}(x)) \leq \delta_{1}$, we have $\widetilde{p}_{0, \delta \widetilde{\psi}_{\epsilon}} \geq(1-\delta) \widetilde{p}_{0}+\frac{\delta \epsilon}{C_{1}}$ (and we recall that $\widetilde{p}_{0} \geq 0$ ).

- In the region where $f_{\delta_{0}}^{\prime}(\widehat{\psi}(x))>\delta_{1}$, and $G(x) \geq C_{0} \epsilon$, we have $\widetilde{p}_{0, \delta \widetilde{\psi}_{\epsilon}}=$ $\tilde{p}_{0}+\mathcal{O}(\delta \epsilon) \geq \frac{\epsilon \delta_{1}}{C}+\mathcal{O}(\delta \epsilon)$, by $(9.13)$.

- In $\Omega_{G}\left(C_{0} \epsilon\right)$, we have $\widetilde{p}_{0, \delta \widetilde{\psi}_{\epsilon}}=\widetilde{p}_{0}=\mathcal{O}(\epsilon)$.

Choosing first $\delta_{1}>0$ small enough, then $\delta>0$ small enough, we conclude that

$$
\widetilde{p}_{0, \delta \tilde{\psi}_{\epsilon}}\left\{\begin{array}{l}
\geq \frac{\epsilon}{C} \text { outside } \Omega_{G}\left(C_{0} \epsilon\right), \\
=\mathcal{O}(\epsilon) \text { in } \Omega_{G}\left(C_{0} \epsilon\right) .
\end{array}\right.
$$

Now $\widetilde{P}_{\delta \tilde{\psi}_{\epsilon}}=e^{\psi_{\epsilon} / h} \circ P \circ e^{-\psi_{\epsilon} / h}=P_{\psi_{\epsilon}}$, where

$$
\psi_{\epsilon}=\psi+\delta \widetilde{\psi}_{\epsilon},
$$

and where we recall that $\psi$ also depends on $\epsilon$. Combining Lemma 2.1 for $P_{\psi_{\epsilon}}$ with (9.20), we get

$$
\begin{aligned}
\int_{M \backslash \Omega_{G}\left(C_{0} \epsilon\right)}\left(\frac{\epsilon}{C}-\right. & \operatorname{Re} z)|u|^{2} d x+\int_{M}\left(P_{2} u\right) \bar{u} d x \\
& \leq \operatorname{Re}\left(\left(P_{\psi_{\epsilon}}-z\right) u \mid u\right)+\int_{\Omega_{G}\left(C_{0} \epsilon\right)}(\mathcal{O}(\epsilon)+\operatorname{Re} z)|u|^{2} d x .
\end{aligned}
$$


If Re $z=\mathcal{O}(h)$, we choose $\epsilon=A h$ with $A$ so large that $\frac{\epsilon}{C}-\operatorname{Re} z \geq h$ and deduce that

leading to the a priori estimate

$$
h\|u\|^{2}+\left(P_{2} u \mid u\right) \leq \operatorname{Re}\left(\left(P_{\psi_{\epsilon}}-z\right) u \mid u\right)+\mathcal{O}(h)\|u\|_{\Omega_{G}\left(C_{0} \epsilon\right)}^{2},
$$

$$
h\|u\| \leq\left\|\left(P_{\psi_{\epsilon}}-z\right) u\right\|+\mathcal{O}(h)\|u\|_{\Omega_{G}\left(C_{0} \epsilon\right)} .
$$

Re-injecting this estimate in (9.23), we get

$$
h^{2}\|u\|^{2}+h\left\|B^{\frac{1}{2}} h D u\right\|^{2} \leq(1+\alpha)\left\|\left(P_{\psi_{\epsilon}}-z\right) u\right\|^{2}+\mathcal{O}_{\alpha}\left(h^{2}\right)\|u\|_{\Omega_{G}\left(C_{0} \epsilon\right)}^{2},
$$

for every fixed $\alpha>0$.

Here $\left(P_{\psi_{\epsilon}}-z\right) u=e^{\psi_{\epsilon} / h}(P-z) v, u=e^{\psi_{\epsilon} / h} v$, so from (9.25) we get the a priori estimate for the original Operator

$$
\begin{aligned}
h\left\|e^{\psi_{\epsilon} / h} v\right\|+h^{\frac{1}{2}}\left\|B^{\frac{1}{2}} h D\left(e^{\psi_{\epsilon} / h} v\right)\right\| \leq & \mathcal{O}(1)\left\|e^{\psi_{\epsilon} / h}(P-z) v\right\| \\
& +\mathcal{O}(h)\left\|e^{\psi_{\epsilon} / h} v\right\|_{\Omega_{G}\left(C_{0} \epsilon\right)},
\end{aligned}
$$

uniformly, for $|\operatorname{Re} z| \leq C h$ provided that $\epsilon=A h$ for $A$ large enough depending on $C$.

Now let $\lambda(h)=\lambda_{1, k}(h)$ be an eigenvalue of $P$ as in (8.24), (8.28) and assume that $\mu$ is given by (8.27) and is simple, as explained after that equation. Then $\lambda(h)$ is a simple eigenvalue of $P$ and is the only eigenvalue in some disc $D\left(\lambda(h), h / C_{0}\right)$. Let $u_{\mathrm{WKB}}(x ; h)$ be the approximate solution given in (8.29), (8.30) and let $u=$ $\pi_{h \gamma}\left(\chi u_{\mathrm{BKW}}\right)$ be the corresponding exact eigenfunction, where $\gamma=\partial D\left(\mu, \frac{1}{2 C_{0}}\right)$.

Theorem 9.1. a) Outside any $h$-independent neighborhood of 0 , we have

$$
u, B^{\frac{1}{2}} h D u=\mathcal{O}\left(e^{-1 /(C h)}\right)
$$

in $L^{2}$-norm.

b) There exists a neighborhood $\Omega$ of 0 , where

$$
\begin{aligned}
u(x ; h) & =(a+r) e^{-\phi_{+}(x) / h}, \\
\|r\|_{L^{2}(\Omega)}, \quad\left\|B^{\frac{1}{2}} h D r\right\|_{L^{2}(\Omega)} & =\mathcal{O}\left(h^{\infty}\right) .
\end{aligned}
$$

Proof. Apply (9.26) with $v=u, z=\lambda(h), \epsilon=A h, A \gg 1$, to get

$$
h\left\|e^{\psi_{\epsilon} / h} u\right\|+h^{\frac{1}{2}}\left\|B^{\frac{1}{2}} h D\left(e^{\psi_{\epsilon} / h} u\right)\right\| \leq \mathcal{O}\left(h^{-N}\right), \quad N=N(A),
$$

where we also used that $e^{\psi_{\epsilon} / h}=\mathcal{O}\left(h^{-N}\right)$ in $\Omega_{G}\left(C_{0} \epsilon\right)$. Here, $\psi_{\epsilon}=f_{\delta_{0}}(\phi-\epsilon g(G))+$ $\mathcal{O}(\delta \epsilon)$ is larger than a positive constant outside any fixed neighborhood of 0 , so $u=\mathcal{O}\left(e^{-1 /(C h)}\right)$ in $L^{2}$-norm there. Moreover, since $\left\|e^{\psi_{\epsilon} / h} u\right\|=\mathcal{O}\left(h^{-N}\right)$, we have

$$
\mathcal{O}\left(h^{-N}\right) \geq h^{\frac{1}{2}}\left\|B^{\frac{1}{2}} h D\left(e^{\psi_{\epsilon} / h} u\right)\right\| \geq h^{\frac{1}{2}}\left\|e^{\psi_{\epsilon} / h} B^{\frac{1}{2}} h D u\right\|-\mathcal{O}\left(h^{\frac{1}{2}}\right)\left\|\left|\nabla \psi_{\epsilon}\right| e^{\psi_{\epsilon} / h} u\right\| .
$$

Here $\nabla \psi_{\epsilon}=\mathcal{O}(1)$ (as we shall see more in detail below), so $\left\|e^{\psi_{\epsilon} / h} B^{\frac{1}{2}} h D u\right\|=$ $\mathcal{O}\left(h^{-N-\frac{1}{2}}\right)$, so $B^{\frac{1}{2}} h D u=\mathcal{O}\left(e^{-\frac{1}{C h}}\right)$ in $L^{2}$-norm away from any given fixed neighborhood of 0 . The proof of a) is complete.

To prove b), we apply (9.26) to $v=u-\chi u_{\mathrm{WKB}}, z=\lambda(h)$ with $\epsilon=A h, A \gg 1$. Since $\left\|u-\chi u_{\mathrm{WKB}}\right\|=\mathcal{O}\left(h^{\infty}\right)$ by $(8.32)$, and $\left\|e^{\psi_{\epsilon} / h}(P-z) \chi u_{\mathrm{WKB}}\right\|=\mathcal{O}\left(h^{\infty}\right)$ if we 
arrange so that $\psi_{\epsilon}<\phi$ on supp $\nabla \chi$, we conclude that $\left\|e^{\psi_{\epsilon} / h}\left(u-\chi u_{\mathrm{WKB}}\right)\right\|_{\Omega_{G}\left(C_{0} \epsilon\right)}=$ $\mathcal{O}\left(h^{\infty}\right)$ and hence

$$
h\left\|e^{\psi_{\epsilon} / h}\left(u-\chi u_{\mathrm{WKB}}\right)\right\|+h^{\frac{1}{2}}\left\|B^{\frac{1}{2}} h D\left(e^{\psi_{\epsilon} / h}\left(u-\chi u_{\mathrm{WKB}}\right)\right)\right\| \leq \mathcal{O}\left(h^{\infty}\right) .
$$

In a small neighborhood of 0 , we have

$$
e^{\psi_{\epsilon} / h}=e^{\frac{\phi}{h}-\frac{\epsilon g(G)}{h}}=e^{-A g(G)} e^{\frac{\phi}{h}},
$$

where by (9.14)

$$
\begin{aligned}
& e^{-A g(G)}=\mathcal{O}(1) \epsilon^{-A} \quad \text { for } \quad G(x) \leq C_{0} \epsilon, \\
& e^{-A g(G)}=G(x)^{-A} \quad \text { for } \quad G(x) \geq C_{0} \epsilon .
\end{aligned}
$$

It then follows from $(9.28)$, that $u=(a+r) e^{-\phi_{+} / h}$ in a neighborhood $\Omega$ of 0 , with $\|r\|_{L^{2}(\Omega)}=\mathcal{O}\left(h^{\infty}\right)$.

To get the corresponding bound on $B^{\frac{1}{2}} h D r$, we just have to proceed as in the proof of a) and use that $\nabla \psi_{\epsilon}=\nabla \phi-\epsilon g^{\prime}(G) \nabla G$, where

$$
\begin{aligned}
& g^{\prime}(G) \nabla G=\mathcal{O}\left(\epsilon^{-\frac{1}{2}}\right) \quad \text { for } \quad G(x)<C_{0} \epsilon, \\
& g^{\prime}(G) \nabla G=\frac{\nabla G}{G}=\mathcal{O}\left(\epsilon^{-\frac{1}{2}}\right) \text { for } \quad G(x) \geq C_{0} \epsilon .
\end{aligned}
$$

Thus $\nabla \psi_{\epsilon}=\nabla \phi+\mathcal{O}(\sqrt{\epsilon})=\mathcal{O}(1)$ and we conclude that $\left\|B^{\frac{1}{2}} h D r\right\|_{L^{2}(\Omega)}=$ $\mathcal{O}\left(h^{\infty}\right)$.

Remark 9.2. If we drop the assumption (9.1) and allow $N-1$ more points $\rho_{2}, \ldots, \rho_{N}$ in $\mathcal{C}$, then Theorem 9.1 is still valid, provided that all $\mu_{j, k}$ in 8.24 with $j \geq 2$ are different from the value $\mu$, associated to $\rho_{1}=(0,0)$.

\section{Supersymmetric approach}

The Witten approach has been independently extended to the case of non-elliptic Operators like the Kramers-Fokker-Planck Operator in [24] (in supersymmetric language) and in [2] (in terms of differential forms). See also [17].

We start by a quick review of that in the semiclassical case, then we establish some basic facts about the principal and subprincipal symbols, especially at the critical points of the given weight function.

\subsection{Generalities}

Let

$$
A(x): T_{x}^{*} M \rightarrow T_{x} M, \quad x \in M,
$$

be an invertible map depending smoothly on $x \in M$. Then we have the real nondegenerate bilinear form

$$
\langle u \mid v\rangle_{A(x)}=\left\langle\wedge^{k} A(x)(u) \mid v\right\rangle, \quad u, v \in \wedge^{k} T_{x}^{*} M .
$$


If $a: \wedge^{k} T_{x}^{*} M \rightarrow \wedge^{\ell} T_{x}^{*} M$ is a linear map, we define the "adjoint" $a^{A, *}: \wedge^{\ell} T_{x}^{*} M \rightarrow$ $\wedge{ }^{k} T_{x}^{*} M$ by

$$
\langle a u \mid v\rangle_{A(x)}=\left\langle u \mid a^{A, *} v\right\rangle_{A(x)} .
$$

(In the complexified case, we use the sesquilinear scalar product $(u \mid v)_{A}=\langle u \mid \bar{v}\rangle_{A}$ and define $a^{A, *}$ the same way.)

If $\omega$ is a one form and $u$ and $v$ are $k-1$ and $k$ forms respectively, we get at $x$ :

$$
\langle\omega \wedge u \mid v\rangle_{A}=\left\langle A \omega \wedge\left(\wedge^{k-1} A\right) u \mid v\right\rangle=\left\langle\wedge^{k-1} A u \mid(A \omega)^{\rfloor} v\right\rangle=\left\langle u \mid(A \omega)^{\lrcorner} v\right\rangle_{A},
$$

so

$$
\left(\omega^{\wedge}\right)^{A, *}=(A \omega)^{\rfloor},
$$

where $\lrcorner$ denotes the usual Operator of contraction. Let $\mu(d x)$ be a locally finite measure with a smooth positive density. When $M=\mathbf{R}^{n}, \mu$ will be the Lebesgue measure. Sometimes we also use the symbol $\mu$ for the corresponding density.

If $u, v$ are smooth $k$ forms with $\operatorname{supp} u \cap \operatorname{supp} v$ compact, we define

$$
\langle u \mid v\rangle_{A}=\int\langle u(x) \mid v(x)\rangle_{A(x)} \mu(d x), \quad(u \mid v)_{A}=\int(u(x) \mid v(x))_{A(x)} \mu(d x)
$$

and denote by $a^{A, *}$ the formal adjoint of an Operator $a: C_{0}^{\infty}\left(M ; \wedge^{k} T^{*} M\right) \rightarrow$ $\mathcal{D}^{\prime}\left(M ; \wedge^{\ell} T^{*} M\right)$. If we fix some local coordinates $x_{1}, \ldots, x_{n}$ and write $\mu(d x)=$ $\mu(x) d x$ (by slight abuse of notation), we can consider

$$
\partial_{x_{j}}: C_{0}^{\infty}\left(M ; \wedge^{k} T^{*} M\right) \rightarrow C_{0}^{\infty}\left(M ; \wedge^{k} T^{*} M\right),
$$

acting coefficient-wise, and a straightforward computation shows that

$$
\begin{aligned}
\left(\partial_{x_{j}}\right)^{A, *} & =\left(\mu^{t}\left(\wedge^{k} A\right)\right)^{-1}\left(-\partial_{x_{j}}\right) \circ\left(\mu^{t}\left(\wedge^{k} A\right)\right) \\
& =-\partial_{x_{j}}-\frac{\partial_{x_{j}} \mu}{\mu}-{ }^{t}\left(\wedge^{k} A\right)^{-1 t}\left(\partial_{x_{j}}\left(\wedge^{k} A\right)\right) .
\end{aligned}
$$

We only retain that

$$
\left(h \partial_{x_{j}}\right)^{A, *}=-h \partial_{x_{j}}+\mathcal{O}(h),
$$

where $\mathcal{O}(h)$ stands for multiplication by a smooth matrix, which is $\mathcal{O}(h)$ with all its derivatives, uniformly on all of $M$ when $M=\mathbf{R}^{n}$, and which is $=0$ when $A(x)$, $\mu(x)$ are constant.

Let $\phi \in C^{\infty}(M ; \mathbf{R})$ and introduce the Witten (de Rham) complex

$$
\begin{aligned}
d_{\phi} & =e^{-\phi / h} \circ h d \circ e^{\phi / h} \\
& =h d+(d \phi)^{\wedge}: C_{0}^{\infty}\left(M ; \wedge^{k} T^{*} M\right) \rightarrow C_{0}^{\infty}\left(M ; \wedge^{k+1} T^{*} M\right),
\end{aligned}
$$

with $d_{\phi}^{2}=0$.

In local coordinates (always the canonical ones when $M=\mathbf{R}^{n}$ ) we have

$$
d_{\phi}=\sum_{1}^{n}\left(h \partial_{x_{j}}+\partial_{x_{j}} \phi\right) \circ d x_{j}^{\wedge},
$$


where $h \partial_{x_{j}}+\partial_{x_{j}} \phi$ acts coefficient-wise and commutes with $d x_{j}^{\wedge}$, so

$$
d_{\phi}^{A, *}=\sum_{1}^{n}\left(-h \partial_{x_{k}}+\partial_{x_{k}} \phi+\mathcal{O}(h)\right) \circ A\left(d x_{k}\right)^{\rfloor},
$$

where from now on, $\mathcal{O}(h)$ and $\mathcal{O}\left(h^{2}\right)$ will have the same meaning as after (10.7).

The corresponding Witten-Hodge Laplacian is given by

$$
-\Delta_{A}=d_{\phi}^{A, *} d_{\phi}+d_{\phi} d_{\phi}^{A, *} .
$$

Since

we also have

$$
\left(d_{\phi}^{A, *}\right)^{2}=\left(d_{\phi}^{2}\right)^{A, *}=0
$$

$$
-\Delta_{A}=\left(d_{\phi}+d_{\phi}^{A, *}\right)^{2},
$$

and $-\Delta_{A}$ conserves the degree of differential forms.

Choose local coordinates $x_{1}, \ldots, x_{n}$ (to be the standard ones when $M=\mathbf{R}^{n}$ ) and write

$$
A\left(d x_{k}\right)=\sum_{j} A_{j, k}(x) \partial_{x_{j}}, \quad A_{j, k}(x)=\left\langle A\left(d x_{k}\right) \mid d x_{j}\right\rangle .
$$

Let $Z_{j}=h \partial_{x_{j}}+\partial_{x_{j}} \phi, Z_{k}^{A, *}=h \partial_{x_{k}}^{A, *}+\partial_{x_{k}} \phi$. Notice that $\left[Z_{j}, d x_{k}^{\wedge}\right]=0$, so

$$
\left[Z_{k}^{A, *}, A\left(d x_{j}\right)^{\lrcorner}\right]=0 .
$$

Writing $d_{\phi}=\sum_{1}^{n} Z_{j} \circ d x_{j}^{\wedge}, d_{\phi}^{A, *}=\sum_{1}^{n} Z_{k}^{A, *} \circ A\left(d x_{k}\right)^{\lrcorner}$, we get

$$
-\Delta_{A}=\sum_{j, k}\left(Z_{k}^{A, *} \circ A\left(d x_{k}\right)^{\lrcorner} d x_{j}^{\wedge} Z_{j}+Z_{j} d x_{j}^{\wedge} A\left(d x_{k}\right)^{\rfloor} Z_{k}^{A, *}\right) .
$$

Here, we use the general identity $\nu^{\wedge} \mu^{\lrcorner}+\mu^{\lrcorner} \nu^{\wedge}=\langle\nu, \mu\rangle 1$ on the first term in the parenthesis to get

$$
\begin{aligned}
-\Delta_{A} & =\mathrm{I}+\mathrm{II}+\mathrm{III}, \\
\mathrm{I} & =\sum_{j, k} Z_{k}^{A, *} A_{j, k} Z_{j}, \\
\mathrm{II} & =-\sum_{j, k} Z_{k}^{A, *} d x_{j}^{\wedge} A\left(d x_{k}\right)^{\rfloor} Z_{j} \\
\mathrm{III} & =\sum_{j, k} d x_{j}^{\wedge} Z_{j} Z_{k}^{A, *} A\left(d x_{k}\right)^{\rfloor},
\end{aligned}
$$

where $A_{j, k}=\left\langle d x_{j}, A\left(d x_{k}\right)\right\rangle$. We have

$$
\left[Z_{j}, A\left(d x_{k}\right)^{\lrcorner}\right]=h\left(\left(\partial_{x_{j}} A\right)\left(d x_{k}\right)\right)^{\lrcorner} .
$$

Using the identity $\left(U^{A, *}\right)^{t} A, *=U$ (see Subsection 10.4), we see that

$$
\left[Z_{k}^{A, *}, d x_{j}^{\wedge}\right]^{t} A, *=\left[\left({ }^{t} A\left(d x_{j}\right)\right)^{\lrcorner}, Z_{k}\right]=-h\left(\left(\partial_{x_{k}}{ }^{t} A\right)\left(d x_{j}\right)\right)^{\lrcorner},
$$


hence

$$
\left[Z_{k}^{A, *}, d x_{j}^{\wedge}\right]=-h\left({ }^{t} A^{-1} \partial_{x_{k}}{ }^{t} A\left(d x_{j}\right)\right)^{\wedge}
$$

Using these commutator relations, we move $d x_{j}^{\wedge}$ to the left and $A\left(d x_{k}\right)^{\rfloor}$to the right in II and combining with III, we finally obtain that

$$
\begin{aligned}
-\Delta_{A}= & \sum_{j, k} Z_{k}^{A, *} A_{j, k} Z_{j}+\sum_{j, k} d x_{j}^{\wedge}\left[Z_{j}, Z_{k}^{A, *}\right] A\left(d x_{k}\right)^{\lrcorner} \\
& +h \sum_{j, k} Z_{k}^{A, *} d x_{j}^{\wedge}\left(\left(\partial_{x_{j}} A\right)\left(d x_{k}\right)\right)^{\lrcorner}+h \sum_{j, k}\left({ }^{t} A^{-1}\left(\partial_{x_{k}}{ }^{t} A\right) d x_{j}\right)^{\wedge} A\left(d x_{k}\right)^{\lrcorner} Z_{j} \\
& +h^{2} \sum_{j, k}\left({ }^{t} A^{-1}\left(\partial_{x_{k}}{ }^{t} A\right) d x_{j}\right)^{\wedge}\left(\left(\partial_{x_{j}} A\right) d x_{k}\right)^{\lrcorner} .
\end{aligned}
$$

Modulo $\mathcal{O}(h)\left(h \partial_{x}+\partial_{x} \phi\right)+\mathcal{O}(h)\left(-h \partial_{x}+\partial_{x} \phi\right)+\mathcal{O}\left(h^{2}\right)$, we get

$$
\begin{aligned}
-\Delta_{A} \equiv & \sum_{j, k}\left(-h \partial_{x_{k}}+\partial_{x_{k}} \phi\right) A_{j, k}(x)\left(h \partial_{x_{j}}+\partial_{x_{j}} \phi\right) \\
& +\sum_{j, k} 2 h \partial_{x_{j}} \partial_{x_{k}} \phi \circ d x_{j}^{\wedge} A\left(d x_{k}\right)^{\rfloor},
\end{aligned}
$$

where the error terms vanish when $A(x)$ and $\mu(x)$ are constant (for the chosen coordinates).

Now write

$$
A(x)=B(x)+C(x), \quad{ }^{t} B(x)=B(x), \quad{ }^{t} C(x)=-C(x) .
$$

Then (10.13) gives

$$
\begin{aligned}
-\Delta_{A} \equiv & \sum_{j, k}\left(-h \partial_{x_{k}}+\partial_{x_{k}} \phi\right) B_{j, k}(x)\left(h \partial_{x_{j}}+\partial_{x_{j}} \phi\right) \\
& +\sum_{j, k}\left(\left(\partial_{x_{k}} \phi\right) C_{j, k} h \partial_{x_{j}}+h \partial_{x_{j}} \circ C_{j, k} \circ\left(\partial_{x_{k}} \phi\right)\right)-\sum_{j, k} h \partial_{x_{k}}\left(C_{j, k}\right) h \partial_{x_{j}} \\
& +\sum_{j, k} 2 h \partial_{x_{j}} \partial_{x_{k}} \phi \circ d x_{j}^{\wedge} A\left(d x_{k}\right)^{\rfloor} .
\end{aligned}
$$

Again, this becomes an equality when $A, \mu$ are constant. Note that the last term vanishes on 0 -forms, i.e., on scalar functions. To recover the Kramers-FokkerPlanck Operator (cf. [24]), replace $n$ by $2 n$, put $M=\mathbf{R}_{x, y}^{2 n}$,

$$
A=\frac{1}{2}\left(\begin{array}{cc}
0 & 1 \\
-1 & \gamma
\end{array}\right)
$$


and let $\mu=d x d y$ be the Lebesgue measure. Then (10.15) is an equality and we get for 0 -forms:

$$
\begin{aligned}
-\Delta_{A}^{(0)}= & \frac{\gamma}{2} \sum_{j=1}^{n}\left(-h \partial_{y_{j}}+\partial_{y_{j}} \phi\right)\left(h \partial_{y_{j}}+\partial_{y_{j}} \phi\right) \\
& +\frac{1}{2} \sum_{j}\left[\left(\partial_{y_{j}} \phi\right) h \partial_{x_{j}}-\left(\partial_{x_{j}} \phi\right) h \partial_{y_{j}}+h \partial_{x_{j}} \circ \partial_{y_{j}} \phi-h \partial_{y_{j}} \circ \partial_{x_{j}} \phi\right] \\
= & \frac{\gamma}{2} \sum_{j=1}^{n}\left(-h \partial_{y_{j}}+\partial_{y_{j}} \phi\right)\left(h \partial_{y_{j}}+\partial_{y_{j}} \phi\right)+h H_{\phi},
\end{aligned}
$$

where

$$
H_{\phi}=\sum\left(\partial_{y_{k}} \phi \partial_{x_{k}}-\partial_{x_{k}} \phi \partial_{y_{k}}\right)
$$

is the Hamilton field of $\phi$ with respect to the standard symplectic form $\sum d y_{j} \wedge d x_{j}$. If we choose

$$
\phi(x, y)=\frac{1}{2} y^{2}+V(x),
$$

we get the Kramers-Fokker-Planck Operator

$$
-\Delta_{A}^{(0)}=y \cdot h \partial_{x}-V^{\prime}(x) \cdot h \partial_{y}+\frac{\gamma}{2}\left(-h \partial_{y}+y\right) \cdot\left(h \partial_{y}+y\right) .
$$

10.2. The principal symbol of the Hodge Laplacian

The principal symbol of $-\Delta_{A}$ in the sense of $h$-differential operators is scalar and given by

$$
\begin{aligned}
p(x, \xi) & =\sum_{j, k} A_{j, k}\left(-i \xi_{k}+\partial_{x_{k}} \phi\right)\left(i \xi_{j}+\partial_{x_{j}} \phi\right) \\
& =\sum_{j, k} B_{j, k}\left(\xi_{j} \xi_{k}+\partial_{x_{j}} \phi \partial_{x_{k}} \phi\right)+2 i \sum_{j, k} C_{j, k} \partial_{x_{k}} \phi \xi_{j} .
\end{aligned}
$$

The corresponding real symbol $q(x, \xi)=-p(x, i \xi)$ is given by

$$
\begin{aligned}
q(x, \xi) & =\sum_{j, k} A_{j, k}\left(\xi_{k}+\partial_{x_{k}} \phi\right)\left(\xi_{j}-\partial_{x_{j}} \phi\right) \\
& =\sum_{j, k} B_{j, k}\left(\xi_{j} \xi_{k}-\partial_{x_{j}} \phi \partial_{x_{k}} \phi\right)+2 \sum_{j, k} C_{j, k} \partial_{x_{k}} \phi \xi_{j} .
\end{aligned}
$$

It vanishes on the two Lagrangian manifolds $\Lambda_{ \pm \phi}$.

We define

$$
\nu_{ \pm}=H_{\left.\right|_{\Lambda_{ \pm \phi}}} .
$$

Using $x_{1}, \ldots, x_{n}$ as coordinates on $\Lambda_{ \pm \phi}$, we get

$$
\begin{aligned}
& \nu_{+}=2 \sum_{j, k} A_{j, k} \partial_{x_{k}} \phi \partial_{x_{j}}=2 A(x)\left(\phi^{\prime}(x)\right) \cdot \partial_{x} \\
& \nu_{-}=-2 \sum_{j, k} A_{j, k} \partial_{x_{j}} \phi \partial_{x_{k}}=-2{ }^{t} A(x)\left(\phi^{\prime}(x)\right) \cdot \partial_{x} .
\end{aligned}
$$


(Even more radically, we could say that $\nu_{+}=2 A(x)(d \phi(x))$, where $A(x)$ is viewed as a map $T_{x}^{*} M \rightarrow T_{x} M$, and similarly for $\nu_{-}$.)

Let $x_{0}$ be a non-degenerate critical point of $\phi$, so that $\Lambda_{\phi}$ and $\Lambda_{-\phi}$ intersect transversally at $\left(x_{0}, 0\right)$. The spectrum of the linearization $F_{q}$ of $H_{q}$ at $\left(x_{0}, 0\right)$ is equal to the union of the spectra of the linearizations

$$
\nu_{+}^{0}=\left(2 A\left(x_{0}\right) \phi^{\prime \prime}\left(x_{0}\right) x\right) \cdot \partial_{x} \quad \text { and } \quad \nu_{-}^{0}=-\left(2^{t} A\left(x_{0}\right) \phi^{\prime \prime}\left(x_{0}\right) x\right) \cdot \partial_{x}
$$

of $\nu_{+}$and $\nu_{-}$respectively at $x_{0}$. Thus we are interested in the eigenvalues of the matrices $A \phi^{\prime \prime},{ }^{t} A \phi^{\prime \prime}$, where we write $A=A\left(x_{0}\right)$, and $\phi^{\prime \prime}=\phi^{\prime \prime}\left(x_{0}\right)$ for short. Here, we notice that ${ }^{t} A \phi^{\prime \prime}=\phi^{\prime \prime-1}\left(A \phi^{\prime \prime}\right) \phi^{\prime \prime}$ has the same eigenvalues as $A \phi^{\prime \prime}$ and similarly $\phi^{\prime \prime} A, \phi^{\prime \prime} A$ are isospectral to $A \phi^{\prime \prime}$. Thus

The eigenvalues of $F_{q}$ are given by $\pm 2 \lambda_{j}$,

$$
\text { where } \lambda_{1}, \ldots, \lambda_{n} \text { are the eigenvalues of } A \phi^{\prime \prime} \text {. }
$$

From questions about hypoellipticity (see [22]) we would like to know when all the eigenvalues of $F_{p}$ avoid the real axis, or equivalently, when all the eigenvalues of $F_{q}$ (the linearization of $H_{q}$ at $\left(x_{0}, 0\right)$ ) avoid the imaginary axis.

We assume from now on that

$$
B(x) \geq 0, \quad x \in M .
$$

Then, if $\phi^{0}(x)=\frac{1}{2} \phi^{\prime \prime} x \cdot x$ is the Hessian quadratic form of $\phi$ at $x_{0}$, we have

$$
\nu_{+}^{0}\left(\phi^{0}\right)=\left\langle 2 B \phi^{\prime \prime} x, \phi^{\prime \prime} x\right\rangle \geq 0 .
$$

Lemma 10.1. Let $\mu\left(x, \partial_{x}\right)=M x \cdot \partial_{x}$ be a real linear vector field on $\mathbf{R}^{n}$. Let $n_{ \pm} \in$ $\mathbf{N}, n_{+}+n_{-}=n$. Then the following two statements are equivalent:

(A) $M$ has $n_{+}$eigenvalues with real part $>0$ and $n_{-}$eigenvalues with real part $<0$.

(B) There exists a quadratic form $G: \mathbf{R}^{n} \rightarrow \mathbf{R}$ of signature $\left(n_{+}, n_{-}\right)$and a constant $C>0$, such that

$$
\mu\left(x, \partial_{x}\right)(G) \geq \frac{1}{C}|x|^{2}, \quad x \in \mathbf{R}^{n} .
$$

Proof. Assume first that $\left(n_{+}, n_{-}\right)=(n, 0)$. If $(\mathrm{A})$ holds, we know that

$$
\left\|e^{t M} x\right\|^{2} \geq \frac{1}{C} e^{t / C}\|x\|^{2}, \quad t \geq 0
$$

for some constant $C>0$, and we can put (by a classical argument)

$$
G(x)=G_{T}(x)=\frac{1}{T} \int_{0}^{T}\left\|e^{t M} x\right\|^{2} d t, \quad T \gg 1 .
$$


Then

$$
\begin{aligned}
M x \cdot \partial_{x}\left(G_{T}(x)\right) & =\frac{1}{T} \int_{0}^{T} M x \cdot \partial_{x}\left\|e^{t M} x\right\|^{2} d t=\frac{1}{T} \int_{0}^{T} \frac{d}{d t}\left\|e^{t M} x\right\|^{2} d t \\
& =\frac{1}{T}\left(\left\|e^{T M} x\right\|^{2}-\|x\|^{2}\right) \geq \frac{1}{T}\left(\frac{1}{C} e^{T / C}-1\right)\|x\|^{2} \geq \frac{1}{2 T}\|x\|^{2},
\end{aligned}
$$

if $T$ is large enough. Thus we get (B).

Conversely, if (B) holds, we have with a new constant $C>0$, that

$$
\mu\left(x, \partial_{x}\right) G \geq \frac{1}{C} G,
$$

and hence

So

$$
\frac{d}{d t} G\left(e^{t M} x\right) \geq \frac{1}{C} G\left(e^{t M} x\right)
$$

$$
G\left(e^{t M} x\right) \geq e^{t / C} G(x), \quad t \geq 0 .
$$

Thus $\left\|e^{t M} x\right\| \geq C^{-1} e^{t / C}\|x\|$ for some new positive constant $C$ and we conclude that the eigenvalues of $M$ all have positive real parts.

Now consider the case of general $\left(n_{+}, n_{-}\right)$and assume first that $(\mathrm{A})$ holds. Then we have the $M$-invariant decomposition, $\mathbf{R}^{n}=L_{+} \oplus L_{+}$where $\operatorname{dim}\left(L_{ \pm}\right)=$ $n_{ \pm}$and $\sigma\left(M_{\left.\right|_{L_{+}}}\right)$belongs to the open right half plane in the + case and to the open left half plane in the - case. Hence we have positive definite quadratic forms $G_{ \pm}$on $L_{ \pm}$such that

$$
\pm M x \cdot \partial_{x}\left(G_{ \pm}\right) \geq \frac{1}{C}|x|^{2}, \quad x \in L_{ \pm} .
$$

Then $G=G_{+} \oplus\left(-G_{-}\right)$(defined in the obvious way) has the required properties in $(\mathrm{B})$.

Conversely, assume that (B) holds. Let $\widetilde{L}_{+}$be an $n_{+}$-dimensional subspace on which $G$ is positive definite. By (10.28), we have for all $x \in \mathbf{R}^{n}$,

$$
\mu\left(x, \partial_{x}\right) G(x) \geq \frac{1}{C} G(x), \quad C>0
$$

so if $x \in \widetilde{L}_{+}$, we get

$$
G\left(e^{t M} x\right) \geq e^{t / C} G(x), \quad t \geq 0
$$

and hence with a new constant $C>0$,

$$
\left|e^{t M} x\right| \geq \frac{1}{C} e^{t / C}|x|, \quad t \geq 0 .
$$

Similarly, if $\operatorname{dim}\left(\widetilde{L}_{-}\right)=n_{-}$and $G$ is negative definite on $\widetilde{L}_{-}$, we get

$$
\left|e^{t M} x\right| \geq \frac{1}{C} e^{|t| / C}|x|, \quad x \in \widetilde{L}_{-}, \quad t \leq 0 .
$$

Now we have the $M$-invariant decomposition

$$
\mathbf{R}^{n}=L_{+} \oplus L_{0} \oplus L_{-}
$$


where $L_{+}^{\mathbf{C}}, L_{0}^{\mathbf{C}}, L_{-}^{\mathbf{C}}$ are the sums of generalized eigenspaces of $M$ corresponding to the eigenvalues with real parts $>0,=0$ and $<0$ respectively. We see that necessarily, $\widetilde{L}_{+} \cap\left(L_{0} \oplus L_{-}\right)=0$, so

$$
n_{+}=\operatorname{dim} \widetilde{L}_{+} \leq \operatorname{dim} L_{+} .
$$

Similarly,

$$
n_{-}=\operatorname{dim} \widetilde{L}_{-} \leq \operatorname{dim} L_{-},
$$

so $\operatorname{dim} L_{+}=n_{+}, \operatorname{dim} L_{-}=n_{-}, \operatorname{dim} L_{0}=0$, and (A) follows.

If $B>0$, then (10.27) with strict inequality for $x \neq 0$, and Lemma 10.1 imply that $\nu_{ \pm}^{0}$ has $n_{ \pm}$eigenvalues with \pm real part $>0$, where $\left(n_{+}, n_{-}\right)$is the signature of $\phi^{\prime \prime}\left(x_{0}\right)$. It follows in that case that $F_{p}$ has no real eigenvalues. This last conclusion also follows from [22]. Indeed, in that case the quadratic approximation $p^{0}(x, \xi)$ of $p$ at $\left(x_{0}, 0\right)$ is elliptic in the sense that $\left|p^{0}(x, \xi)\right| \backsim|x|^{2}+|\xi|^{2}$ and takes its values in an angle $-\frac{\pi}{2}+\epsilon \leq \arg p^{0} \leq \frac{\pi}{2}-\epsilon$ for some $\epsilon>0$.

Now return to the general case, when we only assume (10.26) and $\phi^{\prime \prime}=\phi^{\prime \prime}\left(x_{0}\right)$ is non-degenerate of signature $\left(n_{+}, n_{-}\right)$. $\left(x_{0}, 0\right)$.

We next make some remarks about the quadratic approximation $p^{0}$ of $p$ at

Proposition 10.2. $\quad$ a) Assume that the matrix $A \phi^{\prime \prime}$ of $\nu_{+}^{0}$ has $m_{ \pm}$eigenvalues with \pm real part $>0, m_{+}+m_{-}=n$. Then there exists a real quadratic form $\mathcal{G}(x, \xi)$ on $\mathbf{R}^{2 n}$ such that

$$
\operatorname{Re} p^{0}\left((x, \xi)+i \epsilon H_{\mathcal{G}}(x, \xi)\right) \geq \frac{\epsilon}{C}|(x, \xi)|^{2}, \quad(x, \xi) \in \mathbf{R}^{2 n}, \quad 0<\epsilon \ll 1 .
$$

b) Conversely, assume that there exists a quadratic form $\mathcal{G}$ such that (10.29) holds. Then $A \phi^{\prime \prime}$ has $n_{ \pm}$eigenvalues with \pm real part $>0$, where $\left(n_{+}, n_{-}\right)$is the signature of $\phi^{\prime \prime}(0)$.

Recall that the condition (4.21) implies the existence of $\mathcal{G}$ as in a) of the proposition. (The converse is not true however. It is easy to find examples of purely imaginary quadratic forms $p^{0}$ for which there exist $\mathcal{G}$ as in a) of the proposition.)

Proof. a) Choose $\mathcal{G}(x, \xi)$ of the form $G(x)+\widetilde{G}(\xi)$, so that $H_{\mathcal{G}}(x, \xi)=\left(\widetilde{G}_{\xi}^{\prime},-G_{x}^{\prime}\right)$. Recall that $p^{0}$ is the quadratic approximation of $p$ at $\left(x_{0}, 0\right)$, obtained from (10.19) by freezing $A_{j, k}$ at $x_{0}$ and replacing $\partial_{x} \phi$ by $\phi^{\prime \prime} x=\left(\phi^{0}\right)^{\prime}(x), \phi^{\prime \prime}=\phi^{\prime \prime}\left(x_{0}\right)$.

We get

$$
\begin{aligned}
\operatorname{Re} p_{\epsilon}^{0}(x, \xi)= & \sum_{j, k} B_{j, k}\left(\xi_{j} \xi_{k}+\partial_{x_{j}} \phi^{0} \partial_{x_{k}} \phi^{0}\right) \\
& +2 \epsilon\left(\sum_{j, k} C_{j, k}\left(\partial_{x_{k}} \phi^{0}\right) \partial_{x_{j}} G-\sum_{j, k} C_{j, k}\left(\phi^{\prime \prime} \partial_{\xi} \widetilde{G}\right)_{k} \xi_{j}\right)+\mathcal{O}\left(\epsilon^{2}|(x, \xi)|^{2}\right),
\end{aligned}
$$


so it suffices to have

$$
\begin{aligned}
& C \phi^{\prime \prime} x \cdot \partial_{x} G \geq \frac{1}{C}|x|^{2} \quad \text { on } \quad \phi^{\prime \prime-1} \mathcal{N}(B), \\
& \phi^{\prime \prime} C \xi \cdot \partial_{\xi} \widetilde{G} \geq \frac{1}{C}|\xi|^{2} \quad \text { on } \quad \mathcal{N}(B),
\end{aligned}
$$

where we also used the antisymmetry of $C$ in the last equation. This would follow from

$$
A \phi^{\prime \prime} x \cdot \partial_{x} G \geq \frac{1}{C}|x|^{2}, \quad \phi^{\prime \prime} A \xi \cdot \partial_{\xi} \widetilde{G} \geq \frac{1}{C}|\xi|^{2}, \quad x, \xi \in \mathbf{R}^{n},
$$

and in order to find such functions $G$ and $\widetilde{G}$, it suffices to apply Lemma 10.1 to the isospectral matrices $A \phi^{\prime \prime}$ and $\phi^{\prime \prime} A$.

b) Let $\mathcal{G}$ be as in (10.29). The quadratic form

$$
p_{\epsilon}^{0}(x, \xi)=p^{0}\left(\exp i \epsilon H_{\mathcal{G}}(x, \xi)\right)=p^{0}(x, \xi)+i \epsilon H_{\mathcal{G}} p^{0}+\mathcal{O}\left(\epsilon^{2}\right)
$$

is elliptic on the real phase space and takes its values in an angle

$$
e^{i\left[-\frac{\pi}{2}+\frac{\epsilon}{C}, \frac{\pi}{2}-\frac{\epsilon}{C}\right]}[0,+\infty[
$$

so we know from [22] that $F_{p_{\epsilon}^{0}}$ has no real eigenvalues.

On the other hand $p_{\epsilon}^{0}$ and $p^{0}$ are related by a canonical transformation, so $F_{p_{\epsilon}^{0}}$ and $F_{p_{0}}$ are isospectral. Hence $A \phi^{\prime \prime}$ has $m_{ \pm}$eigenvalues with \pm real part $>0$, where $m_{+}+m_{-}=n$. To see that $m_{ \pm}=n_{ \pm}$, we just replace $B$ by $B+\delta 1,0<\delta \ll 1$, to reduce ourselves to the elliptic case, and apply the observation after the proof of Lemma 10.1.

\subsection{The subprincipal symbol}

We next look at the subprincipal term in (10.13). Write

$$
A\left(d x_{k}\right)=\sum_{\nu} A_{\nu, k} \partial_{x_{\nu}}, \quad A\left(d x_{k}\right)^{\rfloor}=\sum_{\nu} A_{\nu, k} \partial_{x_{\nu}}^{\lrcorner},
$$

so the second sum in (10.13) becomes

$$
2 h \sum_{j, k, \nu} \phi_{j, k}^{\prime \prime} A_{\nu, k} \circ d x_{j}^{\wedge} \partial_{x_{\nu}}^{\rfloor}=2 h \sum_{j, \nu}\left(\phi^{\prime \prime} \circ{ }^{t} A\right)_{j, \nu} \circ d x_{j}^{\wedge} \partial_{x_{\nu}}^{\rfloor}
$$

which simplifies further to

$$
2 h \sum_{j}\left(\phi^{\prime \prime} \circ{ }^{t} A\right)\left(d x_{j}\right)^{\wedge} \partial_{x_{j}}^{\rfloor} .
$$

Now we restrict the attention to a non-degenerate critical point $x_{0}$ of $\phi$ and we shall compute the subprincipal symbol of $-\Delta_{A}$ at the corresponding doubly characteristic point $\left(x_{0}, 0\right)$. At that point $\phi^{\prime \prime} \circ{ }^{t} A: T_{x_{0}}^{*} M \rightarrow T_{x_{0}}^{*} M$ is invariantly defined and it is easy to check that (10.32) is also invariantly defined: we get the same quantity if we replace $d x_{1}, \ldots, d x_{n}, \partial_{x_{1}}, \ldots, \partial_{x_{n}}$, by $\omega_{1}, \ldots, \omega_{n}, \omega_{1}^{*}, \ldots, \omega_{n}^{*}$, where $\omega_{1}, \ldots, \omega_{n}$ is any basis in the complexified cotangent space and $\omega_{1}^{*}, \ldots, \omega_{n}^{*}$ is the dual basis of tangent vectors for the natural bilinear pairing. 
Assume that the equivalent conditions of Proposition 10.2 hold and denote the corresponding eigenvalues (that are also the eigenvalues of $\phi^{\prime \prime} \circ^{t} A$ ) by $\lambda_{1}, \ldots, \lambda_{n}$ with $\operatorname{Re} \lambda_{j}>0$ for $1 \leq j \leq n_{+}$and with $\operatorname{Re} \lambda_{j}<0$ for $n_{+}+1 \leq j \leq n=n_{+}+n_{-}$. The eigenvalues of $F_{p}$ are then $\pm 2 i \lambda_{j}$ (in view of (10.25) and the isospectrality of $F_{p}$ and $i F_{q}$ reviewed prior to Lemma 8.1), so

$$
\widetilde{\operatorname{tr}} F_{p}:=\frac{1}{i} \sum_{\substack{\mu \in \sigma\left(F_{p}\right) \\ \operatorname{Im} \mu>0}} \mu=\sum_{1}^{n_{+}} 2 \lambda_{j}-\sum_{n_{+}+1}^{n} 2 \lambda_{j} .
$$

The subprincipal symbol of the first term in $(10.13)\left(\right.$ at $\left.\left(x_{0}, 0\right)\right)$ is equal to

$$
\begin{aligned}
\sum_{j, k} A_{j, k} \frac{1}{2 i}\left\{-i \xi_{k}+\partial_{x_{k}} \phi, i \xi_{j}+\partial_{x_{j}} \phi\right\} & =-\sum_{j, k} A_{j, k} \phi_{j, k}^{\prime \prime} \\
& =-\operatorname{tr}\left(A \phi^{\prime \prime}\right)=-\sum_{1}^{n} \lambda_{j} .
\end{aligned}
$$

The eigenvalues of $\sum_{j}\left(\phi^{\prime \prime} \circ{ }^{t} A\right)\left(d x_{j}\right)^{\wedge} \partial_{x_{j}}^{\rfloor}$on the space of $m$-forms are easily calculated, if we replace $d x_{1}, \ldots, d x_{n}$ by a basis of eigenvectors $\omega_{1}, \ldots, \omega_{n}$ of $\phi^{\prime \prime} t A$, so that

$$
\left(\phi^{\prime \prime} \circ{ }^{t} A\right)\left(\omega_{j}\right)=\lambda_{j} \omega_{j}
$$

and $\partial_{x_{j}}$ by the corresponding dual basis vectors $\omega_{j}^{*}$. (Here we assume to start with that there are no Jordan blocks. This can be achieved by an arbitrarily small perturbation of $A$, and we can extend the end result of our calculation to the general case by continuity.) We get

$$
\sum_{j}\left(\phi^{\prime \prime} \circ{ }^{t} A\right)\left(d x_{j}\right)^{\wedge} \partial_{x_{j}}^{\rfloor}=\sum_{j} \lambda_{j} \omega_{j}^{\wedge} \omega_{j}^{*\rfloor} .
$$

A basis of eigenforms of this Operator is given by $\omega_{j_{1}} \wedge \cdots \wedge \omega_{j_{m}}, 1 \leq j_{1}<j_{2}<$ $\cdots<j_{m} \leq n$ and the corresponding eigenvalues are $\lambda_{j_{1}}+\cdots+\lambda_{j_{m}}$.

Let $S_{P}$ be the subprincipal symbol of $(10.13)$ at $\left(x_{0}, 0\right)$. Then the eigenvalues of

$$
\frac{1}{2} \tilde{\operatorname{tr}} F_{p}+S_{P}, \quad \text { acting on } m \text { forms }
$$

are

$$
\begin{array}{r}
\sum_{1}^{n_{+}} \lambda_{j}-\sum_{n_{+}+1}^{n} \lambda_{j}-\sum_{1}^{n} \lambda_{j}+2\left(\lambda_{j_{1}}+\cdots+\lambda_{j_{m}}\right)=2\left(\lambda_{j_{1}}+\cdots+\lambda_{j_{m}}-\sum_{n_{+}+1}^{n} \lambda_{j}\right), \\
1 \leq j_{1}<\cdots<j_{m} \leq n .
\end{array}
$$

We conclude that if $m \neq n_{-}$, then all the eigenvalues have a real part $>0$ and if $m=n_{-}$, then precisely one eigenvalue is equal to 0 , while the others have positive real part. 


\subsection{A symmetry for adjoints}

Our last remark in this section concerns symmetry relations for the $A$, $*$ adjoints. If $D: L^{2}\left(\Omega ; \wedge^{k} T^{*} \Omega\right) \rightarrow L^{2}\left(\Omega ; \wedge^{j} T^{*} \Omega\right)$, then a simple calculation shows that

$$
D^{A, *}=\left(\wedge^{k}\left({ }^{t} A\right)\right)^{-1} D^{*}\left(\wedge^{j}\left({ }^{t} A\right)\right),
$$

where $D^{*}$ denotes the adjoint with respect to the measure $\mu$.

We also have

$$
(u \mid v)_{A}={\overline{(v \mid u)_{t}}} .
$$

Playing with these relations we see that

$$
D=\left(D^{A, *}\right)^{t} A, * \text {. }
$$

This can be applied to $-\Delta_{A}$ and we get

$$
\left(-\Delta_{A}\right)^{t} A, *=-\Delta_{t} \text {. }
$$

\section{The double well case}

In this section we assume that

$$
M=\mathbf{R}^{n}, \quad A=A(x) \text { is independent of } x, \text { and invertible. }
$$

We decompose $A$ as in (10.14) and assume (10.26). Let $\phi \in C^{\infty}\left(\mathbf{R}^{n} ; \mathbf{R}\right)$ be such that

$$
\partial_{x}^{\alpha} \phi(x)=\mathcal{O}(1), \quad \partial_{x}^{\alpha}\left(\left\langle B \partial_{x} \phi, \partial_{x} \phi\right\rangle\right)=\mathcal{O}(1), \quad|\alpha| \geq 2 .
$$

Consider $P^{(q)}=-\Delta_{A}^{(q)}$ which according to (10.15) becomes

$$
\begin{aligned}
P^{(q)}= & \sum_{j, k} h D_{x_{j}} B_{j, k} h D_{x_{k}}+\sum_{j, k}\left(\partial_{x_{j}} \phi\right) B_{j, k}\left(\partial_{x_{k}} \phi\right)-h \operatorname{tr}\left(B \phi^{\prime \prime}\right) \\
& +\sum_{j, k}\left(\left(\partial_{x_{k}} \phi\right) C_{j, k} h \partial_{x_{j}}+h \partial_{x_{j}} \circ C_{j, k}\left(\partial_{x_{k}} \phi\right)\right) \\
& +2 h \sum_{j, k}\left(\partial_{x_{j}} \partial_{x_{k}} \phi\right) d x_{j}^{\wedge} A\left(d x_{k}\right)^{\rfloor} .
\end{aligned}
$$

Apart from the third and the last terms which are $\mathcal{O}(h)$ with all their derivatives, this is of the form (2.1) with $b_{j, k}=B_{j, k}, c_{j}(x)=\sum_{k} C_{j, k} \partial_{x_{k}} \phi, p_{0}(x)=$ $\left\langle B \partial_{x} \phi, \partial_{x} \phi\right\rangle$. We define $p_{2}, p_{1}, p_{0}$ as in Section 2 and see that $(2.4)-(2.7)$ hold.

Assume that

$\phi$ is a Morse function with critical points $x_{1}, \ldots, x_{N} \in \mathbf{R}^{n}$,

$$
\left|\phi^{\prime}(x)\right| \geq 1 / C, \quad|x| \geq C .
$$

Let $\mathcal{C}=\left\{\rho_{j} ; j=1, \ldots, N\right\}$ where $\rho_{j}=\left(x_{j}, 0\right) \in T^{*} \mathbf{R}^{n}$. Then $\rho_{j}$ are critical points with critical value 0 for $p_{2}, p_{1}, p_{0}$. Since $A$ is invertible, $\mathcal{N}(B) \cap \mathcal{N}(C)=0$, and hence (4.4) holds. We adopt the dynamical assumptions (4.21), (4.22) (or equivalently $(4.21),(4.24))$ and $(4.23)$. Then we can apply the results of Sections 8,9 to $P^{(q)}$ since neither the presence of the bounded subprincipal symbol in (11.3) nor 
the non-scalar nature of the Operators has any serious influence. In the preceding section we saw that we are in the case when the conditions of Proposition 10.2 are fulfilled. The only change in Theorem 8.3 is that the $\mu_{j, k}$ in (8.24) are of the form

$$
\mu_{j, k}=\frac{1}{i} \sum_{l=1}^{n} \nu_{j, k, \ell} \lambda_{\ell}+\frac{1}{2} \widetilde{\operatorname{tr}}\left(F_{p}\left(\rho_{j}\right)\right)+\gamma_{j, k},
$$

where $\gamma_{j, k}$ is any eigenvalue of the subprincipal symbol $S_{P^{(q)}}$ at $\left(x_{j}, 0\right)$. From the calculations in Subsection 10.3 we notice that the $\mu_{j, k}$ will be confined to a sector $\{0\} \cup\{|\arg z|<\pi / 2-1 / C\}$ around $\left[0,+\infty\left[\right.\right.$ and it is precisely when $x_{j}$ is of index $q$ (i.e., when the Hessian of $\phi$ at $x_{j}$ has precisely $q$ negative eigenvalues) that one of the $\mu_{j, k}$ may be equal to 0 .

We now add more specific conditions for the double well case. Assume that

$\phi$ has precisely three critical points, two local

$$
\text { minima } U_{ \pm 1} \text {, and a "saddle point" } U_{0} \text { of index one. }
$$

Then $\phi(x) \rightarrow+\infty$ with $\phi(x) \geq \frac{1}{C}|x|$, for $|x| \geq C$.

Put $S_{j}=\phi\left(U_{0}\right)-\phi\left(U_{j}\right), j= \pm 1$, so that $S_{j}>0$. The set $\phi^{-1}(]-\infty, \phi\left(U_{0}\right)[)$ has precisely two connected components $D_{j}, j= \pm 1$, determined by the condition $U_{j} \in D_{j}$. Under these assumptions we know that $P^{(0)}=-\Delta_{A}^{(0)}$ has precisely two eigenvalues $\mu_{0}, \mu_{1}=o(h)$ spanning a corresponding 2-dimensional spectral subspace $E^{(0)}$. Actually one of these two eigenvalues, say $\mu_{0}$, is equal to 0 with $e^{-\phi / h}$ as the corresponding eigenfunction and since a truncation of this function can be used as a quasimode near each of $U_{ \pm 1}$ we also know that $\mu_{1}=\mathcal{O}\left(h^{\infty}\right)$ (cf. $(11.13))$. Moreover, $-\Delta_{A}^{(1)}$ has precisely one eigenvalue $\widetilde{\mu}_{1}=o(h)$ and $-\Delta_{A}^{(k)}$ has no eigenvalues $=o(h)$ for $k \geq 2$. Since our Operators are real we know that the spectra are symmetric around the real axis, hence $\mu_{0}, \mu_{1}, \widetilde{\mu}_{1}$ are real. From the intertwining relations

$$
-\Delta_{A}^{(1)} d_{\phi}=d_{\phi}\left(-\Delta_{A}^{(0)}\right), \quad-\Delta_{A}^{(0)} d_{\phi}^{A, *}=-d_{\phi}^{A, *} \Delta_{A}^{(1)},
$$

we then also know that $\widetilde{\mu}_{1}=\mu_{1}$.

In fact, when $B>0$ it follows from the ellipticity and the estimates in Section 2 that all eigenforms and generalized eigenforms corresponding to an eigenvalue in $D(0, C h)$ belong to $\mathcal{S}\left(\mathbf{R}^{n}\right)$, so if $\mu_{1} \neq 0$ and $\left(-\Delta_{A}^{(0)}-\mu_{1}\right) u=0, u \in L^{2}$, then $u \in \mathcal{S}\left(\mathbf{R}^{n}\right)$ and $0 \neq d_{\phi} u \in \mathcal{S}$ is an eigenform for $-\Delta_{A}^{(1)}$ with the same eigenvalue. A priori we cannot exclude that $\mu_{1}=0$ and that $-\Delta_{A}^{(0)} u=$ Const $e^{-\phi / h}$. Then again, $0 \neq d_{\phi} u \in \mathcal{S}$ is a corresponding eigenvector of $-\Delta_{A}^{(1)}$. In the general case, we let $0<B_{\epsilon} \rightarrow B=: B_{0}$ when $\epsilon \searrow 0$. (Take for instance $B_{\epsilon}=B+\epsilon I$.) On a small circle $D(0, h / C)$ we know that $\left(P_{\epsilon}^{(q)}-z\right)^{-1}=\mathcal{O}\left(\frac{1}{h}\right)$ uniformly for $0 \leq \epsilon \ll 1$. If $\left(P_{0}^{(q)}-z\right) u=v, u, v \in \mathcal{S}$ (for $\left.\epsilon=0\right)$, then $\left(P_{\epsilon}^{(q)}-z\right) u=v+r_{\epsilon}$, $r_{\epsilon} \rightarrow 0$, so $\left(P_{\epsilon}^{(q)}-z\right)^{-1} v=u-\left(P_{\epsilon}^{(q)}-z\right)^{-1} r_{\epsilon} \rightarrow u, \epsilon \rightarrow 0$. Since $\left(P^{(q)}-z\right)(\mathcal{S})$ is dense in $L^{2}$ we conclude that $\left(P_{\epsilon}^{(q)}-z\right)^{-1} \rightarrow\left(P^{(q)}-z\right)^{-1}$ strongly for $|z|=h / C$. 
We have then the corresponding fact for the finite rank spectral projections and their compositions with $P_{\epsilon}^{(q)}$ :

$$
\frac{1}{2 \pi i} \int_{|z|=h / C}\left(z-P_{\epsilon}\right)^{-1} d z \text { and } \frac{1}{2 \pi i} \int_{|z|=h / C} z\left(z-P_{\epsilon}\right)^{-1} d z .
$$

These are finite rank Operators and converge therefore in norm. It follows that

$$
\sigma\left(P_{\epsilon}^{(q)}\right) \cap D(0, h / C) \rightarrow \sigma\left(P^{(q)}\right) \cap D(0, h / C), \quad \text { when } \quad \epsilon \rightarrow 0,
$$

so we get $\widetilde{\mu}_{1}=\mu_{1}$ also in the general case.

Let $\chi_{j} \in C_{0}^{\infty}\left(D_{j}\right)$ be equal to 1 on $\left.\left.D_{j} \cap \phi^{-1}(]-\infty, \phi\left(U_{0}\right)-\epsilon_{0}\right]\right)$ for $\epsilon_{0}>0$ fixed but arbitrarily small. Consider

$$
f_{j}=h^{-n / 4} c_{j}(h) e^{-\frac{1}{h}\left(\phi(x)-\phi\left(U_{j}\right)\right)} \chi_{j}(x), \quad j= \pm 1,
$$

where $c_{j} \sim c_{j, 0}+h c_{j, 1}+\cdots>0$ is a normalization constant with $c_{j, 0}>0$, such that

We also have

$$
\left\|f_{j}\right\|=1
$$

$$
P^{(0)}\left(f_{j}\right)=\left[P^{(0)}, \chi_{j}\right]\left(c_{j} e^{-\frac{1}{h}\left(\phi(x)-\phi\left(U_{j}\right)\right)}\right)=\mathcal{O}\left(h^{-N_{0}} e^{-\frac{1}{h}\left(S_{j}-\epsilon_{0}\right)}\right),
$$

for some $N_{0}>0$.

If

$$
\Pi^{(0)}=\frac{1}{2 \pi i} \int_{\gamma}\left(z-P^{(0)}\right)^{-1} d z, \quad \gamma=\partial D\left(0, \frac{h}{C}\right)
$$

is the spectral projection of $P^{(0)}$ onto $E^{(0)}$ we know from Theorem 8.4 that $\Pi^{(0)}=$ $\mathcal{O}(1)$. It follows from (11.11) that

$$
e_{j}:=\Pi^{(0)} f_{j}=f_{j}+\mathcal{O}\left(h^{-N_{1}} e^{-\frac{1}{h}\left(S_{j}-\epsilon_{0}\right)}\right) \quad \text { in } \quad L^{2} .
$$

In fact, we write $(11.11)$ as $P^{(0)} f_{j}=r_{j}$,

$$
\begin{aligned}
\left(z-P^{(0)}\right)\left(f_{j}\right) & =z f_{j}-r_{j}, \\
\left(z-P^{(0)}\right)^{-1} f_{j} & =\frac{1}{z} f_{j}+\left(z-P^{(0)}\right)^{-1} z^{-1} r_{j}
\end{aligned}
$$

and integrate, using the bounds on the resolvent provided by Theorem 8.4.

From (11.13) we see that

$$
\begin{aligned}
\left\|e_{j}\right\|^{2} & =1+\mathcal{O}\left(h^{-N_{2}} e^{-\frac{1}{h}\left(S_{j}-\epsilon_{0}\right)}\right), \\
\left(e_{1} \mid e_{-1}\right) & =\mathcal{O}\left(h^{-N_{2}} e^{-\frac{1}{h}\left(S_{\min }-\epsilon_{0}\right)}\right),
\end{aligned}
$$

where

$$
S_{\min }=\min \left(S_{-1}, S_{1}\right) \text {. }
$$

Let $E^{(1)}$ be the one-dimensional eigenspace of $P^{(1)}$ corresponding to $\mu_{1}$. From an easy extension of Theorem 9.1 to the non-scalar case with the presence of other non-resonant wells $\left(U_{ \pm 1}\right)$ as in Remark 9.2 , we know that $E^{(1)}$ is generated by an eigenform

$$
e_{0}(x ; h)=\chi_{0}(x) e^{-\frac{1}{h} \phi_{+}(x)} h^{-\frac{n}{4}} a_{0}(x ; h)+\mathcal{O}\left(e^{-S_{0} / h}\right),
$$


where $\chi_{0} \in C_{0}^{\infty}\left(\operatorname{neigh}\left(U_{0}\right)\right)$ is equal to one near $U_{0}, S_{0}>0$,

$$
a_{0}(x ; h) \sim \sum_{0}^{\infty} a_{0, k}(x) h^{k}
$$

is a symbol as in Theorem 9.1 with $a_{0,0}\left(U_{0}\right) \neq 0$, and $\phi_{+} \in C^{\infty}\left(\operatorname{neigh}\left(U_{0}\right) ;[0, \infty[)\right.$ satisfies

$$
\phi_{+}(x) \backsim\left|x-U_{0}\right|^{2}
$$

and solves the eikonal equation

$$
q\left(x, \phi_{+}^{\prime}(x)\right)=0
$$

with $q=p_{2}+p_{1}-p_{0}$,

$$
p_{2}=\langle B(x) \xi, \xi\rangle, \quad p_{1}(x, \xi)=2\left\langle C(x) \phi^{\prime}(x), \xi\right\rangle, \quad p_{0}(x)=\left\langle B(x) \phi^{\prime}(x), \phi^{\prime}(x)\right\rangle .
$$

$\Lambda_{\phi_{+}}$is the stable outgoing manifold through $\left(U_{0}, 0\right)$ for the $H_{q}$-flow and recall that $\phi_{+}^{\prime \prime}\left(U_{0}\right)>0$ by Proposition 8.2. (Similarly we have a stable incoming manifold $\Lambda_{\phi_{-}}$.) Let $k_{ \pm}$be the number of eigenvalues of the linearization of $H_{q_{\mid}}$ at that point with \pm real part $>0$, so that $k_{+}+k_{-}=n$. Let $K_{+}, K_{-} \subset \Lambda_{\phi}$ be the corresponding stable outgoing and incoming submanifolds of dimension $k_{+}$and $k_{-}$ respectively. Then $K_{+} \subset \Lambda_{\phi_{+}}, K_{-} \subset \Lambda_{\phi_{-}}$and $\phi-\phi\left(U_{0}\right)-\phi_{ \pm}$vanishes to the second order on $\pi_{x}\left(K_{ \pm}\right)$. Since $\phi^{\prime \prime}\left(U_{0}\right)$ has signature $(n-1,1)$, we conclude that $\operatorname{dim} K_{+}=n-1, \operatorname{dim} K_{-}=1$. (This also follows from Proposition 10.2.) It is also clear that $\Lambda_{\phi}, \Lambda_{\phi_{ \pm}}$intersect cleanly along $K_{ \pm}$, so we get

$$
\begin{aligned}
\phi_{+}-\left(\phi-\phi\left(U_{0}\right)\right) & \sim \operatorname{dist}\left(x, \pi_{x}\left(K_{+}\right)\right)^{2}, \\
\phi-\phi\left(U_{0}\right)-\phi_{-} & \sim \operatorname{dist}\left(x, \pi_{x}\left(K_{-}\right)\right)^{2} .
\end{aligned}
$$

We next make some remarks about the adjoint Operator $-\Delta_{t_{A}}=\left(-\Delta_{A}\right)^{t_{A}, *}$ (cf. Subsection 10.4). The principal symbol is $p_{2}-i p_{1}+p_{0}=p(x,-\xi)=\check{p}(x, \xi)$ and the corresponding real " $q$ "-symbol is $\check{q}(x, \xi)=q(x,-\xi)$. Since our dynamical conditions are invariant under a change of sign of the $H_{p_{1}}$-direction, all our assumptions are equally valid for $-\Delta_{t_{A}}$. This also holds for the geometric discussion above, so if $\Lambda_{\phi_{+}^{*}}, \Lambda_{\phi_{-}^{*}}$ denote the outgoing and incoming $H_{\breve{q}^{-}}$-invariant Lagrangian manifolds through $\left(U_{0}, 0\right)$ and $K_{ \pm}^{*} \subset \Lambda_{\phi}$ the outgoing/incoming manifolds for $H_{\left.\check{q}\right|_{\Lambda_{\phi}}}$ (noting that $\check{q}=0$ on $\Lambda_{\phi}$ ), then $\operatorname{dim} K_{+}^{*}=n-1, \operatorname{dim} K_{-}^{*}=1$ and

$$
\begin{aligned}
\phi_{+}^{*}-\left(\phi-\phi\left(U_{0}\right)\right) & \backsim \operatorname{dist}\left(x, \pi_{x}\left(K_{+}^{*}\right)\right)^{2}, \\
\phi-\phi\left(U_{0}\right)-\phi_{-}^{*} & \backsim \operatorname{dist}\left(x, \pi_{x}\left(K_{-}^{*}\right)\right)^{2} .
\end{aligned}
$$

In view of the general relation

$$
J_{*}\left(H_{q}\right)=-H_{\check{q}}, \quad \text { where } \quad J:(x, \xi) \mapsto(x,-\xi),
$$

we see that $\Lambda_{\phi_{-}^{*}}=J\left(\Lambda_{\phi_{+}}\right), \Lambda_{\phi_{+}^{*}}=J\left(\Lambda_{\phi_{-}}\right)$, or more simply

$$
\phi_{-}^{*}=-\phi_{+}, \quad \phi_{+}^{*}=-\phi_{-},
$$


giving in particular from (11.20), (11.21),

$$
\begin{aligned}
& \phi-\phi\left(U_{0}\right)+\phi_{+}^{*} \backsim \operatorname{dist}\left(x, \pi_{x}\left(K_{-}\right)\right)^{2}, \\
& \phi-\phi\left(U_{0}\right)+\phi_{+} \backsim \operatorname{dist}\left(x, \pi_{x}\left(K_{-}^{*}\right)\right)^{2} .
\end{aligned}
$$

Let $\mu_{0}^{*}=0, \mu_{1}^{*}$ be the two eigenvalues of $P_{*}^{(0)}:=-\Delta_{t_{A}}^{(0)}$ that are $o(h)$ and let $\Pi_{*}^{(0)}$ be the spectral projection onto the corresponding spectral subspace $E_{*}^{(0)}$. Then $e_{j}^{*}=\Pi_{*}^{(0)} f_{j}, j= \pm 1$, span $E_{*}^{(0)}$, and satisfy (11.13). Similarly for $P_{*}^{(1)}=$ $-\Delta_{t_{A}}^{(1)}$ we have the generating eigenform

$$
e_{0}^{*}(x ; h)=\chi_{0}(x) e^{-\frac{1}{h} \phi_{+}^{*}(x)} h^{-\frac{n}{4}} a_{0}^{*}(x ; h)+\mathcal{O}\left(e^{-S_{0} / h}\right) \text { in } L^{2}
$$

for the one dimensional eigenspace $E_{*}^{(1)}$ corresponding to $\mu_{1}^{*}$.

Now, using that our eigenvalues and Operators are real, we know by duality that

$$
\mu_{1}^{*}=\mu_{1},
$$

and that $\left(E_{*}^{(0)}, E^{(0)}\right)$ and $\left(E_{*}^{(1)}, E^{(1)}\right)$ are dual pairs for the scalar products $(u \mid v)_{L^{2}}$ and $(u \mid v)_{A}$ respectively. In fact, $\left(\left(z-P_{*}^{(q)}\right)^{-1}\right)^{A, *}=\left(\bar{z}-P^{(q)}\right)^{-1}$.

From Subsection 10.3 we know that $a_{0,0}\left(U_{0}\right)$ is an eigenvector corresponding to the negative eigenvalue of $\phi^{\prime \prime} \circ{ }^{t} A$ at $U_{0}$, and $a_{0,0}^{*}\left(U_{0}\right)$ is an eigenvector corresponding to the negative eigenvalue of $\phi^{\prime \prime} \circ A$. Since $\left(\phi^{\prime \prime} \circ A\right)^{A, *}=\phi^{\prime \prime} \circ{ }^{t} A$, we know that the two eigenvalues are equal and that the $A$-product of the two eigenvectors is $\neq 0$;

$$
\left(a_{0,0}^{*}\left(U_{0}\right) \mid a_{0,0}\left(U_{0}\right)\right)_{A} \neq 0 .
$$

It follows that $\left(e_{0}^{*} \mid e_{0}\right)_{A} \backsim 1$ and after renormalization of $e_{0}^{*}$ we may assume that

$$
\left(e_{0}^{*} \mid e_{0}\right)_{A}=1 .
$$

Similarly, using (11.10)

$$
\left(e_{j}^{*} \mid e_{k}\right)=\delta_{j, k}+\mathcal{O}\left(e^{-\frac{1}{C h}}\right), \quad j, k= \pm 1 .
$$

Let $\left(\begin{array}{ll}\lambda_{-1} & \lambda_{1}\end{array}\right)$ be the matrix of $d_{\phi}: E^{(0)} \rightarrow E^{(1)}$ with respect to the bases $\left(e_{-1}, e_{1}\right)$ and $\left(e_{0}\right)$. (Strictly speaking, we approximate our operators by elliptic ones as in (11.8) and pass to the limit.) Let

$$
\left(\begin{array}{c}
\lambda_{-1}^{*} \\
\lambda_{1}^{*}
\end{array}\right)
$$

be the matrix of $d_{\phi}^{A, *}$ for the same bases. The eigenvalue $\mu_{1}$ can be viewed as the second eigenvalue of $d_{\phi}^{A, *} d_{\phi}: E^{(0)} \rightarrow E^{(0)}$ or equivalently as the scalar $d_{\phi} d_{\phi}^{A, *}$ : $E^{(1)} \rightarrow E^{(1)}$ (using also that $P^{(2)}$ has no eigenvalue $=o(h)$ ). Either way, we get

$$
\mu_{1}=\lambda_{-1}^{*} \lambda_{-1}+\lambda_{1}^{*} \lambda_{1} .
$$


We get

$$
\begin{aligned}
& \bar{\lambda}_{k}=\left(e_{0}^{*} \mid d_{\phi} e_{k}\right)_{A}, \quad k= \pm 1, \\
& \bar{\lambda}_{j}^{*}=\left(g_{j} \mid d_{\phi}^{A, *} e_{0}\right)_{A}, \quad j= \pm 1,
\end{aligned}
$$

where

$$
\left(\begin{array}{ll}
g_{-1} & g_{1}
\end{array}\right)=\left(\begin{array}{ll}
e_{-1}^{*} & e_{1}^{*}
\end{array}\right)\left(1+\mathcal{O}\left(e^{-\frac{1}{C h}}\right)\right)
$$

is the base in $E_{*}^{(0)}$ that is dual to $\left(\begin{array}{ll}e_{-1} & e_{1}\end{array}\right)$. Here the complex conjugate signs are superfluous since we work with real Operators, eigenvalues and functions.

Let $\chi \in C_{0}^{\infty}\left(\operatorname{neigh}\left(U_{0}\right) ;[0,1]\right)$ be equal to 1 near $U_{0}$. Using that $d_{\phi} e_{-1}=$ $\lambda_{-1} e_{0}$, we get, dropping the bars from now on,

$$
\begin{aligned}
\lambda_{-1} & =\left(e_{0}^{*} \mid d_{\phi} e_{-1}\right)_{A} \\
& =\left(e_{0}^{*} \mid \chi d_{\phi} e_{-1}\right)_{A}+\lambda_{-1}\left(e_{0}^{*} \mid(1-\chi) e_{0}\right)_{A} \\
& =\left(e_{0}^{*} \mid \chi d_{\phi} e_{-1}\right)_{A}+\mathcal{O}\left(e^{-\frac{1}{C h}}\right) \lambda_{-1} .
\end{aligned}
$$

Here

$$
\left(e_{0}^{*} \mid \chi d_{\phi} e_{-1}\right)_{A}=\left(e_{0}^{*} \mid\left[\chi, d_{\phi}\right] e_{-1}\right)_{A}+\left(d_{\phi}^{t} A, * * e_{0}^{*} \mid \chi e_{-1}\right)_{A} .
$$

Now the matrix of $d_{\phi}^{t A, *}: E_{*}^{(1)} \rightarrow E_{*}^{(0)}$ with respect to the dual bases is the adjoint of the one of $d_{\phi}: E^{(0)} \rightarrow E^{(1)}$, so $d_{\phi}^{t} A, * e_{0}^{*}=\lambda_{-1} g_{-1}+\lambda_{1} g_{1}$, and expressing $g_{j}$ as linear combinations of the $e_{j}^{*}$ by means of (11.33) and using (11.13) for the $e_{ \pm 1}^{*}$ we see that the last term in (11.24) is of the form

$$
\left(d_{\phi}^{t} A, * e_{0}^{*} \mid \chi e_{-1}\right)_{A}=\mathcal{O}\left(e^{-\frac{1}{C h}}\right) \lambda_{-1}+\mathcal{O}\left(e^{-\frac{1}{C h}}\right) \lambda_{1} .
$$

Thus we have obtained

$$
\begin{aligned}
\left(1+\mathcal{O}\left(e^{-\frac{1}{C h}}\right)\right) \lambda_{-1}+\mathcal{O}\left(e^{-\frac{1}{C h}}\right) \lambda_{1} & =\left(e_{0}^{*} \mid\left[\chi, d_{\phi}\right] e_{-1}\right)_{A} \\
& =-h\left(e_{0}^{*} \mid(d \chi) \wedge e_{-1}\right)_{A},
\end{aligned}
$$

and we shall study the last expression. The contribution from the remainder in (11.13) is $\mathcal{O}\left(h^{-N_{2}}\right) \exp \frac{1}{h}\left(-S_{-1}+\epsilon_{0}-\frac{1}{C}\right)=\mathcal{O}(1) e^{-\frac{1}{h}\left(S_{-1}+\frac{1}{2 C}\right)}$ if we choose $\epsilon_{0}$ small enough. A similar estimate holds for the contribution from the remainder term in (11.25). As we shall see, the contribution from the leading terms in (11.13), (11.25) will be larger. It is equal to

$$
-c_{-1}(h) h^{1-\frac{n}{2}} \int \chi_{-1}(x)\left\langle A(x) a_{0}^{*}(x ; h) \mid d \chi(x)\right\rangle e^{-\frac{1}{h}\left(\phi_{+}^{*}(x)+\phi(x)-\phi\left(U_{-1}\right)\right)} d x .
$$

Here by (11.24),

$$
\begin{aligned}
\phi_{+}^{*}(x)+\phi(x)-\phi\left(U_{-1}\right)=\phi_{+}^{*}+\left(\phi(x)-\phi\left(U_{0}\right)\right) & +S_{-1} \\
& \sim S_{-1}+\operatorname{dist}\left(x, \pi_{x}\left(K_{-}\right)\right)^{2},
\end{aligned}
$$

so we expect (11.38) to behave like some power of $h$ times $e^{-\frac{1}{h} S_{-1}}$ with the main contribution coming from a neighborhood of $\operatorname{supp}(d \chi) \cap \operatorname{supp}\left(\chi_{-1}\right) \cap \pi_{x}\left(K_{-}\right)$. Now $\phi(x)-\phi\left(U_{0}\right) \backsim-\left|x-U_{0}\right|^{2}$ on $\pi_{x}\left(K_{-}\right)$while $\chi_{-1}$ has its support in $D_{-1}$ and 
equals 1 in the subset of $D_{-1}$ where $\phi(x)-\phi\left(U_{0}\right) \leq-\epsilon_{0}$ with $\epsilon_{0}$ arbitrarily small. Because of the presence of $d \chi$ which has its support in an annular region around $U_{0}$, we see that $\chi_{-1}(x)=1$ in neigh $\left(D_{-1} \cap \operatorname{supp}(d \chi) \cap \pi_{x}\left(K_{-}\right)\right)$, so we can forget about $\chi_{-1}$ in (11.38) and just integrate over neigh $\left(\operatorname{supp}(d \chi) \cap \pi_{x}\left(K_{-1}\right), D_{-1}\right)$.

Let us look at $\left\langle A(x) a_{0,0}^{*}(x ; h) \mid d \chi\left(x_{1}\right)\right\rangle$ at a point $x_{1} \in \operatorname{supp}(d \chi) \cap \pi_{x}\left(K_{-}\right)$. At $U_{0}$ we know that $a_{0,0}^{*}$ is an eigenvector of $\phi^{\prime \prime} \circ A$ associated to the negative eigenvalue, so $A a_{0,0}^{*}$ is a corresponding eigenvector for $A \phi^{\prime \prime}$ which is the linearization of $\frac{1}{2} H_{q_{\Lambda_{\phi}}}$. Hence $A a_{0,0}^{*}$ at $U_{0}$ is tangent to $\pi_{x}\left(K_{-}\right)$. This will remain approximately true at $x_{1}$ since the latter point is close to $U_{0}$. Choosing $\chi$ to be a "circular" standard cut-off, we see that $\left\langle A a_{0,0}^{*}, d \chi\right\rangle$ is non-vanishing of constant sign at every point $x_{1} \in \pi_{x}\left(K_{-}\right) \cap D_{-1}$ where $d \chi \neq 0$.

By stationary phase it is now clear that the integral (11.38) is equal to

$$
h^{\frac{1}{2}} \ell_{-1}(h) e^{-\frac{1}{h} S_{-1}}, \quad \ell_{-1} \sim \ell_{-1,0}+h \ell_{-1,1}+\cdots, \quad \ell_{-1,0} \neq 0 .
$$

Returning to (11.37) and modifying $\ell_{-1}$ by an exponentially small term, we get

$$
\left(1+\mathcal{O}\left(e^{-\frac{1}{C h}}\right)\right) \lambda_{-1}+\mathcal{O}\left(e^{-\frac{1}{C h}}\right) \lambda_{1}=h^{\frac{1}{2}} \ell_{-1}(h) e^{-\frac{1}{h} S_{-1}} .
$$

Similarly,

$$
\begin{aligned}
\mathcal{O}\left(e^{-\frac{1}{C h}}\right) \lambda_{-1}+\left(1+\mathcal{O}\left(e^{-\frac{1}{C h}}\right)\right) \lambda_{1}= & h^{\frac{1}{2}} \ell_{1}(h) e^{-\frac{1}{h} S_{1}}, \\
& \ell_{1} \sim \ell_{1,0}+h \ell_{1,1}+\cdots, \quad \ell_{1,0} \neq 0 .
\end{aligned}
$$

Inverting the system, we get

$$
\left(\begin{array}{c}
\lambda_{-1} \\
\lambda_{1}
\end{array}\right)=\left(1+\mathcal{O}\left(e^{-\frac{1}{C h}}\right)\right)\left(\begin{array}{c}
h^{\frac{1}{2}} \ell_{-1}(h) e^{-\frac{1}{h} S_{-1}} \\
h^{\frac{1}{2}} \ell_{1}(h) e^{-\frac{1}{h} S_{1}}
\end{array}\right) .
$$

Now turn to $\lambda_{j}^{*}$ in (11.32). In view of (11.33), we have

$$
\left(\begin{array}{c}
\lambda_{-1}^{*} \\
\lambda_{1}^{*}
\end{array}\right)=\left(1+\mathcal{O}\left(e^{-\frac{1}{C h}}\right)\right)\left(\begin{array}{c}
\alpha_{-1} \\
\alpha_{1}
\end{array}\right),
$$

where

$$
\alpha_{j}=\left(d_{\phi} e_{j}^{*} \mid e_{0}\right)_{A}=\left(e_{0} \mid d_{\phi} e_{j}^{*}\right)^{t_{A}}
$$

which can be identified with the expression (11.31) after replacing $A$ by ${ }^{t} A$ and making the corresponding substitutions, $e_{0}^{*} \rightarrow e_{0}, e_{j} \rightarrow e_{j}^{*}$. Hence we have the analogue of (11.43),

$$
\begin{gathered}
\left(\begin{array}{c}
\lambda_{-1}^{*} \\
\lambda_{1}^{*}
\end{array}\right)=\left(1+\mathcal{O}\left(e^{-\frac{1}{C h}}\right)\right)\left(\begin{array}{c}
\alpha_{-1} \\
\alpha_{1}
\end{array}\right)=\left(1+\mathcal{O}\left(e^{-\frac{1}{C h}}\right)\right)\left(\begin{array}{c}
h^{\frac{1}{2}} \ell_{-1}^{*}(h) e^{-\frac{1}{h} S_{-1}} \\
h^{\frac{1}{2}} \ell_{1}^{*}(h) e^{-\frac{1}{h} S_{1}}
\end{array}\right), \\
\ell_{j}^{*}(h) \sim \ell_{j, 0}^{*}+h \ell_{j, 1}^{*}+\cdots, \quad \ell_{j, 0}^{*} \neq 0 .
\end{gathered}
$$

We finally claim that $\ell_{j, 0} \ell_{j, 0}^{*}>0$. Indeed, this number is real and different from zero and if we deform our matrices to reach the selfadjoint case (with $A>0$ ) we see that we have a positive sign.

Combining this with (11.30) we get the main result of this work: 
Theorem 11.1. Let $P=-\Delta_{A}^{(0)}$ where we assume (11.1), (11.2), (11.7). We also assume that $P$ satisfies the additional dynamical conditions (4.21), (4.22) (or equivalently (4.21), (4.24)) and (4.23). Then for $C>0$ large enough, $P$ has precisely 2 eigenvalues, 0 and $\mu_{1}$ in the disc $D(0, h / C)$ when $h>0$ is small enough. Here $\mu_{1}$ is real and of the form

$$
\mu_{1}=h\left(a_{1}(h) e^{-2 S_{1} / h}+a_{-1}(h) e^{-2 S_{-1} / h}\right),
$$

where $a_{j}(h)$ are real, $a_{j}(h) \sim a_{j, 0}+a_{j, 1} h+\cdots, a_{j, 0}>0, S_{j}=\phi\left(U_{0}\right)-\phi\left(U_{j}\right)$.

\section{Acknowledgements}

We are grateful to B. Helffer who pointed out the work [24] to us, and for pointing out that an earlier version of our condition (11.2) was not directly applicable to the Kramers-Fokker-Planck operator. The research of the second author is supported in part by the National Science Foundation under grant DMS-0304970 and by an Alfred P. Sloan Research Fellowship. He is happy to acknowledge the hospitality of École Polytechnique and Université de Reims, where part of this work was done.

\section{References}

[1] D. Bao, S.-S. Chern, Z. Shen, An introduction to Riemann-Finsler geometry, Graduate texts in Mathematics, 200. Springer-Verlag, New York, 2000.

[2] J. M. Bismut, The hypoelliptic Laplacian on the cotangent bundle, J. Amer. Math. Soc. 18 (2005), 379-476.

[3] J. M. Bismut, G. Lebeau, The hypoelliptic Laplacian and Ray-Singer metrics, preprint (2006).

[4] L. Desvillettes and C. Villani, On the trend to global equilibrium in spatially inhomogeneous entropy-dissipating systems: the linear Fokker-Planck equation, Comm. Pure Appl. Math., 54 (1) (2001), 1-42.

[5] J.-P. Eckmann, M. Hairer, Spectral properties of hypoelliptic operators, Comm. Math. Phys. 235 (2) (2003), 233-253.

[6] M. I. Freidlin, A. D. Wentzell, Random perturbations of dynamical systems, SpringerVerlag, New York, 1984.

[7] B. Helffer, M. Klein, F. Nier, Quantitative analysis of metastability in reversible diffusion processes via a Witten complex approach, Mat. Contemp. 26 (2004), 41-85.

[8] B. Helffer, F. Nier, Hypoelliptic estimates and spectral theory for Fokker-Planck operators and Witten Laplacians, Lecture Notes in Mathematics, 1862. Springer-Verlag, New York, 2005.

[9] B. Helffer, J. Sjöstrand, Multiple wells in the semiclassical limit. I, Comm. Partial Differential Equations 9 (4) (1984), 337-408, Puits multiples en limite semi-classique. II. Interaction moléculaire. Symétries. Perturbation, Ann. Inst. H. Poincaré Phys. Théor. 42 (2) (1985), 127-212.

[10] B. Helffer, J. Sjöstrand, Multiple wells in the semiclassical limit. III. Interaction through nonresonant wells, Math. Nachr. 124 (1985), 263-313. 
[11] B. Helffer, J. Sjöstrand, Puits multiples en mécanique semi-classique. IV. Etude du complexe de Witten, Comm. Partial Differential Equations 10 (3) (1985), 245-340.

[12] B. Helffer, J. Sjöstrand, Résonances en limite semi-classique, Bull. de la S.M.F., Mémoire 24/25, Suppl. du Tome 114 (3) (1986).

[13] F. Hérau, F. Nier, Isotropic hypoellipticity and trend to equilibrium for the FokkerPlanck equation with a high-degree potential, Arch. Ration. Mech. Anal. 171 (2) (2004), 151-218.

[14] F. Hérau, J. Sjöstrand, C. Stolk, Semiclassical analysis for the Kramers-FokkerPlanck equation, Comm. Partial Differential Equations 30 (4-6) (2005), 689-760.

[15] M. Hitrik, Boundary spectral behavior for semiclassical operators in dimension one, Int. Math. Res. Not. 64 (2004), 3417-3438.

[16] V. N. Kolokoltsov, Semiclassical analysis for diffusions and stochastic processes, Lecture Notes in Mathematics, 1724, Springer-Verlag, Berlin, 2000.

[17] G. Lebeau, Le bismutien, Séminaire équations aux dérivées partielles, Ecole Polytechnique 2004-05, I.1-I.15.

[18] Y. Li, L. Nirenberg, The distance function to the boundary, Finsler's geometry and the singular set of viscosity solutions of some Hamilton-Jacobi equations, Comm. Pure Appl. Math, 58 (2005), 85-146.

[19] A. Melin, J. Sjöstrand, Fourier integral operators with complex-valued phase functions, Springer Lect. Notes in Math., 459.

[20] A. Melin, J. Sjöstrand, Determinants of pseudodifferential operators and complex deformations of phase space, Meth. Appl. Analysis 9 (2002), 177-238.

[21] J. Sjöstrand, M. Dimassi, Spectral asymptotics in the semi-classical limit, London Math. Soc. Lecture Notes Series 269, Cambridge University Press 1999.

[22] J. Sjöstrand, Parametrices for pseudodifferential operators with multiple characteristics, Ark. f. Mat. 12 (1) (1974), 85-130.

[23] J. Sjöstrand, Density of resonances for strictly convex obstacles, Can. J. Math. 48 (2) (1996), 397-447.

[24] J. Tailleur, S. Tanase-Nicola, J. Kurchan, Kramers equation and supersymmetry, J. Stat. Phys. 122 (4) (2006), 557-595. (preprint: arxiv.org/abs/cond-mat/0503545).

[25] C. Villani, Hypocoercivity, preprint, 2006 (arxiv.org/abs/math.AP/0609050).

Frédéric Hérau

Laboratoire de Mathématiques

UMR 6056-CNRS

Université de Reims

Moulin de la Housse

B.P. 1039

F-51687 Reims Cedex 2

France

e-mail: herau@univ-reims.fr 
Michael Hitrik

Department of Mathematics

University of California

Los Angeles, CA 90095-1555

USA

e-mail: hitrik@math.ucla.edu

Johannes Sjöstrand

CMLS

UMR7640-CNRS

Ecole Polytechnique

F-91120 Palaiseau Cedex

France

e-mail: johannes@math.polytechnique.fr

Communicated by Christian Gérard.

Submitted: April 3, 2007.

Accepted: October 4, 2007. 